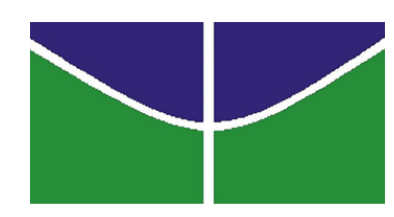

Universidade de Brasília Instituto de Ciências Exatas

Departamento de Matemática

\title{
Comportamento Assintótico de Cadeias de Markov via Distância Mallows, com Aplicação em Processos Empíricos
}

\author{
por
}

Edimilson dos Santos da Silva

Brasília, Novembro de 2016. 


\section{Agradecimentos}

Neste momento especial em minha vida, que coroa uma etapa de muitas batalhas e lutas, agradeço primeiramente a Deus, por ter me dado forças para enfrentar todos os obstáculos e a fé necessária para sempre acreditar em meu objetivo.

Agradeço à banca examinadora pelas observações e críticas construtivas que em muito ajudaram a melhorar o texto final desta tese. Em especial, sou muito grato à professora Cátia por sua gentileza em sempre se dispor a me ajudar a esclarecer dúvidas importantes, e por ser um exemplo de professora no qual sempre me espelharei.

À minha orientadora, professora Chang Dorea, não tenho palavras que possam descrever a eterna gratidão que sempre terei. Sinto-me muito orgulhoso por ter tido a oportunidade de ser orientado por uma profissional de tamanha competência e, acima de tudo, uma pessoa extremamente sensível e generosa. Muito obrigado, professora Chang!

Ficam aqui também meus agradecimentos aos professores do Departamento de Matemática da UnB com os quais tive aula, aos funcionários da instituição e colegas de curso que comigo compartilharam momentos importantes nesta caminhada.

Ao concretizar esta importante etapa de minha carreira acadêmica, serei eternamente grato a todos que colaboraram para a realização deste sonho, em especial à minha sempre professora Roseane Cristina, aos professores Douglas e Nelson, e à generosidade de Dona Sandra e família.

À luz das emoções, parabenizo meus pais, Nilson e Jucileide, e minha avó Júlia Maria, pela vitória obtida por este filho e neto que muito os ama. Amor este que também sempre sentirei pela minha esposa Leidiane, companheira de todas as horas e a quem também especialmente dedico esta conquista.

Obrigado, também, à Capes e ao $\mathrm{CNPq}$ pelo auxílio financeiro concedido.

Agradeço a todos e, acima de tudo, ao maior dos matemáticos: Deus! 


\section{Resumo}

Nesta tese estudamos o comportamento assintótico de somas parciais de variáveis aleatórias que constituem uma cadeia de Markov $X=\left\{X_{n}\right\}_{n \geq 0}$. Assim, provamos a convergência, em distância Mallows, de somas parciais associadas a cadeias de Markov com espaço de estados enumerável para uma variável aleatória $\alpha$-estável, com $1<\alpha \leq 2$, abordando, separadamente, o caso Gaussiano e o caso cauda-pesada. Como uma aplicação, demonstramos a convergência fraca de um tipo especial de soma parcial, o processo empírico $\beta_{n}(x)$ relativo a uma cadeia de Markov com espaço de estados geral, bem como do processo considerado o seu inverso, o processo quantil empírico $q_{n}(t)$.

Palavras-chave: cadeias de Markov, distribuições estáveis, distância Mallows, processos empíricos. 


\section{Abstract}

In this dissertation we prove the convergence in Mallows distance of partial sums of random variables associated with a Markov chain with countable state space to a $\alpha$-stable random variable, with $1<\alpha \leq 2$, addressing separately the Gaussian case and the heavy-tailed case. As an application, we prove the weak convergence of a special type of partial sum, the empirical process $\beta_{n}(x)$ on a Markov chain with general state space, as well as its inverse process, the empirical quantile process $q_{n}(t)$.

Keywords: Markov chains, stable distributions, Mallows distance, empirical processes. 


\section{Sumário}

$\begin{array}{ll}\text { Introdução } & 1\end{array}$

1 Conceitos Preliminares $\quad 4$

1.1 Notações e Abreviações . . . . . . . . . . . . . . . . . . . . . . . . . 4

1.2 Cadeias de Markov . . . . . . . . . . . . . . . . . . . . 5

1.3 Distribuições Estáveis . . . . . . . . . . . . . . . . . . . 7

1.4 Distância Mallows . . . . . . . . . . . . . . . . . . . 8

1.5 Resultados Relacionados . . . . . . . . . . . . . . . . . . . . . 10

1.6 Processos Empíricos . . . . . . . . . . . . . . . . . . . . 12

1.7 Sobre Convergência Fraca . . . . . . . . . . . . . . . . 15

2 Comportamento Assintótico de Cadeias de Markov via Distância Mallows 19

2.1 Introdução . . . . . . . . . . . . . . . . . . . . . . . . . . 19

2.2 Resultados Preliminares . . . . . . . . . . . . . . . . . . . 20

2.3 O Caso Gaussiano . . . . . . . . . . . . . . . . . . . . . . . 28

2.4 O Caso Cauda-Pesada . . . . . . . . . . . . . . . . . . . . . . 39

3 Convergência de Processos Empíricos Associados a Cadeias de Markov com Espaço de Estados Geral

3.1 Introdução . . . . . . . . . . . . . . . . . . . . 54

3.2 Convergênca Fraca do Processo Empírico . . . . . . . . . . . . . . . . 55

3.2.1 Convergência no Sentido das Distribuições Finitas . . . . . . . . . . . 56

3.2.2 A Rigidez de $\left\{\beta_{n}\right\} \ldots \ldots \ldots \ldots \ldots \ldots$

3.3 Convergência Fraca do Processo Quantil Empírico $q_{n}(t) \ldots \ldots \ldots$. . . . . . . . 61

3.3.1 Convergência de $q_{n}(t)$ para $t$ fixado . . . . . . . . . . . . . 61

3.3.2 Convergência de $q_{n}(t)$ como Processo . . . . . . . . . . . 63

$\begin{array}{ll}\text { Bibliografia } & 65\end{array}$ 


\section{Introdução}

Nesta tese, nosso tema central é o estudo do comportamento assintótico de somas parciais de variáveis aleatórias que constituem uma cadeia de Markov $X=\left\{X_{n}\right\}_{n \geq 0}$, através de ferramentas como a distância Mallows. Como uma aplicação direta, estabeleceremos a convergência fraca de um tipo especial de soma parcial, o processo empírico $\beta_{n}(x)$ associado a uma cadeia de Markov com espaço de estados geral, bem como de seu processo inverso, o processo quantil empírico $q_{n}(t)$.

Assim, inicialmente provaremos a convergência, em distância Mallows, de uma soma parcial de variáveis aleatórias que constituem uma cadeia de Markov com espaço de estados enumerável, para uma variável aleatória $\alpha$-estável, com $1<\alpha \leq 2$, abordando, separadamente, o caso gaussiano e o caso cauda-pesada.

A propósito, a distância Mallows de ordem $r>0$ entre duas funções de distribuição $F$ e $G$ é dada pela expressão

$$
d_{r}(F, G)=\left(\inf _{(X, Y)} E|X-Y|^{r}\right)^{\frac{1}{r}},
$$

onde o ínfimo é tomado sobre todos os vetores aleatórios $(X, Y)$ com distribuições marginais $F$ e $G$, ou seja, $X$ tem distribuição $F$, e $Y$ tem distribuição $G$. Além disso, essa expressão é uma métrica no espaço das distribuições com $r$-ésimo momento finito, descrito por

$$
\mathcal{L}^{r}=\left\{F \text { é função de distribuição; } \int_{-\infty}^{\infty}|x|^{r} d F(x)<\infty\right\} .
$$

Essa poderosa ferramenta tem sido sucessivamente usada para obter teoremas do tipo limite central para distribuições $\alpha$-estáveis. Citamos, por exemplo, os resultados de Johnson e Samworth (2005) para o caso gaussiano, e os resultados do tipo Lindeberg em Barbosa e Dorea (2009) para o caso nãogaussiano. Além disso, a opção por essa técnica pode ser justificada por trabalhos como o de Bickel e Freedman (1981), que mostraram, entre outros detalhes, que a convergência nesta métrica, para $r \geq 1$, implica a convergência tanto em distribuição como dos momentos associados.

Provados os resultados para somas parciais de variáveis aleatórias constituindo uma cadeia de Markov enumerável, o próximo passo lógico é provar a convergência fraca para um tipo particular de soma parcial: o processo empírico. Assim, dadas variáveis aleatórias $X_{1}, X_{2}, \ldots, X_{n}$ com função de distribuição $F$, o processo empírico associado a essas variáveis aleatórias é definido como

$$
\beta_{n}(x)=\sqrt{n}\left(F_{n}(x)-F(x)\right), x \in \mathbb{R}, \quad n \geq 1,
$$


em que

$$
F_{n}(x, \omega)=\frac{\sum_{i=1}^{n} \mathbb{I}_{\left(X_{i}(\omega) \leq x\right)}}{n}
$$

é a função de distribuição empírica relativa a essas mesmas variáveis aleatórias.

Além disso, tendo em vista a convergência desejada, um processo estocástico $\{B(t), 0 \leq t \leq 1\}$ é denominado Ponte Browniana se

$$
B(t)=\tilde{W}(t)-t \tilde{W}(1), 0 \leq t \leq 1,
$$

em que $\{\tilde{W}(t), 0 \leq t<\infty\}$ é um Movimento Browniano.

No caso i.i.d., conforme Billingsley (1968), é fato conhecido a convergência fraca de $\beta_{n}(x)$ para $B(F(x))$, para $0<F(x)<1$. À luz desse resultado clássico, nós provamos, para uma cadeia de Markov com espaço de estados geral e ergódica, que essa mesma convergência fraca vale. Para tal propósito, novamente foi fundamental a distância Mallows, agora para obtermos a convergência das distribuições finitas.

Após descrevermos a importância da distância Mallows e nossos resultados relacionados a somas parciais, especificamente o processo empírico, tratamos, agora, do resultado obtido para o processo considerado o inverso desse último, o qual denominamos processo quantil empírico.

Dadas variáveis aleatórias $X_{1}, X_{2}, \ldots, X_{n}$ com função de distribuição $F$, definimos o processo quantil empírico associado a essas variáveis aleatórias, como

$$
q_{n}(t)=\sqrt{n}\left(F_{n}^{-}(t)-F^{-}(t)\right), t \in(0,1), \quad n \geq 1
$$

em que

$$
F_{n}^{-}(t, \omega)=\inf \left\{x ; F_{n}(x, \omega) \geq t\right\}
$$

é a inversa generalizada empírica ou função quantil empírica, e

$$
F^{-}(t)=\inf \{x ; F(x) \geq t\}, \quad F^{-}(0)=F^{-}\left(0^{+}\right)
$$

é a inversa generalizada de $F$.

Sabe-se que há uma dificuldade intrínseca na tentativa de obter a convergência fraca do processo quantil empírico diretamente, com a mesma metodologia aplicada para o caso empírico. Assim, a estratégia que adotamos em nosso trabalho é usar algum método alternativo, a fim de eliminarmos essa dificuldade e obtermos a convergência desejada. Para isso, a partir do caso empírico, usamos o Lema de Vervaat e o Teorema de Skorohod, bem como as versões uniformes dos processos empírico e quantil empírico, para obtermos a convergência fraca de $q_{n}(t)$. Essa convergência já é conhecida para o caso i.i.d., conforme Csörgo e Révész (1981). Em nosso caso, nós a obtemos para variáveis aleatórias que constituem uma cadeia de Markov com espaço de estados geral e ergódica.

Após essa breve abordagem de nossos objetivos, motivações e ferramentas utilizadas, descrevemos, agora, como está organizada esta tese. Uma melhor caracterização de cada capítulo será fornecida na introdução de cada um deles. 
No Capítulo 1, apresentamos conceitos e resultados preliminares que são fundamentais para o bom entendimento dos capítulos seguintes. Assim, abordamos aspectos teóricos relativos a cadeias de Markov, distribuições estáveis, distância Mallows, processos empíricos, convergência fraca e rigidez, bem como resultados relacionados sobre integrabilidade uniforme e desigualdades para martingales.

No Capítulo 2, provamos a convergência, em distância Mallows, de uma soma parcial de variáveis aleatórias que constituem uma cadeia de Markov com espaço de estados enumerável, para uma variável aleatória $\alpha$-estável, com $1<\alpha \leq 2$, abordando, separadamente, o caso gaussiano e o caso caudapesada. O caso gaussiano, por sua vez, conecta-se com os resultados obtidos no Capítulo 3 relativos ao processo empírico, que é um tipo de soma parcial de variáveis aleatórias.

No Capítulo 3, demonstramos a convergência fraca, sob condições adequadas, dos processos empírico e quantil empírico, associados a uma cadeia de Markov com espaço de estados geral, ergódica. Assim, na Subseção 3.2.1 provamos a convergência, através da distância Mallows, das respectivas distribuições finitas do processo empírico $\beta_{n}(t)$ e de $B(t)$ e, na Subseção 3.2.2, provamos a rigidez de $\left\{\beta_{n}\right\}$. Por fim, na Seção 3.3, usamos a convergência fraca do processo empírico obtida na Seção 3.2, para provarmos a convergência fraca do processo quantil empírico $q_{n}(t)$ associado a uma cadeia de Markov ergódica com espaço de estados geral, sob condições adequadas. Assim, na Subseção 3.3.1 provamos essa convergência para $t$ fixado, visando a uma melhor compreensão da seção seguinte por parte do leitor deste texto e, na Subseção 3.3.2, provamos a convergência fraca desejada de $q_{n}(t)$, para todo $t$ em $(0,1)$. 


\section{Capítulo 1}

\section{Conceitos Preliminares}

\subsection{Notações e Abreviações}

$\stackrel{d}{\longrightarrow}$ : Convergência em distribuição;

$\stackrel{d}{=}$ : Igualdade em distribuição;

$\stackrel{p}{\longrightarrow}$ : Convergência em probabilidade;

$\stackrel{\text { q.c. }}{\longrightarrow}$ : Convergência quase-certa;

$\stackrel{d_{\alpha}}{\longrightarrow}$ : Convergência na $\alpha$-ésima distância Mallows;

$\|\cdot\|_{p}:$ Norma em $L^{p}$

$\stackrel{\text { l.u. }}{\longrightarrow}$ : Convergência localmente uniforme;

i.i.d. : Independentes e identicamente distribuídas;

$\Rightarrow$ : Convergência fraca;

$\sigma(X): \sigma$-álgebra gerada por $X$;

$a \wedge b: \operatorname{Min}\{\mathrm{a}, \mathrm{b}\}$

$\mathcal{N}\left(\mu, \sigma^{2}\right)$ : Distribuição normal com média $\mu$ e variância $\sigma^{2}$;

$C[0,1]$ : Espaço das funções $g:[0,1] \rightarrow \mathbb{R}$ contínuas;

$D[0,1]$ : Espaço das funções $h:[0,1] \rightarrow \mathbb{R}$ contínuas pela direita e com limite finito pela esquerda. 


\subsection{Cadeias de Markov}

Nesta seção, abordamos inicialmente a teoria básica relativa a cadeias de Markov com espaço de estados enumerável e, posteriormente, para espaço de estados geral. Assim, consideraremos, a seguir, cadeias de Markov $X=\left\{X_{n}\right\}_{n \geq 0}$ com espaço de estados enumerável $S$ e matriz de transição $P=\left(P_{i j}\right)$.

Note que se a cadeia $X$ é ergódica, então

$$
\lim _{n \rightarrow \infty} P_{i j}^{n}=\pi_{j}, \forall i \in S, \forall j \in S
$$

onde $P_{i j}{ }^{n}=P\left(X_{n}=j / X_{0}=i\right)$. Neste caso, $\pi=\left(\pi_{j}\right)_{j \in S}$ é a única distribuição que é distribuição limite. Também, para cadeias ergódicas existe no máximo um subconjunto fechado de estados recorrentes e aperiódicos. Pela teoria clássica de cadeias de Markov (veja, por exemplo, Çinlar (1975) ou Breiman (1992)) temos para o tempo da k-ésima visita ao estado $i$,

$$
0=\tau_{0}(i)<\tau_{1}(i)<\tau_{2}(i)<\ldots<\tau_{k}(i)<\ldots,
$$

onde $\tau_{k}(i)=\inf \left\{n ; n>\tau_{k-1}(i), X_{n}=i\right\}, k \geq 1$.

Nos resultados abaixo a seguinte notação é usada: se $\nu$ é uma distribuição inicial, nós denotamos sua correspondente probabilidade e esperança por $P_{\nu}$ e $E_{\nu}$, respectivamente; e se $\nu$ está concentrada no estado $i$ nós simplesmente escrevemos $P_{i}$ e $E_{i}$.

Proposição 1.1 Seja X uma cadeia de Markov aperiódica, recorrente positiva e, para $i \in S$, seja $\left\{\tau_{k}(i)\right\}_{k \geq 0}$ definido por (1.2). Então (1.1) vale e

a) As variáveis $\left\{\tau_{k}(i)\right\}_{k \geq 0}$ são tempos de parada com respeito às $\sigma$-álgebras $\left\{\sigma\left(X_{0}, \ldots, X_{k}\right)\right\}_{k \geq 0}$ $e$, para $k \geq 1,\left\{\tau_{k+1}(i)-\tau_{k}(i)\right\}$ são independentes e identicamente distribuídas com tempo médio de recorrência dado por

$$
E\left\{\tau_{k+1}(i)-\tau_{k}(i)\right\}=m_{i}=\frac{1}{\pi_{i}} .
$$

Se $\pi_{i}=0$ nós consideramos $m_{i}=\infty$.

b) O processo $X_{\tau_{k}(i)+1}, X_{\tau_{k}(i)+2}, \ldots$, tem a mesma distribuição que $X_{1}, X_{2}, \ldots$, e é independente de $\sigma\left(X_{n}, n \leq \tau_{k}(i)\right)$.

c) Se $V_{k}$ é $\sigma\left(X_{\tau_{k}(i)}, X_{\tau_{k}(i)+1}, \ldots, X_{\tau_{k+1}(i)-1}\right)$-mensurável, então $V_{0}, V_{1}, V_{2}, \ldots$, são independentes e identicamente distribuídas sob $P_{i}$.

A seguir, nós estabelemos uma proposição importante para cadeias de Markov, conforme o Teorema 2.2 de Karlin e Taylor(1981). 
Proposição 1.2 Se i e j estão na mesma classe recorrente, então

$$
\lim _{n \rightarrow \infty} \frac{\sum_{m=0}^{n} P_{i j}^{m}}{\sum_{m=0}^{n} P_{i i}^{m}}=i P_{i j}{ }^{*},
$$

onde

$$
i P_{i j}{ }^{*}=\sum_{m \geq 0} i P_{i j}^{m}=\sum_{m \geq 1} P\left(X_{m}=j, X_{m-1} \neq i, \ldots, X_{1} \neq i \mid X_{0}=i\right)
$$

Note que $i P_{i j}{ }^{*}$ é o número esperado de visitas ao estado $j$ entre visitas sucessivas ao estado $i$.

Agora, fazemos algumas considerações sobre cadeias de Markov quando o espaço de estados é geral.

Definição 1.1 Seja E um conjunto qualquer. O espaço de estados E é dito geral se está equipado com uma $\sigma$-álgebra contavelmente gerada $B(E)$, isto é, gerada por uma quantidade enumerável de subconjuntos de E.

Definimos, assim, o conceito que generaliza a idéia de matriz de transição apresentada para o caso enumerável, e que, analogamente, exerce a função de lei de probabilidade de transição de um passo da cadeia.

Definição 1.2 Se $P=\{P(x, A), x \in E, A \in \mathbb{E}\}$ é tal que

a) para cada $A \in \mathbb{E}, P(., A)$ é uma função mensurável não-negativa em $E$; $e$

b) para cada $x \in E, P(x,$.$) é uma medida de probabilidade em \mathbb{E}$,

então nós chamamos $P$ um núcleo de probabilidade de transição ou função de transição de Markov.

Assim, considere uma cadeia de Markov $\left\{X_{n}\right\}_{n \geq 0}$, que tem valores em $E$, com o núcleo de transição definido acima. Ou seja, $\left\{X_{n}\right\}_{n \geq 0}$ é um processo estocástico com valores em $E$ que satisfaz:

$$
\begin{gathered}
P\left(X_{n+1} \in A \mid X_{0}, \ldots, X_{n}\right)=P\left(X_{n+1} \in A \mid X_{n}\right), \\
\mathrm{e} \\
P\left(X_{n} \in A \mid X_{0}=x\right)=P^{n}(x, A)=\int_{E} P^{m}(x, d y) P^{n-m}(y, A), \quad 1 \leq m \leq n .
\end{gathered}
$$


A equação (1.7), denominada equação de Chapman-Kolmogorov, decorre da equação (1.6), conhecida como propriedade de Markov.

Assim como no caso enumerável, é um fato conhecido que uma cadeia de Markov $\left\{X_{n}\right\}_{n>0}$ com espaço de estados geral $E$ também é completamente determinada pelo núcleo $P$ e por uma distribuição inicial $\mu_{0}(A)=P\left(X_{0} \in A\right)$. Por isso, temos que

$$
P\left(X_{0} \in A_{0}, X_{1} \in A_{1}, \ldots, X_{n} \in A_{n}\right)=\int_{A_{0}} \mu_{0}\left(d x_{0}\right) \int_{A_{1}} P\left(x_{0}, d x_{1}\right) \ldots \int_{A_{n}} P\left(x_{n-1}, d x_{n}\right) .
$$

Uma situação interessante ocorre quando $\mu_{0}(A)=\delta_{x}(A)$, onde $x \in E \mathrm{e}$

$$
\delta_{x}(A)= \begin{cases}1, & \text { se } x \in A \\ 0, & \text { se } x \notin A\end{cases}
$$

Neste caso, dizemos que o processo se inicia em $x$ e denotamos isso por

$$
P\left(X_{n} \in A \mid X_{0}=x\right)=P_{x}\left(X_{n} \in A\right) .
$$

Para finalizarmos esta seção, definimos os conceitos gerais de distribuição estacionária e distribuição de equilíbrio de uma cadeia de Markov com espaço de estados geral.

Definição 1.3 Uma medida de probabilidade $\pi$ é denominada uma distribuição estacionária para uma cadeia de Markov com núcleo de transição de probabilidade $P$, se $\pi=\pi P$.

Definição 1.4 Uma medida de probabilidade $\pi$ é denominada uma distribuição de equilíbrio se

$$
\lim _{n \rightarrow \infty}\left|P^{n}(x, A)-\pi(A)\right|=0, \quad \forall x \in E \quad \text { e } \forall A \in \mathbb{E} .
$$

\subsection{Distribuições Estáveis}

As distribuições estáveis são fundamentais para o estudo do comportamento assintótico de somas parciais de variáveis aleatórias. Nesta seção, listamos alguns fatos básicos relacionados a essa importante classe de distribuições.

Definição 1.5 Para $0<\alpha \leq 2$, dizemos que $G$ é uma distribuição $\alpha$-estável se, para qualquer $n \geq 2$, existem números reais $d_{n}$ tais que

$$
Z_{1}+Z_{2}+\ldots+Z_{n} \stackrel{d}{=} n^{\frac{1}{\alpha}} Z+d_{n}, Z \stackrel{d}{=} G,
$$

onde $Z_{1}, Z_{2}, \ldots, Z_{n}$ são cópias independentes de $Z$. 
Definição 1.6 Uma variável aleatória $G$ possui distribuição estável se existem parâmetros $\alpha \in(0,2]$, $\sigma \geq 0, \beta \in[-1,1]$ e $\mu \in \mathbb{R}$, tais que a sua função característica tem a seguinte forma:

$$
\Phi(t)=E(\exp (i t G))=\left\{\begin{array}{l}
\exp \left(-\sigma^{\alpha}|t|^{\alpha}\left(1-i \beta(\operatorname{sinal}(t)) \tan \frac{\pi \alpha}{2}\right)+i \mu t\right), \text { se } \alpha \neq 1, \\
\exp \left(-\sigma|t|\left(1+i \beta \frac{2}{\pi}(\operatorname{sinal}(t)) \ln |t|\right)+i \mu t\right), \text { se } \alpha=1 .
\end{array}\right.
$$

Se a definição acima é satisfeita, denotamos $G=S_{\alpha}(\sigma, \beta, \mu)$, onde $\sigma$ é chamado parâmetro de escala, $\beta$ é o parâmetro de assimetria e $\mu$ é o parâmetro de locação. Exemplos clássicos são a distribuição Gaussiana $N\left(\mu, 2 \sigma^{2}\right)=S_{2}(\sigma, 0, \mu)$, a distribuição Cauchy padrão $S_{1}(1,0,0)$ e a distribuição de Lèvy $S_{\frac{1}{2}}(\sigma, 0,0)$. Além disso, as distribuições estáveis constituem a única distribuição limite possível para somas parciais adequadamente estabilizadas de variáveis aleatórias independentes e identicamente distribuídas.

A proposição abaixo nos dá alguns resultados que serão necessários mais adiante (veja, por exemplo, Samorodnitsky e Taqqu (2000)).

Proposição 1.3 Assuma que $Z$ tem uma distribuição $\alpha$-estável $S_{\alpha}(\sigma, \beta, \mu)$.

a) Se $0<\alpha^{\prime}<\alpha<2$, então $E\left\{|Z|^{\alpha^{\prime}}\right\}<\infty$ e $E\left\{|Z|^{\alpha}\right\}=\infty$.

b) Se $\alpha>1$, então $E(Z)=\mu$ e as constantes $d_{n}$ em (1.8) podem ser tomadas como $d_{n}=\mu\left(n-n^{\frac{1}{\alpha}}\right)$.

c) $S e 1<\alpha \leq 2, \beta=0, a \in \mathbb{R} e b \in \mathbb{R}$, então $a Z+b \stackrel{d}{=} S_{\alpha}(|a| \sigma, 0, a \mu+b)$.

\subsection{Distância Mallows}

Conforme Mallows(1972), para $r>0$, a r-ésima distância Mallows entre duas funções de distribuição de probabilidade $F$ e $G$ é dada por

$$
d_{r}(F, G)=\inf _{(X, Y)}\left(E\left\{|X-Y|^{r}\right\}\right)^{\frac{1}{r}}, X \stackrel{d}{=} F, Y \stackrel{d}{=} G,
$$

onde o ínfimo é tomado sobre todos os vetores aleatórios $(X, Y)$ com distribuições marginais $F$ e $G$, respectivamente. Pela mesma notação, nós também escreveremos

$$
d_{r}(F, G)=d_{r}(X, Y), \quad X \stackrel{d}{=} F, Y \stackrel{d}{=} G .
$$

No espaço das funções de distribuição com r-ésimo momento finito, a distância Mallows, também conhecida como métrica de Wasserstein, satisfaz as seguintes relações métricas:

$$
d_{r}^{r}(F, G) \leq d_{r}^{r}\left(F, F_{0}\right)+d_{r}^{r}\left(F_{0}, G\right), \quad 0<r \leq 1
$$




$$
d_{r}(F, G) \leq d_{r}\left(F, F_{0}\right)+d_{r}\left(F_{0}, G\right), r \geq 1,
$$

onde $F_{0}$ é uma função de distribuição. A conexão entre convergência em distância Mallows e a convergência em distribuição foi demonstrada por Bickel e Freedman (1981).

Teorema 1.1 (Bickel e Freedman (1981)) Para $r \geq 1$ e funções de distribuição $F_{0} e\left\{F_{n}\right\}_{n \geq 1}$ satisfazendo $\int|x|^{r} d F_{j}(x)<\infty, j \geq 0$, nós temos

$$
d_{r}\left(F_{n}, F_{0}\right) \stackrel{n \rightarrow \infty}{\longrightarrow} 0 \Longleftrightarrow F_{n} \stackrel{d}{\rightarrow} F e \int|x|^{r} d F_{n}(x) \stackrel{n \rightarrow \infty}{\longrightarrow} \int|x|^{r} d F_{0}(x) .
$$

Conforme Dorea e Ferreira (2012), o seguinte teorema nos fornecerá uma maneira alternativa e simples para calcular a distância Mallows quando $r \geq 1$.

Teorema 1.2 (Dorea e Ferreira (2012)) Para $r \geq 1$, temos

$$
\begin{aligned}
d_{r}^{r}(F, G) & =E\left\{\left|F^{-}(U)-G^{-}(U)\right|^{r}\right\} \\
& =\int_{0}^{1}\left|F^{-}(U)-G^{-}(U)\right|^{r} d u \\
& =E\left\{\left|X^{*}-Y^{*}\right|^{r}\right\}=\int|x-y|^{r} d H^{*}(x, y),
\end{aligned}
$$

onde $U$ é uniformemente distribuída no intervalo $(0,1), F^{-} e G^{-}$são as funções inversas generalizadas de $F$ e $G$,

$$
F^{-}(u)=\inf \{x ; F(x) \geq u\}, 0<u<1,
$$

$e\left(X^{*}, Y^{*}\right)$ é um vetor aleatório com distribuições marginais $X^{*} \stackrel{d}{=} F, Y^{*} \stackrel{d}{=} G$ e distribuição conjunta $H^{*}$ dada por

$$
H^{*}(x, y)=P\left(X^{*} \leq x, Y^{*} \leq y\right)=F(x) \wedge G(y)
$$

$\operatorname{com} u \wedge v=\min (u, v)$.

A distância Mallows tem sido sucessivamente usada para obter teoremas do tipo limite central para distribuições $\alpha$-estáveis. Assim, citamos os resultados de Johnson e Samworth (2005) para o caso Gaussiano, e os resultados do tipo Lindeberg em Barbosa e Dorea (2009) para o caso não-Gaussiano. 
Teorema 1.3 (Johnson e Samworth (2005)) Sejam $\xi, \xi_{1}, \xi_{2} \ldots$, variáveis aleatórias independentes e identicamente distribuídas. Assuma que $0<\operatorname{var}(\xi)<\infty$ e que para algum $r \geq 2$ temos $d_{r}(\xi, Z)<\infty$ $\operatorname{com} Z \stackrel{d}{=} S_{2}(\sigma, 0, \mu)$. Então

$$
d_{r}\left(\frac{\xi_{1}+\ldots+\xi_{n}}{\sqrt{\operatorname{nvar}(\xi)}}, Z_{0}\right) \stackrel{n \rightarrow \infty}{\longrightarrow} 0
$$

onde $Z_{0} \stackrel{d}{=} S_{2}(1,0,0)=N(0,1)$.

Teorema 1.4 (Barbosa e Dorea (2009)) Sejam $0<\alpha<2 e\left\{\left(\xi_{n}, Z_{n}\right)\right\}_{n \geq 1}$ uma sequência de vetores aleatórios independentes tais que $Z_{1}, Z_{2}, \ldots$, são cópias independentes de uma variável aleatória $\alpha$ estável $Z \stackrel{d}{=} S_{\alpha}(\sigma, 0, \mu)$. Assuma que para todo $b>0$ nós temos

$$
\lim _{n \rightarrow \infty} \frac{1}{n} \sum_{k=1}^{n} E\left\{\left|\xi_{k}-Z_{k}\right|^{\alpha} I_{\left(\left|\xi_{k}-Z_{k}\right|>b n^{\frac{2-\alpha}{2 \alpha}}\right)}\right\}=0
$$

Então, existe uma sequência de constantes $\left\{c_{n}\right\}_{n \geq 1}$ tal que

$$
d_{\alpha}\left(\frac{\xi_{1}+\ldots+\xi_{n}-c_{n}}{n^{\frac{1}{\alpha}}}, Z\right) \stackrel{n \rightarrow \infty}{\longrightarrow} 0 .
$$

Além disso, se $1<\alpha<2$ então podemos tomar $c_{n}+\mu=\sum_{k=1}^{n} E\left(\xi_{k}\right)$.

\subsection{Resultados Relacionados}

O Teorema 1.1 e o Lema 1.1 abaixo indicam a conexão entre distância Mallows e integrabilidade uniforme.

Definição 1.7 Uma sequência de variáveis aleatórias $\left\{\xi_{n}\right\}_{n \geq 1}$ é dita uniformemente integrável se

$$
\lim _{c \rightarrow \infty} \sup _{n} \int_{\left(\left|\xi_{n}\right|>c\right)}\left|\xi_{n}\right| d P=0
$$

Pelo Teorema 5.4 em Billingsley (1968), nós temos

Lema 1.1 (Billingsley (1968)) Suponha que $\xi_{n} \stackrel{d}{\rightarrow} \xi_{0}$. Se $\{\xi\}_{n \geq 1}$ são uniformemente integráveis, então $E\left(\xi_{n}\right) \stackrel{n \rightarrow \infty}{\longrightarrow} E\left(\xi_{0}\right)$. Condições suficientes para integrabilidade uniforme são dadas por

a) Para algum $\epsilon>0$, nós temos $\sup _{n \geq 1} E\left\{\left|\xi_{n}\right|^{1+\epsilon}\right\}<\infty$;

ou 
b) $\xi_{0}$ e $\xi_{n}$ são não-negativos e $E\left(\xi_{n}\right) \stackrel{n \rightarrow \infty}{\longrightarrow} E\left(\xi_{0}\right)$.

Definição 1.8 Para uma sequência de variáveis aleatórias $\left\{X_{n}\right\}_{n \geq 1} e \Im_{n}=\sigma\left(X_{i} ; i=1, \ldots, n\right)$, dizemos que $\left\{X_{n}, \Im_{n}\right\}_{n \geq 1}$ é uma martingale se, para cada $n$, estão satisfeitas as condições abaixo:

a) $\Im_{n} \subset \Im_{n+1}$ e $X_{n}$ é $\Im_{n}$-mensurável;

b) $E\left|X_{n}\right|<\infty$;

c) $X_{n}=E\left(X_{n+1} \mid \Im_{n}\right)$, q.c. .

Martingales também estão relacionadas à integrabilidade uniforme. O Lema abaixo é o Teorema 2.22 de Hall e Heyde (1980).

Lema 1.2 (Hall e Heyde (1980)) Sejam $\left\{\sum_{k=1}^{n} \xi_{k}, \Im_{n}\right\}_{n \geq 1}$ uma martingale e $1 \leq r<2$. Se $\left\{\left|\xi_{n}\right|^{r}\right\}_{n \geq 1}$ é uniformemente integrável, então

$$
\lim _{n \rightarrow \infty} \frac{1}{n} E\left\{\left|\sum_{k=1}^{n} \xi_{k}\right|^{r}\right\}=0
$$

A seguir, listamos algumas desigualdades de momentos que serão necessárias para nossas demonstrações, dentre elas a desigualdade de Burkholder em (1.17) e algumas desigualdades de Martingales (veja Hall e Heyde, 1980).

Lema 1.3 a) Para variáveis aleatórias $\xi_{1}, \xi_{2}, \ldots$, nós temos

$$
E\left\{\left|\sum_{k=1}^{n} \xi_{k}\right|^{r}\right\} \leq \sum_{k=1}^{n} E\left\{\left|\xi_{k}\right|^{r}\right\}, \text { para } 0<r \leq 1
$$

$e$

$$
E\left\{\left|\sum_{k=1}^{n} \xi_{k}\right|^{r}\right\} \leq n^{r-1} \sum_{k=1}^{n} E\left\{\left|\xi_{k}\right|^{r}\right\}, \text { para } r \geq 1 .
$$

Se, em adição, $\xi_{1}, \xi_{2}, \ldots$, são independentes, então

$$
E\left\{\left|\sum_{k=1}^{n}\left(\xi_{k}-E\left(\xi_{k}\right)\right)\right|^{r}\right\} \leq 2 \sum_{k=1}^{n} E\left\{\left|\xi_{k}-E\left(\xi_{k}\right)\right|^{r}\right\}, \text { para } 1 \leq r \leq 2 .
$$


b) (Burkholder (1973)) Se $\left\{\sum_{k=1}^{n} \xi_{k}, \Im_{n}\right\}_{n \geq 1}$ é uma martingale, existe uma constante $C(r)>0$ tal que

$$
E\left\{\left|\sum_{k=1}^{n} \xi_{k}\right|^{r}\right\} \leq C(r) E\left\{\left(\sum_{k=1}^{n} \xi_{k}^{2}\right)^{\frac{r}{2}}\right\} \text {, para } r>1 .
$$

Para $\lambda>0$, nós temos a clássica desigualdade de Kolmogorov:

$$
P\left\{\max _{1 \leq m \leq n}\left|\sum_{k=1}^{m} \xi_{k}\right|>\lambda\right\} \leq \frac{1}{\lambda^{r}} E\left\{\left|\sum_{k=1}^{n} \xi_{k}\right|^{r}\right\}, \quad \text { para } r \geq 1
$$

Além disso, para $p>1 e \frac{1}{p}+\frac{1}{q}=1$, nós temos

$$
\left(E\left\{\left|\sum_{k=1}^{n} \xi_{k}\right|^{p}\right\}\right)^{\frac{1}{p}} \leq\left(E\left\{\max _{1 \leq m \leq n}\left|\sum_{k=1}^{m} \xi_{k}\right|^{p}\right\}\right)^{\frac{1}{p}} \leq q\left(E\left\{\left|\sum_{k=1}^{n} \xi_{k}\right|^{p}\right\}\right)^{\frac{1}{p}}
$$

Nós obteremos resultados relacionados à convergência em distribuição de somas parcias aleatoriamente indexadas e para isso a condição de Ascombe para preservação da convergência fraca será usada (para detalhes, veja, por exemplo, Dorea, David e Werner (1984)).

Lema 1.4 (Dorea, David e Werner (1984)) Suponha que $\xi_{n} \stackrel{d}{\rightarrow} \xi_{0}$ e seja $\left\{\nu_{n}\right\}_{n \geq 0}$ uma sequência de índices aleatórios para a qual $\frac{\nu_{n}}{n} \stackrel{p}{\rightarrow} \gamma>0$. Se dado $\epsilon>0$ e $\eta>0$, existem $N_{0}(\epsilon, \eta)$ e $\delta_{0}(\epsilon, \eta)>0$ tais que para $n \geq N_{0}(\epsilon, \eta)$ nós temos

$$
P\left(\max _{|k-n| \leq \delta n}\left|\xi_{k}-\xi_{n}\right| \geq \epsilon\right) \leq \eta
$$

então $\xi_{\nu_{n}} \stackrel{d}{\rightarrow} \xi_{0}$

\subsection{Processos Empíricos}

Enunciamos, nesta seção, definições e resultados básicos acerca de processos empíricos. Maiores detalhes podem ser encontrados em Csörgo e Révész (1981) ou em Shorack e Wellner (1986). 
Definição 1.9 Sejam $X_{1}, \ldots, X_{n}$ variáveis aleatórias. Para cada natural $n \geq 1$, definimos a $n$-ésima função de distribuição empírica associada a $X_{1}, X_{2}, \ldots, X_{n}$ como

$$
F_{n}(x, \omega)=\frac{\sum_{i=1}^{n} \mathbb{I}_{\left(X_{i}(\omega) \leq x\right)}}{n} .
$$

Se fixarmos $x$, a função acima é uma variável aleatória e o numerador representa o número de índices $i^{\prime}$ s que satisfazem $X_{i}(\omega) \leq x$. Por isso, $F_{n}(x)$ também pode ser vista como a proporção de valores da amostra que são menores ou iguais a $x$.

Definição 1.10 Definimos o Processo Empírico, referente às variáveis aleatórias $X_{1}, \ldots, X_{n}$ com uma função de distribuição comum $F$, como

$$
\beta_{n}(x)=\sqrt{n}\left(F_{n}(x)-F(x)\right), \quad x \in \mathbb{R}, n \geq 1 .
$$

Além disso, é também fundamental para a obtenção de nossos resultados a versão uniforme do processo empírico, que definimos a seguir.

Definição 1.11 O processo empírico uniforme é dado por

$$
u_{n}(t)=\sqrt{n}\left(U_{n}(t)-t\right), \quad t \in[0,1], n \geq 1,
$$

onde $U_{n}(t)$ é a distribuição empírica uniforme.

Cabe observarmos que, se tomarmos $F$ contínua, então

$$
\beta_{n}(x)=u_{n}(F(x)) .
$$

Essas definições instigam uma reflexão acerca de uma possível associação entre $\beta_{n}(x)$ e a distribuição Gaussiana, tendo em vista o Teorema do Limite Central. De fato, o processo empírico tem relação direta com uma variável aleatória Gaussiana específica, que é chamada Ponte Browniana.

Definição 1.12 Um processo estocástico $\{B(t), 0 \leq t \leq 1\}$ é uma Ponte Browniana se

$$
B(t)=\tilde{W}(t)-t \tilde{W}(1), 0 \leq t \leq 1,
$$

em que $\{\tilde{W}(t), 0 \leq t<\infty\}$ é um Movimento Browniano.

É bem conhecido que $B(t)$ é um processo Gaussiano e $E(B(t))=0$. Além disso, para $s, t \in[0,1)$,

$$
\begin{aligned}
\operatorname{Cov}[B(s), B(t)]= & \operatorname{Cov}[\tilde{W}(s)-s \tilde{W}(1), \tilde{W}(t)-t \tilde{W}(1)] \\
= & E\{[\tilde{W}(s)-s \tilde{W}(1)] \cdot[\tilde{W}(t)-t \tilde{W}(1)]\} \\
& -E[\tilde{W}(s)-s \tilde{W}(1)] \cdot E[\tilde{W}(t)-t \tilde{W}(1)] \\
= & E\left[\tilde{W}(s) \tilde{W}(t)-t \tilde{W}(s) \tilde{W}(1)-s \tilde{W}(t) \tilde{W}(1)+s t(\tilde{W}(1))^{2}\right] \\
= & \min \{s, t\}-s t=(s \wedge t)-s t .
\end{aligned}
$$


Portanto, $\operatorname{Var}(B(t))=t(1-t)$, o que evidencia a Ponte Browniana como um processo Gaussiano com média zero e função covariância " $(s \wedge t)-s t$ ".

A propósito, o Teorema do Limite Central para processos empíricos é enunciado da seguinte maneira:

Teorema 1.5 Sejam $X_{1}, \ldots, X_{n}$ variáveis aleatórias i.i.d. com função de distribuição $F$. Se $x \in \mathbb{R} e ́$ tal que $0<F(x)<1$, então

$$
\beta_{n}(x) \stackrel{d}{\longrightarrow} Z(x) \stackrel{d}{=} N(0, F(x)(1-F(x))) .
$$

No Capítulo 3 provaremos que para cadeias de Markov gerais e ergódicas valerá a convergência de $\beta_{n}(x)$ para a Ponte Browniana $B(F(x))$ no sentido de processos.

Na sequência, abordamos resumidamente conceitos básicos sobre o processo quantil empírico, um processo estocástico que pode ser considerado como o inverso do processo empírico $\beta_{n}(x)$.

Definição 1.13 Sejam $X_{1}, \ldots, X_{n}$ variáveis aleatórias com uma função de distribuição $F$, então

a) A inversa generalizada de F é a função dada por

$$
F^{-}(t)=\inf \{x ; F(x) \geq t\}, \quad F^{-}(0)=F^{-}\left(0^{+}\right) ;
$$

b) A inversa generalizada empírica ou função quantil empírica é dada por

$$
F_{n}^{-}(t, \omega)=\inf \left\{x ; F_{n}(x, \omega) \geq t\right\} .
$$

Da mesma maneira como para $F_{n}$, associamos à inversa empírica $F_{n}^{-}$um processo estocástico.

Definição 1.14 Definimos o processo quantil empírico, associado às variáveis aleatórias $X_{1}, \ldots, X_{n}$ com uma função de distribuição comum $F$, como

$$
q_{n}(t)=\sqrt{n}\left(F_{n}^{-}(t)-F^{-}(t)\right), t \in(0,1), n \geq 1 .
$$

Analogamente ao caso empírico, há também a versão uniforme do processo quantil empírico, que definimos a seguir.

Definição 1.15 O processo quantil empírico uniforme é dado por

$$
v_{n}(t)=\sqrt{n}\left(U_{n}^{-}(t)-t\right), \quad t \in[0,1], n \geq 1,
$$

onde $U_{n}^{-}(t)$ é a distribuição quantil empírica uniforme.

Além disso, temos que

$$
q_{n}(t)=\sqrt{n}\left(F^{-}\left(U_{n}^{-}(t)\right)-F^{-}(t)\right)
$$




$$
q_{n}(t)=\sqrt{n}\left(U_{n}^{-}(t)-t\right) \cdot\left[F^{-}\left(\xi_{n}\right)\right]^{\prime},
$$

onde $t \wedge U_{n}{ }^{-}(t) \leq \xi_{n} \leq t \vee U_{n}{ }^{-}(t)$ e $\left[F^{-}\left(\xi_{n}\right)\right]^{\prime}$ é a derivada de $F^{-}($.$) aplicada em \xi_{n}$. Observe que podemos considerar $\xi_{n}=t$.

Embora $F_{n}$ funcione como bom estimador para a distribuição $F$, a distribuição quantil empírica não funciona como um bom estimador para a inversa generalizada $F^{-}$, exceto nos pontos de continuidade desta, o que já restringe qualquer analogia que possa ser feita com $\beta_{n}(x)$. No caso i.i.d., o próximo teorema confirma esse raciocínio ao mostrar que, assintoticamente, o comportamento de $q_{n}(t)$ está bem caracterizado, mas com hipóteses mais restritivas do que as que temos para o comportamento assintótico de $\beta_{n}(x)$.

Teorema 1.6 (Shorack e Wellner (1986), p. 639) . Sejam $X_{1}, \ldots, X_{n}$ variáveis aleatórias i.i.d. tais que $F$ é uma função de distribuição derivável em $F^{-}(t), t \in(0,1)$, e satisfaz a condição $F^{\prime}\left(F^{-}(t)\right)=$ $\frac{1}{f\left(F^{-}(t)\right)}>0$, com $f$ sendo a densidade de probabilidade associada a $F$. Neste caso, é verdade que

$$
q_{n}(t) \stackrel{d}{\longrightarrow} \frac{B(t)}{f\left(F^{-}(t)\right)} \stackrel{d}{=} \mathcal{N}\left(0, \frac{t(1-t)}{f^{2}\left(F^{-}(t)\right)}\right)
$$

\subsection{Sobre Convergência Fraca}

Nesta seção, inicialmente apresentamos um resumo de resultados básicos relativos à convergência fraca de sequências de medidas de probabilidade, estabelecendo, em seguida, condições suficientes para a convergência fraca em um espaço métrico particular, o espaço $D[0,1]$ com a topologia de Skorohod. Estes assuntos são tratados com detalhes nos capítulos 1 e 3 de Billingsley (1968). Aqui nós nos limitaremos a enunciar alguns resultados e definições contidos nessa referência bibliográfica, com o objetivo de facilitar a compreensão do conteúdo. Em seguida, abordamos importantes resultados que nos permitem usar a convergência fraca do processo empírico de maneira a obter a convergência fraca para o caso quantil.

No que se segue, $S$ e $S^{\prime}$ representam espaços métricos quaisquer e $\mathbf{S}$ a $\sigma$-álgebra de Borel que lhes está associada.

Definição 1.16 Sejam $P_{n}, n \in \mathbb{N}$, e $P$ medidas de probabilidade em $(S, \mathbf{S})$. Dizemos que $\left\{P_{n}\right\}_{n \geq 1}$ converge fracamente para $P$ e escrevemos $P_{n} \Rightarrow P$, se $\int f d P_{n}$ converge para $\int f d P$, para toda função $f$ real contínua e limitada definida em $S$.

Consideramos agora uma sequência $\left\{X_{n}\right\}_{n \geq 1}$ de elementos aleatórios em $(S, \mathbf{S})$ e $\left\{P_{n}\right\}_{n \geq 1}$ a sequência das respectivas distribuições.

Definição 1.17 Dizemos que $\left\{X_{n}\right\}_{n \geq 1}$ converge em distribuição para o elemento aleatório $X$, e escrevemos $X_{n} \stackrel{D}{\longrightarrow} X$, se $\left\{P_{n}\right\}_{n \geq 1}$ converge fracamente para a distribuição de $X$. 
Tendo em conta a definição anterior, todos os resultados a seguir relativos à convergência fraca podem ser reescritos em termos de convergência em distribuição de variáveis aleatórias. Além disso, $X_{n} \Rightarrow X$ significa que a sequência de medidas de probabilidade relativas a $X_{n}$ converge fracamente para a medida de probabilidade associada a $X$.

Teorema 1.7 (Billingsley (1968)) Seja g uma função mensurável de $S$ em $S^{\prime}$ e $X_{n} \Rightarrow X$. Se o conjunto de descontinuidades de g tiver medida P nula, então $g\left(X_{n}\right) \Rightarrow g(X)$.

A seguir, introduzimos dois conceitos básicos para o estudo da convergência fraca em espaços métricos.

Definição 1.18 Um conjunto $\prod$ de medidas de probabilidade em $(S, \mathbf{S})$ é rígido se, para todo $\epsilon>0$, existe um conjunto compacto $K$ tal que $P(K)>1-\epsilon$, para toda $P$ de $\prod$.

Particularizamos, agora, o nosso estudo, considerando o espaço métrico $D[0,1]$, que é o espaço das funções reais definidas em $[0,1]$ que são contínuas pela direita e têm limite finito à esquerda.

O teorema a seguir estabelece condições suficientes para a convergência fraca em $D[0,1]$.

Teorema 1.8 (Billingsley (1968)) Se $\left\{P_{n}\right\}_{n \geq 1}$ é uma sequência rígida de medidas de probabilidade em $D[0,1]$ e as distribuições finitas $\left\{P_{n}\right\}_{n \geq 1}$ convergem fracamente para as distribuições finitas de $P$ , então $P_{n} \Rightarrow P$.

Por este teorema, para demonstrar a convergência fraca de uma sequência $\left\{P_{n}\right\}_{n \geq 1}$, é suficiente mostrar que $\left\{P_{n}\right\}_{n \geq 1}$ é rígida e que as suas distribuições finitas convergem fracamente. Além disso, nessa abordagem podemos sempre considerar a sequência $\left\{X_{n}\right\}_{n \geq 1}$ associada a $\left\{P_{n}\right\}_{n \geq 1}$. O seguinte teorema estabelece condições suficientes para a rigidez de uma sequência $\left\{X_{n}(.)\right\}_{n \geq 1}$. Trata-se de uma versão do Teorema 15.5 de Billingsley (1968).

Teorema 1.9 (Billingsley (1968)) Uma sequência $\left\{X_{n}(.)\right\}_{n \geq 1}$ é rígida se as seguintes condições são satisfeitas:

a) para cada $\eta>0$, existe um $a \in \mathbb{R}$ tal que

$$
P\left\{\left|X_{n}(0)\right|>a\right\} \leq \eta, \quad n \geq 1
$$

b) Para cada $\eta$ e $\epsilon$ positivos, existem um $\delta$, com $0<\delta<1$, e um inteiro $n_{0}$ tais que

$$
P\left\{\sup _{t \leq s \leq t+\delta}\left|X_{n}(s)-X_{n}(t)\right| \geq \epsilon\right\} \leq \delta \eta, \quad n \geq n_{0},
$$

para todo t. 
O Teorema 1.8 abaixo mostra que a convergência fraca das distribuições finitas de $X_{n}$ é equivalente à convergência em distribuição da sequência de vetores aleatórios $\left(X_{n}\left(t_{1}\right), \ldots, X_{n}\left(t_{k}\right)\right)$. Assim, o resultado a seguir é uma das principais ferramentas na tentativa de provar a convergência das distribuições de dimensão finita de um processo estocástico.

Teorema 1.10 (Cràmer-Wold) Sejam $X_{n}=\left(X_{n}{ }^{(1)}, X_{n}{ }^{(2)}, \ldots, X_{n}{ }^{(k)}\right)$ e $X=\left(X^{(1)}, X^{(2)}, \ldots, X^{(k)}\right)$ vetores aleatórios $k$-dimensionais. Então

$$
X_{n} \stackrel{D}{\longrightarrow} X
$$

se, e somente se, para todo $\left(t_{1}, t_{2}, \ldots, t_{k}\right) \in \mathbb{R}^{k}$, ocorre

$$
\sum_{j=1}^{k} t_{j} X_{n}{ }^{(j)} \stackrel{D}{\longrightarrow} \sum_{j=1}^{k} t_{j} X^{(j)} .
$$

Para obtermos a convergência fraca do processo quantil empírico, objeto de estudo da segunda seção do capítulo 3, enunciamos a seguir alguns importantes resultados e definições.

Definição 1.19 Se $\left\{f_{n}, n \geq 0\right\}$ são funções reais definidas em $\mathbb{R}$, então $f_{n}$ converge uniformemente para $f_{0}$ em $A \subset \mathbb{R}$ se

$$
\sup _{x \in A}\left|f_{n}(x)-f_{0}(x)\right| \longrightarrow 0, n \longrightarrow \infty .
$$

Para funções em $\mathbb{R}$, convergir localmente uniformemente (l.u.) significa que (1.27) vale para qualquer intervalo compacto A.

Observação 1.1 Conforme Billingsley (1968), se uma função $x_{n}(t) \stackrel{n \longrightarrow \infty}{\longrightarrow} x(t)$ na topologia Skorohod, e a função limite $x(t)$ é contínua (definida em um compacto), então, equivalentemente, $x_{n}(t) \stackrel{n \longrightarrow \infty}{\longrightarrow}$ $x(t)$ (localmente) uniformemente.

Teorema 1.11 (Skorohod) Sejam $X, X_{1}, X_{2}, \ldots$ elementos aleatórios em um espaço métrico separável $S$ tais que $X_{n} \stackrel{D}{\longrightarrow} X$. Então existem elementos aleatórios $X^{\prime}, X_{1}{ }^{\prime}, X_{2}{ }^{\prime}, \ldots$ definidos em um mesmo espaço de probabilidade e tendo as mesmas distribuições de probabilidade que $X, X_{1}, X_{2}, \ldots$, tais que $X_{n}^{\prime} \stackrel{\text { q.c. }}{\longrightarrow} X^{\prime}$.

Além disso, a seguir apresentamos um lema que associado ao Teorema 1.11, junto à relação explicitada na Observação 1.1, permite-nos obter a convergência fraca do processo quantil empírico, dado que já obtivemos o resultado para o caso empírico. Esse lema é uma adaptação do Lema 1 de Vervaat (1972). Para mais detalhes e sua demonstração, veja Resnick (2007) e Vervaat (1971). 
Lema 1.5 (Vervaat, 1971) Suponha que para cada $n, x_{n} \in D[0,1]$ é uma função não-decrescente e $x_{0} \in C[0,1]$. Se $c_{n} \rightarrow \infty e$

$$
c_{n}\left(x_{n}(t)-t\right) \stackrel{n \rightarrow \infty}{\longrightarrow} x_{0}(t),
$$

localmente uniformemente, então também

$$
c_{n}\left(x_{n}{ }^{-1}(t)-t\right) \stackrel{n \rightarrow \infty}{\longrightarrow}-x_{0}(t),
$$

localmente uniformemente. 


\section{Capítulo 2}

\section{Comportamento Assintótico de Cadeias de Markov via Distância Mallows}

\subsection{Introdução}

Neste capítulo analisamos o comportamento assintótico de cadeias de Markov $X=\left\{X_{n}\right\}_{n \geq 0}$ ergódicas, com um espaço de estados enumerável $S$. Nós consideraremos funcionais da cadeia no sentido que para $\phi: S \rightarrow \mathbb{R}$ obteremos a convergência das somas parciais $S_{n}=\sum_{l=0}^{n} \phi\left(X_{l}\right)$. A distribuição assintótica de somas parciais de variáveis aleatórias independentes e identicamente distribuídas está intimamente relacionada às distribuições $\alpha$-estáveis, já que essas constituem a única distribuição limite possível para somas parciais estabilizadas $\frac{S_{n}-b_{n}}{a_{n}}$. De fato, quando $\alpha<2$, a relação entre distribuições $\alpha$-estáveis com somas parciais de variáveis aleatórias i.i.d. de cauda pesada é análoga ao que ocorre com a distribuição normal e as variáveis aleatórias com segundo momento finito.

Nosso estudo será subdividido em dois casos: o caso Gaussiano $(\alpha=2)$ e o caso Cauda-Pesada com média finita $(1<\alpha<2)$.

Na seção 2.3 abordamos o caso Gaussiano. Nossos Teoremas 2.1 e 2.4 estabelecem condições sob as quais, além da convergência em distribuição, também obtemos a convergência em distância Mallows bem como a convergência dos momentos existentes. Esses resultados podem ser visualizados como uma extensão do Teorema do Limite Central (TLC) para cadeias de Markov (conforme Teorema 1, Cap. 16, Chung (1960) ). Nosso corolário 2.1 pode ser enxergado como uma prova alternativa para o TLC de Chung.

Na Seção 2.2, alguns resultados preliminares são obtidos. Além disso, a conexão entre nossos estudos e a distância Mallows está ilustrada no Lema 2.3. Nosso Lema 2.4 estabelece condições sob as quais somas parciais aleatoriamente indexadas preservam a convergência em distribuição.

Na Seção 2.4 consideramos o caso Cauda-Pesada, $1<\alpha<2$. Assim, nós adicionalmente exploramos a conexão entre distância Mallows e a convergência para distribuições estáveis. É impor- 
tante mencionar que a distância Mallows é de particular interesse no estudo de variáveis de CaudaPesada. Para $1<\alpha<2$ seu uso constitui uma técnica alternativa para obter resultados do tipo TLC. Nosso exemplo 2.1 ilustra uma cadeia de Markov com distribuição limite de Cauda-Pesada. Os Teoremas 2.5 e 2.8 fornecem a convergência em distância Mallows e em distribuição quando, para algum $1<\alpha<2$, uma variável aleatória $\alpha$-estável $Z$ existe tal que $d_{\alpha}\left(\sum_{l=\tau_{k}(i)}^{\tau_{k+1}(i)-1}\left(\phi\left(X_{l}\right)-\mu_{\phi}\right), Z\right)<\infty$, onde $\sum_{l=\tau_{k}(i)}^{\tau_{k+1}(i)-1}\left(\phi\left(X_{l}\right)-\mu_{\phi}\right)$ denota as somas parciais entre duas visitas consecutivas da cadeia de Markov ao estado $i$, e $\mu_{\phi}=E_{\pi}\left\{\phi\left(X_{k}\right)\right\}$, onde $0 \leq k \leq n$ e $\pi$ é a distribuição de equilíbrio. Além disso, com a condição adicional $d_{\alpha}\left(\phi\left(X_{0}\right), Z\right)<\infty$, estendemos esses resultados para o caso geral.

\subsection{Resultados Preliminares}

Nós consideraremos uma cadeia de Markov aperiódica e recorrente positiva $X=\left\{X_{n}\right\}_{n \geq 0}$ com espaço de estados enumerável $S$, probabilidade de transição $P=\left(P_{i j}\right)_{i \in S, j \in S}$ e distribuição limite $\pi=\left(\pi_{i}\right)_{i \in S}$. Seja $i \in S$ um estado arbitrário fixado e denote por $\tau_{k}(i)$ o k-ésimo tempo de visita ao estado $i$, ou seja,

$$
0=\tau_{0}(i)<\tau_{1}(i)<\tau_{2}(i)<\ldots<\tau_{k}(i)<\ldots,
$$

onde

$$
\tau_{1}(i)=\inf \left\{n ; n>0, X_{n}=i\right\}
$$

e

$$
\tau_{k+1}(i)=\inf \left\{n ; n>\tau_{k}(i), X_{n}=i\right\}, k \geq 1 .
$$

Note que nós não estamos assumindo $X_{0}=i$ e, para fins de notação, às vezes simplesmente escreveremos $\tau_{k}$ ao invés de $\tau_{k}(i)$.

Seja $\phi: S \rightarrow \mathbb{R}$ e considere a soma parcial $S_{n}=\sum_{l=0}^{n} \phi\left(X_{l}\right)$. Para estudarmos o comportamento assintótico de $S_{n}$, nós usaremos a fórmula de decomposição (dissecção) em Chung (1960), dada por

$$
S_{n}=\sum_{l=0}^{n} \phi\left(X_{l}\right)=\sum_{l=0}^{\tau_{1}-1} \phi\left(X_{l}\right)+\sum_{k=1}^{l_{n}-1}\left[\sum_{l=\tau_{k}}^{\tau_{k+1}-1} \phi\left(X_{l}\right)\right]+\sum_{l=\tau_{l n}}^{n} \phi\left(X_{l}\right),
$$

onde

$$
l_{n}=\sup \left\{k ; \tau_{k}(i) \leq n\right\} .
$$


Observe que temos $\tau_{l_{n}}(i) \leq n<\tau_{l_{n}+1}(i)$ e, como a cadeia é recorrente, também temos $\tau_{l_{n}}(i) \stackrel{n \rightarrow \infty}{\longrightarrow}$ $\infty$. Além disso, se assumirmos que $E_{\pi}\left\{\left|\phi\left(X_{k}\right)\right|\right\}=\sum_{j \in S}|\phi(j)| \pi_{j}<\infty$, podemos escrever, para $\mu_{\phi}=$ $E_{\pi}\left\{\phi\left(X_{k}\right)\right\}$

$$
S_{n}-n \mu_{\phi}=\sum_{l=0}^{\tau_{1}(i)-1}\left(\phi\left(X_{l}\right)-\mu_{\phi}\right)+\sum_{k=1}^{l_{n}-1} Y_{k}(i)+\sum_{l=\tau_{l_{n}}(i)}^{n}\left(\phi\left(X_{l}\right)-\mu_{\phi}\right),
$$

onde

$$
Y_{k}(i)=\sum_{l=\tau_{k}(i)}^{\tau_{k+1}(i)-1}\left(\phi\left(X_{l}\right)-\mu_{\phi}\right)
$$

Lema 2.1 Seja X uma cadeia de Markov aperiódica e recorrente positiva e assuma que $E_{\pi}\left\{\left|\phi\left(X_{k}\right)\right|\right\}<\infty$, $0 \leq k \leq n$. Então para todo $i \in S$,

a) $\left\{\tau_{k+1}(i)-\tau_{k}(i)\right\}_{k \geq 1}$ é uma sequência de variáveis aleatórias i.i.d. com $E\left\{\tau_{k+1}(i)-\tau_{k}(i)\right\}=$ $\frac{1}{\pi_{i}}<\infty$. E, se $n \rightarrow \infty$,

$$
\frac{\tau_{k+1}-\tau_{k}}{n} \stackrel{q . c .}{\longrightarrow} 0, \quad \frac{l_{n}}{n} \stackrel{q . c .}{\longrightarrow} \pi_{i}, \quad \frac{\tau_{l_{n}}}{l_{n}} \stackrel{\text { q.c. }}{\longrightarrow} \frac{1}{\pi_{i}} \text { e } \frac{n-\tau_{l_{n}}}{n} \stackrel{\text { q.c. }}{\longrightarrow} 0 .
$$

Além disso, $n-\tau_{l_{n}}$ é limitado em probabilidade, no sentido de que dado $\epsilon>0$ existem $N_{0}(\epsilon) e$ $K(\epsilon)$ tais que

$$
P\left(n-\tau_{l_{n}} \geq k\right) \leq \epsilon, \quad n \geq N_{0}(\epsilon), k \geq K(\epsilon) .
$$

b) Se $r \geq 1$ e $E\left\{\left(\tau_{k+1}(i)-\tau_{k}(i)\right)^{r}\right\}<\infty$, então

$$
\frac{\left(\tau_{k+1}(i)-\tau_{k}(i)\right)^{r}}{n} \stackrel{\text { q.c. }}{\longrightarrow} 0 \quad e \quad \frac{\left(\tau_{1}(i)\right)^{r}}{n} \stackrel{\text { q.c. }}{\longrightarrow} 0 .
$$

c) $\left\{Y_{k}(i)\right\}_{k \geq 1}$ é uma sequência de variáveis aleatórias i.i.d. e, para $\mu_{\phi}=E_{\pi}\left\{\phi\left(X_{0}\right)\right\}$, temos

$$
E\left\{Y_{k}(i)\right\}=0 \quad e \quad E\left\{\sum_{l=\tau_{k}(i)}^{\tau_{k+1}(i)-1} \phi\left(X_{l}\right)\right\}=\frac{1}{\pi_{i}} \mu_{\phi}
$$

Além disso, se $E_{\pi}\left\{\left|\Psi\left(X_{0}\right)\right|\right\}<\infty, \Psi: S \rightarrow \mathbb{R}$, então $E\left\{\sum_{l=\tau_{k}(i)}^{\tau_{k+1}(i)-1} \Psi\left(X_{l}\right)\right\}=\frac{1}{\pi_{i}} E_{\pi}\left\{\Psi\left(X_{0}\right)\right\}$. 
Demonstração. (a) Como a cadeia é recorrente positiva, temos $\frac{1}{\pi_{i}}<\infty$ e, pelo item (a) da Proposição 1.1, $\left\{\tau_{k+1}(i)-\tau_{k}(i)\right\}_{k \geq 1}$ é uma sequência de variáveis aleatórias i.i.d.. As convergências quase-certas (2.4) podem ser encontradas em Chung (1960). Em todo caso, é bastante simples mostrar, por exemplo, que $\frac{\tau_{n}}{n} \stackrel{q . c .}{\longrightarrow} \frac{1}{\pi_{i}}$. De fato, escreva

$$
\tau_{n}=\tau_{n}-\tau_{n-1}+\tau_{n-1}-\tau_{n-2}+\ldots+\tau_{2}-\tau_{1}+\tau_{1} .
$$

Usando o fato de que $\left\{\tau_{k+1}-\tau_{k}\right\}$ é uma sequência de variáveis aleatórias i.i.d. com média finita, uma aplicação direta da Lei Forte dos Grandes Números nos dá a convergência desejada.

Além disso, a desigualdade (2.5) é justamente o Teorema 2 do Capítulo 14 em Chung (1960).

(b) Para provarmos (2.6), notemos que

$$
E\left\{\left(\tau_{k+1}-\tau_{k}\right)^{r}\right\} \geq \sum_{n \geq 1} P\left(\left(\tau_{k+1}-\tau_{k}\right)^{r} \geq n\right) .
$$

Segue que, para $\epsilon>0$,

$$
\sum_{n \geq 1} P\left(\left(\tau_{k+1}-\tau_{k}\right)^{r} \geq \epsilon n\right) \leq \frac{E\left\{\left(\tau_{k+1}-\tau_{k}\right)^{r}\right\}}{\epsilon}<\infty .
$$

Por Borel-Cantelli, se $\left\{E_{n}\right\}$ é uma sequência de eventos tais que $\sum_{n \geq 1} P\left(E_{n}\right)<\infty$, então $P\left(\bigcap_{m \geq 1} \bigcup_{n \geq m} E_{n}\right)=$ 0. Desta forma, ao considerarmos $E_{n}=\left(\left(\tau_{k+1}-\tau_{k}\right)^{r} \geq \epsilon n\right)$, temos que

$$
P\left(\bigcap_{m \geq 1} \bigcup_{n \geq m}\left(\frac{\left(\tau_{k+1}-\tau_{k}\right)^{r}}{n} \geq \epsilon\right)\right)=0
$$

$\operatorname{Logo}, \frac{\left(\tau_{k+1}-\tau_{k}\right)^{r}}{n} \stackrel{q . s .}{\rightarrow} 0$.

Note que se $X_{0}=i$ então $\tau_{1}(i)$ tem a mesma distribuição que $\tau_{2}(i)-\tau_{1}(i)$ e, assim, $\frac{\left(\tau_{1}(i)\right)^{r}}{n} \stackrel{q . c .}{\rightarrow} 0$. Se $X_{0}=j \neq i$, então $\tau_{1}(i)$ representa a primeira visita ao estado $i$. Como a cadeia é recorrente, ao considerarmos o tempo passado, ela teria visitado o estado $i$ antes do tempo 0 . Segue que $E\left\{\tau_{1}(i)\right\} \leq E\left\{\tau_{2}(i)-\tau_{1}(i)\right\}$. Claramente, também temos que $E\left\{\left(\tau_{1}(i)\right)^{r}\right\}<\infty$ e o mesmo raciocínio acima nos leva a $\frac{\left(\tau_{1}(i)\right)^{r}}{n} \stackrel{\text { q.c. }}{\rightarrow} 0$.

(c) Como $Y_{k}(i)$ é $\sigma\left(X_{\tau_{k}(i)}, X_{\tau_{k}(i)+1}, \ldots, X_{\tau_{k+1}(i)-1}\right)$-mensurável, pelo item (c) da Proposição 1.1 temos que $Y_{1}(i), Y_{2}(i), \ldots$, são variáveis aleatórias i.i.d. . Além disso, não é necessário assumir que a independência é sobre $P_{i}$, pois estamos tomando $k \geq 1$. Para provarmos (2.7), note que 
$E\left\{\sum_{l=\tau_{k}(i)}^{\tau_{k+1}(i)-1} I\left(X_{l}=j\right)\right\}$ representa o número esperado de visitas ao estado $j$ entre visitas sucessivas ao estado $i$. Como $i$ e $j$ pertencem à mesma classe de recorrência, podemos usar a Proposição 1.2 ,

$$
\lim _{n \rightarrow \infty} \frac{\sum_{m=0}^{n} P_{i j}{ }^{m}}{\sum_{m=0}^{n} P_{i i}{ }^{m}}=\lim _{n \rightarrow \infty} \frac{\frac{1}{n} \sum_{m=0}^{n} P_{i j}{ }^{m}}{\frac{1}{n} \sum_{m=0}^{n} P_{i i}{ }^{m}}=\frac{\pi_{j}}{\pi_{i}} .
$$

Como $E_{\pi}\left\{\left|\phi\left(X_{0}\right)\right|\right\}<\infty$, podemos escrever

$$
\begin{aligned}
E\left\{\sum_{l=\tau_{k}}^{\tau_{k+1}-1} \phi\left(X_{l}\right)\right\} & =\sum_{j \in S} \phi(j) E\left\{\sum_{l=\tau_{k}}^{\tau_{k+1}-1} I\left(X_{l}=j\right)\right\} \\
& =\sum_{j \in S} \phi(j) \frac{\pi_{j}}{\pi_{i}}=\frac{1}{\pi_{i}} E_{\pi}\left\{\phi\left(X_{0}\right)\right\}=\frac{1}{\pi_{i}} \mu_{\phi}
\end{aligned}
$$

Por (2.3),

$$
E\left\{Y_{k}(i)\right\}=E\left\{\sum_{l=\tau_{k}}^{\tau_{k+1}-1}\left(\phi\left(X_{l}\right)-\mu_{\phi}\right)\right\}=\frac{\mu_{\phi}}{\pi_{i}}-\mu_{\phi} E\left\{\tau_{k+1}-\tau_{k}\right\}=0
$$

Exatamente os mesmos argumentos mostram que $E\left\{\sum_{l=\tau_{k}}^{\tau_{k+1}-1} \Psi\left(X_{l}\right)\right\}=\frac{1}{\pi_{i}} E_{\pi}\left\{\Psi\left(X_{0}\right)\right\}$.

A seguir, abordamos uma extensão da desigualdade (1.15) para números reais e alguns resultados relacionados à distância Mallows.

Lema 2.2 Para números reais $x_{1}, x_{2}, \ldots, x_{n}$ e $r \geq 1$, temos que

$$
\left|x_{1}+\ldots+x_{n}\right|^{r} \leq n^{r-1} \sum_{k=1}^{n}\left|x_{k}\right|^{r}
$$

Demonstração. Como $|x|^{r}$ é uma função convexa, temos que

$$
\left|\frac{x_{1}+x_{2}}{2}\right|^{r} \leq \frac{1}{2}\left(\left|x_{1}\right|^{r}+\left|x_{2}\right|^{r}\right) \leq 2^{r-1}\left(\left|x_{1}\right|^{r}+\left|x_{2}\right|^{r}\right) .
$$


Assuma que isso é verdade para todo $k \leq n$. Então para $n+1$ existem dois casos a analisar. Se $n+1$ é par então, por convexidade,

$$
\begin{aligned}
\left|\frac{x_{1}+\ldots+x_{n+1}}{2}\right|^{r} & \leq \frac{1}{2}\left(\left|x_{1}+\ldots+\frac{x_{n+1}}{2}\right|^{r}+\left|x_{\frac{n+1}{2}+1}+\ldots+x_{n+1}\right|\right) \\
& \leq \frac{1}{2}\left(\frac{n+1}{2}\right)^{r-1} \sum_{k=1}^{n+1}\left|x_{k}\right|^{r}=\frac{(n+1)^{r-1}}{2^{r}} \sum_{k=1}^{n+1}\left|x_{k}\right|^{r},
\end{aligned}
$$

por hipótese de indução. Assim,

$$
\left|x_{1}+\ldots+x_{n+1}\right|^{r} \leq(n+1)^{r-1} \sum_{k=1}^{n+1}\left|x_{k}\right|^{r} .
$$

Se $n+1$ é ímpar e $n \geq 3$, então por hipótese de indução

$$
\left|x_{1}+\ldots+x_{\frac{n}{2}+1}\right|^{r} \leq\left(\frac{n}{2}+1\right)^{r-1}\left(\left|x_{1}\right|^{r}+\ldots+\left|x_{\frac{n}{2}+1}\right|^{r}\right)
$$

$\mathrm{e}$

$$
\left|x_{\frac{n}{2}+2}+\ldots+x_{n+1}\right|^{r} \leq\left(\frac{n}{2}\right)^{r-1}\left(\left|x_{\frac{n}{2}+2}\right|^{r}+\ldots+\left|x_{n+1}\right|^{r}\right) .
$$

De outra maneira,

$$
\left|x_{1}+\ldots+x_{n+1}\right|^{r}=(n+1)^{r}\left|\frac{\frac{n}{2}+1}{n+1}\left(\frac{x_{1}+\ldots+x_{\frac{n}{2}+1}}{\frac{n}{2}+1}\right)+\frac{\frac{n}{2}}{n+1}\left(\frac{x_{\frac{n}{2}+2}+\ldots+x_{n+1}}{\frac{n}{2}}\right)\right|^{r},
$$

e, por convexidade,

$$
\left|x_{1}+\ldots+x_{n+1}\right|^{r} \leq(n+1)^{r}\left(\frac{\frac{n}{2}+1}{n+1}\right)\left|\frac{x_{1}+\ldots+x_{\frac{n}{2}+1}}{\frac{n}{2}+1}\right|^{r}+(n+1)^{r}\left(\frac{\frac{n}{2}}{n+1}\right)\left|\frac{x_{\frac{n}{2}+2}+\ldots+x_{n+1}}{\frac{n}{2}}\right|^{r},
$$

Agora, usando (2.8) e (2.9), obtemos

$$
\left|x_{1}+\ldots+x_{n+1}\right|^{r} \leq(n+1)^{r-1} \sum_{k=1}^{n+1}\left|x_{k}\right|^{r} .
$$

Lema 2.3 Seja $X$ uma cadeia de Markov aperiódica e recorrente positiva e assuma que para algum $i \in S$, algum $k \geq 1$ e $r>0$, temos $d_{r}\left(Y_{k}(i), Z\right)<\infty$, onde $Z$ possui uma distribuição $\alpha$-estável, com $1<\alpha<2$. Então, temos 
a) Se $1<\alpha<2$, nós necessariamente temos $r \leq \alpha e$

$$
E\left\{\left|Y_{k}(i)\right|^{r^{\prime}}\right\}<\infty, \quad 0<r^{\prime}<r
$$

b) Se $r \geq 2$, nós necessariamente temos o caso Gaussiano, $\alpha=2$. E, se $E\left\{\left(Y_{k}(i)\right)^{2}\right\}>0$, então

$$
d_{r}\left(\frac{Y_{k}(i)}{\sigma_{\phi}(i)}, Z_{0}\right)<\infty, \quad Z_{0} \stackrel{D}{=} \mathcal{N}(0,1), \sigma_{\phi}^{2}(i)=E\left\{\left(Y_{k}(i)\right)^{2}\right\}
$$

Além disso, temos $E\left\{\left|Y_{k}(j)\right|^{r}\right\}<\infty$, para todo $j \in S$.

c) Para todo $k^{\prime} \geq 1$, temos que $d_{r}\left(Y_{k^{\prime}}(i), Z\right)<\infty$. Além disso, para $0<r^{\prime}<r$, nós temos $d_{r^{\prime}}\left(Y_{l}(j), Z\right)<\infty$, para todo $j \in S$ e $l \geq 1$

Demonstração. (a) Como $Z$ é $\alpha$-estável, pela item (a) da Proposição 1.3, temos $E\left\{|Z|^{\alpha^{\prime}}\right\}<\infty$ para $0<\alpha^{\prime}<\alpha$ e $E\left\{|Z|^{\alpha}\right\}=\infty$. Assim, não podemos ter $r>\alpha$. Por outro lado, por Minkowsky,

$$
\left(E\left\{\left|Y_{k}(i)\right|^{r^{\prime}}\right\}\right)^{\frac{1}{r^{\prime}}} \leq d_{r^{\prime}}\left(Y_{k}(i), Z^{*}\right)+\left(E\left\{\left|Z^{*}\right|^{r^{\prime}}\right\}\right)^{\frac{1}{r^{\prime}}}
$$

onde consideramos $Z^{*}$ com uma distribuição $\alpha$-estável, $Z^{*} \stackrel{D}{=} Z$ e, além disso, $\left(Y_{k}(i), Z^{*}\right) \stackrel{D}{=} H$ com $H(x, y)=P\left(Y_{k}(i) \leq x\right) \wedge P(Z \leq y)$ tal que, pelo Teorema de Representação 1.2 ,

$$
d_{r^{\prime}}^{r^{\prime}}\left(Y_{k}(i), Z^{*}\right)=E\left\{\left|Y_{k}(i)-Z^{*}\right|^{r^{\prime}}\right\}
$$

Resta mostrar que $d_{r^{\prime}}\left(Y_{k}(i), Z^{*}\right)<\infty$, mas isso segue pela desigualdade de Liapounov, pois temos $0<r^{\prime}<r$.

(b) Por (a), se $r \geq 2$ nós não podemos ter $\alpha<2$. Como para distribuições $\alpha$-estáveis são para $\alpha \leq 2$, necessariamente temos $\alpha=2$. Para $Z$ Gaussiana, nós temos $E\left\{|Z|^{r}\right\}<\infty$ e, considerando $Z^{*}$ como acima, temos pela desigualdade de Minkowsky

$$
\left(E\left\{\left|Y_{k}(i)\right|^{r}\right\}\right)^{\frac{1}{r}} \leq d_{r}\left(Y_{k}(i), Z^{*}\right)+\left(E\left\{\left|Z^{*}\right|^{r}\right\}\right)^{\frac{1}{r}}<\infty,
$$

Como $E\left\{\left|Y_{k}(i)\right|^{r}\right\}<\infty$ e $r \geq 2$, a desigualdade de Liapounov nos dá $\sigma_{\phi}^{2}(i)=E\left\{Y_{k}^{2}(i)\right\}<\infty$. Se $\sigma_{\phi}^{2}(i)>0$, então $E\left\{\frac{Y_{k}^{2}(i)}{\sigma_{\phi}^{2}(i)}\right\}=1$ e $E\left\{Z_{0}^{2}\right\}=1$. Claramente, temos que $d_{r}\left(\frac{Y_{k}(i)}{\sigma_{\phi}(i)}, Z_{0}\right)<\infty$.

Como a cadeia é recorrente positiva, temos pelo Teorema 4 do Capítulo 14 de Chung (1960), que $E\left\{\left|Y_{k}(j)\right|^{r}\right\}<\infty$ dado que $E\left\{\left|Y_{k}(i)\right|^{r}\right\}<\infty$ para algum $i$.

(c) Pelo item (c) do Lema 2.1, as variáveis aleatórias $Y_{1}(i), Y_{2}(i), \ldots$, têm a mesma distribuição e, pela definição de distância Mallows, temos $d_{r}\left(Y_{k}(i), Z\right)=d_{r}\left(Y_{k^{\prime}}(i), Z\right), \forall k \geq 1$. Por (2.10) e para 
$0<r^{\prime}<r$, temos que $E\left\{\left|Y_{k}(i)\right|^{r^{\prime}}\right\}<\infty$. Como $i$ e $j$ pertencem à mesma classe de recorrência, o Teorema 4 do Capítulo 14 de Chung(1960) nos fornece $E\left\{\left|Y_{k}(j)\right|^{r^{\prime}}\right\}<\infty$. Mas $Y_{1}(j), Y_{2}(j), \ldots$, têm a mesma distribuição e isso conclui a prova.

Lema 2.4 Seja $V_{n}=\sum_{k=1}^{n} Y_{k}(i)$ e assuma que para algum $1<r \leq 2$ temos $E\left\{\left|Y_{k}(i)\right|^{r}\right\}<\infty e$ $\frac{V_{n}}{n^{\frac{1}{r}}} \stackrel{d}{\rightarrow} V$. Seja $\left\{l_{n}\right\}_{n \geq 1}$ definido como em (2.2), então se a cadeia é aperiódica e recorrente positiva, temos que $\frac{V_{l_{n}}}{l_{n}^{\frac{1}{r}}} \stackrel{d}{\rightarrow} V$.

Demonstração. Mostraremos que a sequência de variáveis aleatórias indexadas $\left\{l_{n}\right\}_{n \geq 1}$ e $\left\{\frac{V_{n}}{n^{\frac{1}{r}}}\right\}_{n \geq 1}$ satisfazem as condições do Lema 1.4. Por (2.4), temos $\frac{l_{n}}{n} \stackrel{q . c .}{\rightarrow} \pi_{i}>0$. Resta verificar que a condição de mixing de Ascombe (1.20) é satisfeita, ou seja, dado $\epsilon>0$ existe $\delta(\epsilon)$ tal que, para $n$ grande,

$$
L_{n}(\epsilon)=P\left(\max _{|l-n| \leq \delta n}\left|\frac{V_{l}}{l^{\frac{1}{r}}}-\frac{V_{n}}{n^{\frac{1}{r}}}\right| \geq \epsilon\right) \leq \epsilon
$$

Note que

Segue que

$$
\max _{|l-n| \leq \delta n}\left|\frac{V_{l}}{l^{\frac{1}{r}}}-\frac{V_{n}}{n^{\frac{1}{r}}}\right| \leq \max _{|l-n| \leq \delta n}\left|\frac{V_{l}}{l^{\frac{1}{r}}}-\frac{V_{l}}{n^{\frac{1}{r}}}\right|+\max _{|l-n| \leq \delta n}\left|\frac{V_{l}}{n^{\frac{1}{r}}}-\frac{V_{n}}{n^{\frac{1}{r}}}\right|
$$

$$
\begin{aligned}
L_{n}(\epsilon) & \leq L_{n, 1}(\epsilon)+L_{n, 2}(\epsilon) \\
& =P\left(\max _{|l-n| \leq \delta n}\left|\frac{V_{l}}{l^{\frac{1}{r}}}-\frac{V_{l}}{n^{\frac{1}{r}}}\right| \geq \frac{\epsilon}{2}\right)+P\left(\max _{|l-n| \leq \delta n}\left|\frac{V_{l}}{n^{\frac{1}{r}}}-\frac{V_{n}}{n^{\frac{1}{r}}}\right| \geq \frac{\epsilon}{2}\right) .
\end{aligned}
$$

Assim, temos para $L_{n, 1}(\epsilon)$,

$$
L_{n, 1}(\epsilon)=P\left(\max _{|l-n| \leq \delta n}\left|V_{l}\left(\frac{n^{\frac{1}{r}}-l^{\frac{1}{r}}}{(n l)^{\frac{1}{r}}}\right)\right| \geq \frac{\epsilon}{2}\right) .
$$

Agora,

$$
\begin{aligned}
\frac{n^{\frac{1}{r}}-l^{\frac{1}{r}}}{(n l)^{\frac{1}{r}}} & \leq \frac{n^{\frac{1}{r}}-n^{\frac{1}{r}}(1-\delta)^{\frac{1}{r}}}{n^{\frac{1}{r}} n^{\frac{1}{r}}(1-\delta)^{\frac{1}{r}}}=\frac{1-(1-\delta)^{\frac{1}{r}}}{n^{\frac{1}{r}}(1-\delta)^{\frac{1}{r}}} \\
& \leq \frac{1}{n^{\frac{1}{r}}} \frac{\delta^{\frac{1}{r}}}{(1-\delta)^{\frac{1}{r}}} .
\end{aligned}
$$


As desigualdades acima valem desde que $1<r \leq 2$ e nós podemos tomar $0<\delta<1$. Segue que

$$
L_{n, 1}(\epsilon) \leq P\left(\max _{|l-n| \leq \delta n}\left|V_{l}\right| \geq \frac{\epsilon}{2} \frac{n^{\frac{1}{r}}(1-\delta)^{\frac{1}{r}}}{\delta^{\frac{1}{r}}}\right) .
$$

Como $Y_{1}(i), Y_{2}(i), \ldots$, são variáveis aleatórias i.i.d. com média zero, observamos que o processo $\left\{V_{n}, \sigma\left(Y_{1}(i), \ldots, Y_{n}(i)\right)\right\}_{n \geq 1}$ forma uma martingale. Desde que $r>1$, pelas desigualdades de martingales (1.18) e (1.17), existe uma constante $C(r)>0$ tal que

$$
\begin{aligned}
L_{n, 1}(\epsilon) & \leq\left(\frac{2}{\epsilon}\right)^{r} \frac{\delta}{n(1-\delta)} E\left\{\left|V_{n}\right|^{r}\right\} \\
& \leq C(r)\left(\frac{2}{\epsilon}\right)^{r} \frac{\delta}{n(1-\delta)} E\left\{\left(\sum_{k=1}^{n} Y_{k}^{2}(i)\right)^{\frac{r}{2}}\right\} .
\end{aligned}
$$

Como $\frac{r}{2} \leq 1$, nós temos pela desigualdade (1.14)

$$
L_{n, 1}(\epsilon) \leq C(r)\left(\frac{2}{\epsilon}\right)^{r} \frac{\delta}{n(1-\delta)} n \cdot E\left\{\left|Y_{k}(i)\right|^{r}\right\}
$$

Como $E\left\{\left|Y_{k}(i)\right|^{r}\right\}<\infty$ por hipótese, tomando $0<\delta<\frac{\epsilon^{r+1}}{\epsilon^{r+1}+2^{r} C(r) E\left\{\left|Y_{k}(i)\right|^{r}\right\}}$, obtemos que $L_{n, 1}(\epsilon) \leq \frac{\epsilon}{2}$.

Para estimar o segundo termo $L_{n, 2}(\epsilon)$, denotemos [.] o maior inteiro e definamos $n_{1}=[n(1-\delta)] \mathrm{e}$ $n_{2}=[n(1+\delta)]$. Assim, note que

$$
\max _{|l-n| \leq \delta n}\left|\frac{V_{l}-V_{n}}{n^{\frac{1}{r}}}\right| \leq 2 \max _{n_{1} \leq m \leq n_{2}}\left|\frac{V_{m}-V_{n_{1}}}{n^{\frac{1}{r}}}\right|
$$

e que $\left\{V_{m}-V_{n_{1}}\right\}_{m \geq n_{1}+1}$ é também uma martingale. Assim, nós podemos proceder como acima, tal que para alguma constante $C^{\prime}(r)>0$ obtemos

$$
\begin{aligned}
L_{n, 2}(\epsilon) & \leq P\left(\max _{n_{1} \leq m \leq n_{2}}\left|V_{m}-V_{n_{1}}\right| \geq \frac{\epsilon}{4} n^{\frac{1}{r}}\right) \\
& \leq C^{\prime}(r)\left(\frac{4}{\epsilon}\right)^{r} \frac{1}{n} E\left\{\left(\sum_{k=n_{1}+1}^{n_{2}} Y_{k}^{2}(i)\right)^{\frac{r}{2}}\right\} \\
& \leq C^{\prime}(r)\left(\frac{4}{\epsilon}\right)^{r} \frac{n_{2}-n_{1}}{n} E\left\{\left|Y_{k}(i)\right|^{r}\right\} .
\end{aligned}
$$


Ao notarmos que $\frac{n_{2}-n_{1}}{n} \approx 2 \delta$, novamente para uma escolha adequada de $\delta$, obtemos $L_{n, 2}(\epsilon) \leq \frac{\epsilon}{2}$ e isso completa a prova.

\subsection{O Caso Gaussiano}

Considere a decomposição da soma parcial $S_{n}-n \mu_{\phi}, \operatorname{com} \mu_{\phi}=E_{\pi}\left\{\phi\left(X_{k}\right)\right\}, 0 \leq k \leq n$, dada por

$$
S_{n}-n \mu_{\phi}=A_{n}(i)+\sum_{k=1}^{l_{n}-1} Y_{k}(i)+B_{n}(i)
$$

com

$$
A_{n}(i)=\sum_{l=0}^{\tau_{1}(i)-1}\left(\phi\left(X_{l}\right)-\mu_{\phi}\right), B_{n}(i)=\sum_{l=\tau_{l n}(i)}^{n}\left(\phi\left(X_{l}\right)-\mu_{\phi}\right)
$$

$\mathrm{e}$

$$
Y_{k}(i)=\sum_{l=\tau_{k}(i)}^{\tau_{k+1}-1(i)}\left(\phi\left(X_{l}\right)-\mu_{\phi}\right)
$$

Nesta seção, para um estado $i \in S$ arbitrariamente escolhido, nós analisamos a normalidade assintótica de $S_{n}$ quando $0<\operatorname{var}\left\{Y_{k}(i)\right\}<\infty$. Mostraremos que, comparado ao termo central, os termos $A_{n}(i)$ e $B_{n}(i)$ são negligíveis. Assim, precisamos primeiro estabelecer condições que garantam a normalidade assintótica de $\sum_{k=1}^{l_{n}-1} Y_{k}(i)$. Nosso Lema 2.3 sugere a seguinte condição.

Condição 2.1 Seja $X=\left\{X_{n}\right\}_{n \geq 0}$ uma cadeia de Markov aperiódica e recorrente positiva. Assuma que para algum $i \in S$ e $k \geq 1$, temos $E\left\{Y_{k}^{2}(i)\right\}>0$ e que, para algum $r \geq 2$, temos que $d_{r}\left(Y_{k}(i), Z\right)<\infty$, onde $Z$ tem uma distribuição normal.

Note que, se a Condição 2.1 é satisfeita, nós temos $E\left\{\left|Y_{k}(i)\right|^{r}\right\}<\infty$ pelo item (b) do Lema 2.3. Então $E\left\{\left|Y_{k}(j)\right|^{r}\right\}<\infty$ para todo $j \in S$. Assim, a Condição 2.1 estaria satisfeita para todo $j \in S$. Como $r \geq 2$, nós também obtemos $0<E\left\{Y_{k}{ }^{2}(i)\right\}<\infty$ e, se trocarmos $\Psi($. ) no item (c) do Lema 2.1, por $\left(\phi(.)-\mu_{\phi}\right)^{2}$, obtemos

$$
E\left\{\sum_{l=\tau_{k}}^{\tau_{k+1}-1}\left(\phi\left(X_{l}\right)-\mu_{\phi}\right)^{2}\right\}=\frac{1}{\pi_{i}} E_{\pi}\left\{\left(\phi\left(X_{0}\right)-\mu_{\phi}\right)^{2}\right\}
$$


e, assim,

$$
\sigma_{\phi}^{2}(i)=\frac{1}{\pi_{i}} E_{\pi}\left\{\left(\phi\left(X_{0}\right)-\mu_{\phi}\right)^{2}\right\}+E\left\{\sum_{l \neq l^{\prime}=\tau_{k}}^{\tau_{k+1}-1}\left(\phi\left(X_{l}\right)-\mu_{\phi}\right)\left(\phi\left(X_{l^{\prime}}\right)-\mu_{\phi}\right)\right\} .
$$

Como mostrado no Capítulo 16 de Chung (1960), se ${\sigma_{\phi}}^{2}(i)<\infty$ para algum $i$, então ${\sigma_{\phi}}^{2}(j)<\infty$ para todo $j \in S$. Além disso, $\sigma_{\phi}^{2}=\pi_{i} \sigma_{\phi}^{2}(i)$ independe do estado $i$.

É importante destacar que para a normalidade assintótica necessitamos somente da Condição 2.1 satisfeita para $r=2$. Nesse caso, podemos estabelecer uma forma simples dela.

Condição 2.2 Seja X uma cadeia de Markov aperiódica e recorrente positiva, e assuma que para algum $i \in S$ e algum $k \geq 1$, temos que $0<E\left\{Y_{k}^{2}(i)\right\}<\infty$.

Não é difícil ver que se a Condição 2.2 é satisfeita, então

$$
d_{2}^{2}\left(Y_{k}(i), Z\right) \leq E\left\{\left(Y_{k}(i)-Z\right)^{2}\right\} \leq 2\left\{E\left\{Y_{k}^{2}(i)\right\}+E\left\{Z^{2}\right\}\right\}<\infty,
$$

e, assim, a Condição 2.1 vale para $r=2$.

Teorema 2.1 Assuma que a Condição 2.1 vale e seja $Z_{0} \stackrel{d}{=} \mathcal{N}(0,1)$. Então

$$
d_{r}\left(\frac{\sum_{k=1}^{n} Y_{k}(i)}{\sigma_{\phi}(i) \sqrt{n}}, Z_{0}\right) \stackrel{n \rightarrow \infty}{\longrightarrow} 0 .
$$

Além disso,

$$
\frac{\sum_{k=1}^{n} Y_{k}(i)}{\sigma_{\phi}(i) \sqrt{n}} \stackrel{d}{\longrightarrow} Z_{0} \quad e \quad E\left\{\left|\frac{\sum_{k=1}^{n} Y_{k}(i)}{\sigma_{\phi}(i) \sqrt{n}}\right|^{r}\right\} \stackrel{n \rightarrow \infty}{\longrightarrow} E\left\{\left|Z_{0}\right|^{r}\right\} .
$$

Demonstração. Pelo item (c) do Lema 2.1, $Y_{1}(i), Y_{2}(i), \ldots$, são variáveis aleatórias i.i.d. . Pelas hipóteses e o item (b) do Lema 2.3, temos que $0<\operatorname{var}\left(Y_{k}(i)\right)<\infty$. Assim, uma aplicação direta do Teorema 1.3 nos dá (2.11). Para provarmos (2.12), precisamos verificar as hipóteses do Teorema 1.1 . Como $E\left\{\left|Z_{0}\right|^{r}\right\}<\infty$, resta mostrarmos que

$$
E\left\{\left|\frac{\sum_{k=1}^{n} Y_{k}(i)}{\sigma_{\phi}(i) \sqrt{n}}\right|^{r}\right\}<\infty .
$$


Pelo Lema 2.3 (b) temos que $E\left\{\left|Y_{k}(i)\right|^{r}\right\}<\infty$. Como $\left\{\sum_{k=1}^{n} Y_{k}(i), \sigma\left(Y_{1}(i), Y_{2}(i), \ldots, Y_{n}(i)\right)\right\}$ forma uma martingale, podemos aplicar as desigualdades (1.17) e (1.15). Assim, existe uma constante $C(r)>0$ tal que

$$
\begin{aligned}
\frac{1}{n^{\frac{r}{2}}} \frac{1}{\sigma_{\phi}^{r}(i)} E\left\{\left|\sum_{k=1}^{n} Y_{k}(i)\right|^{r}\right\} & \leq \frac{1}{n^{\frac{r}{2}}} \frac{C(r)}{\sigma_{\phi}^{r}(i)} E\left\{\left|\sum_{k=1}^{n} Y_{k}^{2}(i)\right|^{\frac{r}{2}}\right\} \\
& \leq \frac{1}{n^{\frac{r}{2}}} \frac{C(r)}{\sigma_{\phi}^{r}(i)} n^{\frac{r}{2}-1} n E\left\{\left|Y_{k}(i)\right|^{r}\right\} \\
& \leq \frac{C(r)}{\sigma_{\phi}^{r}(i)} E\left\{\left|Y_{k}(i)\right|^{r}\right\}<\infty,
\end{aligned}
$$

e, assim, (2.12) segue pelo Teorema 1.1.

Teorema 2.2 Assuma que a Condição 2.1 vale e seja $Z_{0} \stackrel{d}{=} \mathcal{N}(0,1)$. Então

$$
d_{r}\left(\frac{V_{l_{n}-1}}{\sigma_{\phi}(i) \sqrt{l_{n}}}, Z_{0}\right) \stackrel{n \rightarrow \infty}{\longrightarrow} 0, V_{n}=\sum_{k=1}^{n} Y_{k}(i)
$$

Além disso,

$$
\frac{V_{l_{n}-1}}{\sigma_{\phi}(i) \sqrt{l_{n}}} \stackrel{d}{\longrightarrow} Z_{0} \quad e \quad E\left\{\left|\frac{V_{l_{n}-1}}{\sigma_{\phi}(i) \sqrt{l_{n}}}\right|^{r}\right\} \stackrel{n \rightarrow \infty}{\longrightarrow} E\left\{\left|Z_{0}\right|^{r}\right\} .
$$

Demonstração. Sem perda de generalidade, nós podemos assumir que $\sigma_{\phi}^{2}(i)=1$. Pelo Teorema anterior, temos que $\frac{V_{n}}{\sqrt{n}} \stackrel{d}{\rightarrow} Z_{0}$. Como $\frac{l_{n}-1}{n} \stackrel{\text { q.c. }}{\rightarrow} \pi_{i}>0$, pelo Lema 2.4 segue que $\frac{V_{l_{n}-1}}{\sqrt{l_{n}-1}} \stackrel{d}{\rightarrow} Z_{0}$. Como $\frac{\sqrt{l_{n}-1}}{\sqrt{l_{n}}} \stackrel{\text { q.c. }}{\rightarrow} 1$, obtemos que $\frac{V_{l_{n}-1}}{\sqrt{l_{n}}} \stackrel{d}{\rightarrow} Z_{0}$. Nós mostraremos que $E\left\{\left|\frac{V_{l_{n}-1}}{\sqrt{l_{n}}}\right|^{r}\right\}<\infty, \forall n$, e que (2.14) vale. Assim, pelo Teorema 1.1 seguirá (2.13).

Antes de provarmos a convergência à direita em (2.14), mostraremos que

$$
E\left\{\left|\frac{V_{l_{n}-1}}{\sqrt{l_{n}}}\right|^{r}\right\} \leq K<\infty
$$

Note que

$$
\left(l_{n}=k\right)=\left(\tau_{k} \leq n, \tau_{k+1}>n\right)=\left(\tau_{l_{n}} \leq n, \tau_{l_{n}+1}>n\right),
$$

tal que $\left(l_{n}=k\right)$ é $\sigma\left(X_{\tau_{l_{n}}}, X_{\tau_{l_{n}+1}}, \ldots, X_{\tau_{l_{n}+2}-1}\right)$-mensurável. Por outro lado, observe que $V_{l_{n}-1}$ é $\sigma\left(X_{\tau_{1}}, X_{\tau_{1}+1}, \ldots, X_{\tau_{l_{n}}-1}\right)$-mensurável. Pela Proposição 1.1(c), temos que $l_{n}$ e $\left(Y_{1}(i), \ldots, Y_{l n-1}(i)\right)$ 
são independentes. Sendo $E\left\{Y_{k}(i)\right\}=0$, o processo $\left\{V_{l_{n}-1}, \sigma\left(Y_{1}(i), \ldots, Y_{l n-1}(i)\right)\right\}$ é também uma martingale. Procedendo como na prova do teorema anterior, existe uma constante $C(r)$ tal que

$$
E\left\{\left|V_{l_{n}-1}\right|^{r}\right\} \leq C(r) E\left\{\left(\sum_{k=1}^{l_{n}-1} Y_{k}^{2}(i)\right)^{\frac{r}{2}}\right\}
$$

ou, equivalentemente,

$$
\begin{aligned}
E\left\{\left|V_{l_{n}-1}\right|^{r} / l_{n}\right\} & \leq C(r) E\left\{\left(\sum_{k=1}^{l_{n}-1} Y_{k}^{2}(i)\right)^{\frac{r}{2}} / l_{n}\right\} \\
& \leq C(r)\left(l_{n}-1\right)^{\frac{r}{2}-1} E\left\{\sum_{k=1}^{l_{n}-1}\left|Y_{k}(i)\right|^{r} / l_{n}\right\} \\
& \leq C(r) l_{n}^{\frac{r}{2}-1} E\left\{\sum_{k=1}^{l_{n}-1}\left|Y_{k}(i)\right|^{r} / l_{n}\right\},
\end{aligned}
$$

onde para a última desigualdade aplicamos o Lema 2.2. Agora,

$$
\begin{aligned}
E\left\{\left|\frac{V_{l_{n}-1}}{\sqrt{l_{n}}}\right|^{r}\right\} & =E\left\{\frac{1}{l_{n}^{\frac{r}{2}}} E\left\{\left|V_{l_{n}-1}\right|^{r} / l_{n}\right\}\right\} \\
& \leq C(r) E\left\{\frac{l_{n}^{\frac{r}{2}-1}}{l_{n}^{\frac{r}{2}}} E\left\{\sum_{k=1}^{l_{n}-1}\left|Y_{k}(i)\right|^{r} / l_{n}\right\}\right\} \\
& =C(r) E\left\{\left|Y_{k}(i)\right|^{r}\right\} .
\end{aligned}
$$

Assim, obtemos (2.15), desde que $E\left\{\left|Y_{k}(i)\right|^{r}\right\}<\infty$, pelo Lema 2.3(b).

Resta mostrar que $E\left\{\left|\frac{V_{l_{n}}-1}{\sqrt{l_{n}}}\right|^{r}\right\} \stackrel{n \rightarrow \infty}{\longrightarrow} E\left\{\left|Z_{0}\right|^{r}\right\}$. Como $\frac{l_{n}}{n} \stackrel{q . c .}{\rightarrow} \pi_{i}>0$, dado $\epsilon>0$, defina $A_{n}(\epsilon)=\left(\left|\frac{l_{n}}{n}-\pi_{i}\right| \leq \epsilon\right)$. Então, $P\left(A_{n}{ }^{c}(\epsilon)\right) \stackrel{n \rightarrow \infty}{\longrightarrow} 0$, conforme $\epsilon \rightarrow 0$. Por (2.15), temos que $\int_{A_{n}{ }^{c}(\epsilon)}\left|\frac{V_{l_{n}-1}}{\sqrt{l_{n}}}\right|^{r} d P \stackrel{n \rightarrow \infty, \epsilon \rightarrow 0}{\longrightarrow} 0$. Agora, nós devemos mostrar que

$$
\int_{A_{n}(\epsilon)}\left|\frac{V_{l_{n}-1}}{\sqrt{l_{n}}}-\frac{V_{n}}{\sqrt{n}}\right|^{r} d P \stackrel{n \rightarrow \infty, \epsilon \rightarrow 0}{\longrightarrow} 0 .
$$

Assim, nós temos a convergência desejada, pois o teorema anterior nos dá $E\left\{\left|\frac{V_{n}}{\sqrt{n}}\right|^{r}\right\} \stackrel{n \rightarrow \infty}{\longrightarrow} E\left\{\left|Z_{0}\right|^{r}\right\}$.

A prova de (2.16) é similar à prova do Lema 2.4 e nós daremos um resumo. Assim, definindo $n_{1}=\left[\left(\pi_{i}-\epsilon\right) n\right]$ e $n_{2}=\left[\left(\pi_{i}+\epsilon\right) n\right]$, temos que 


$$
\max _{n_{1} \leq l_{n} \leq n_{2}}\left|\frac{V_{l_{n}-1}}{\sqrt{l_{n}}}-\frac{V_{n}}{\sqrt{n}}\right| \leq \max _{n_{1} \leq l_{n} \leq n_{2}}\left|\frac{V_{l_{n}-1}}{\sqrt{n_{1}}}-\frac{V_{l_{n}-1}}{\sqrt{n_{2}}}\right|+\max _{n_{1} \leq m \leq n_{2}}\left|\frac{V_{m}}{\sqrt{n_{1}}}-\frac{V_{n_{1}}}{\sqrt{n_{1}}}\right| .
$$

Claramente, o mesmo vale com as correspondentes esperanças. Além disso, os processos $\left\{V_{l_{n}-1}\right\}$ e $\left\{V_{m}-V_{n_{1}}\right\}$ são martingales. Assim, usando a desigualdade de martingale (1.19), com $p=r$ e $q=\frac{r}{r-1}$, temos que

$$
\begin{aligned}
L_{n, 1}(\epsilon) & =E\left\{\max _{n_{1} \leq l_{n} \leq n_{2}}\left|V_{l_{n}-1}\right|^{r}\left(\frac{1}{\sqrt{n_{1}}}-\frac{1}{\sqrt{n_{2}}}\right)^{r}\right\} \\
& \leq\left(\frac{1}{\sqrt{n_{1}}}-\frac{1}{\sqrt{n_{2}}}\right)^{r} \frac{r}{r-1} E\left\{\left|V_{l_{n}-1}\right|^{r}\right\} \\
& =\left(1-\sqrt{\frac{n_{1}}{n_{2}}}\right)^{r} \frac{r}{r-1} E\left\{\left|\frac{V_{l_{n}-1}}{\sqrt{n_{1}}}\right|^{r}\right\} .
\end{aligned}
$$

Como $E\left\{\left|\frac{V_{l_{n}-1}}{\sqrt{n_{1}}}\right|^{r}\right\}<\infty$ por (2.15) e $\frac{n_{1}}{n_{2}} \approx \frac{\pi_{i}-\epsilon}{\pi_{i}+\epsilon}$, obtemos que $L_{n, 1}(\epsilon) \rightarrow 0$, conforme $n \rightarrow \infty$ e $\epsilon \rightarrow 0$. Além disso,

$$
\begin{aligned}
L_{n, 2}(\epsilon) & =E\left\{\max _{n_{1} \leq m \leq n_{2}}\left|\frac{V_{m}-V_{n_{1}}}{\sqrt{n_{1}}}\right|^{r}\right\} \\
& \leq \frac{1}{n_{1} \frac{r}{2}} \frac{r}{r-1} E\left\{\left|V_{m}-V_{n_{1}}\right|^{r}\right\} .
\end{aligned}
$$

As desigualdades (1.17) e (1.15) nos dão a existência de $C(r)>0$, tal que

$$
\begin{aligned}
E\left\{\left|V_{m}-V_{n_{1}}\right|^{r}\right\} & \leq C(r) E\left\{\left(\sum_{k=n_{1}}^{n_{2}} Y_{k}^{2}(i)\right)^{\frac{r}{2}}\right\} \\
& \leq C(r)\left(n_{2}-n_{1}+1\right)^{\frac{r}{2}-1} E\left\{\sum_{k=n_{1}}^{n_{2}}\left|Y_{k}(i)\right|^{r}\right\} \\
& =C(r)\left(n_{2}-n_{1}+1\right)^{\frac{r}{2}} E\left\{\left|Y_{k}(i)\right|^{r}\right\} .
\end{aligned}
$$

Segue que

$$
L_{n, 2}(\epsilon) \leq C(r) \frac{r}{r-1}\left(\frac{n_{2}-n_{1}+1}{n_{1}}\right)^{\frac{r}{2}} E\left\{\left|Y_{k}(i)\right|^{r}\right\}
$$

$\operatorname{Mas} \frac{n_{2}-n_{1}}{n_{1}} \approx \frac{2 \epsilon}{1-\epsilon} \mathrm{e} \frac{1}{n_{1}} \stackrel{n \rightarrow \infty}{\longrightarrow} 0$. Assim, $L_{n, 2}(\epsilon) \stackrel{n \rightarrow \infty, \epsilon \rightarrow 0}{\longrightarrow} 0$. 
Finalmente,

$$
\int_{A_{n}(\epsilon)}\left|\frac{V_{l_{n}-1}}{\sqrt{l_{n}}}-\frac{V_{n}}{\sqrt{n}}\right|^{r} d P \leq E\left\{\max _{n_{1} \leq l_{n} \leq n_{2}}\left|\frac{V_{l_{n}-1}}{\sqrt{l_{n}}}-\frac{V_{n}}{\sqrt{n}}\right|^{r}\right\}
$$

e (2.16) segue.

Nós podemos facilmente reescrever este teorema ao trocar $l_{n}$ por $\tau_{l_{n}}=\tau_{l_{n}}(i)$. Neste caso, é necessário substituir $\sigma_{\phi}{ }^{2}(i)$ por ${\sigma_{\phi}}^{2}=\pi_{i} \sigma_{\phi}{ }^{2}(i)$.

Teorema 2.3 Assuma que a Condição 2.1 vale e que $Z_{0} \stackrel{d}{=} \mathcal{N}(0,1)$. Então, para todo $i \in S$, temos

$$
d_{r}\left(\frac{\widetilde{V}_{\tau_{l_{n}-1}}}{\sigma_{\phi} \sqrt{\tau_{l_{n}}}}, Z_{0}\right) \stackrel{n \rightarrow \infty}{\longrightarrow} 0
$$

onde $\widetilde{V}_{\tau_{l_{n}}-1}=\sum_{l=\tau_{1}(i)}^{\tau_{l_{n}}(i)-1}\left(\phi\left(X_{l}\right)-\mu_{\phi}\right)$. Além disso,

$$
\frac{\widetilde{V}_{\tau_{l_{n}-1}}}{\sigma_{\phi} \sqrt{\tau_{l_{n}}}} \stackrel{d}{\longrightarrow} Z_{0} \quad e \quad E\left\{\left|\frac{\widetilde{V}_{\tau_{l_{n}-1}}}{\sigma_{\phi} \sqrt{\tau_{l_{n}}}}\right|^{r}\right\} \stackrel{n \rightarrow \infty}{\longrightarrow} E\left\{\left|Z_{0}\right|^{r}\right\}
$$

Demonstração. Pelo Lema 2.3(b), se a Condição 2.1 está satisfeita para algum $i \in S$, então ela está satisfeita para todo $j \in S$. Nós mostraremos que $E\left\{\left|\frac{\widetilde{V}_{\tau_{l_{n}-1}}}{\sigma_{\phi} \sqrt{\tau_{l_{n}}}}\right|^{r}\right\}<\infty$ e que (2.17) vale. Então pelo Teorema 1.1 nós teremos (2.18).

Por (2.15), observe que temos $E\left\{\left|\frac{V_{l_{n}-1}}{\sigma_{\phi}(i) \sqrt{l_{n}}}\right|^{r}\right\} \leq K<\infty$. Claramente, $0<\frac{l_{n}}{\tau_{l_{n}}} \leq 1 \mathrm{e}$ $\widetilde{V}_{\tau_{l_{n}-1}}=V_{l_{n}-1}=\sum_{k=1}^{l_{n}-1} Y_{k}(i)$. Segue que

$$
E\left\{\left|\frac{\widetilde{V}_{\tau_{l_{n}-1}}}{\sigma_{\phi} \sqrt{\tau_{l_{n}}}}\right|^{r}\right\}=E\left\{\left|\sqrt{\frac{l_{n}}{\tau_{l_{n}}}} \frac{V_{l_{n}-1}}{\sqrt{\pi_{i}} \sigma_{\phi}(i) \sqrt{l_{n}}}\right|^{r}\right\} \leq \frac{1}{\pi_{i}^{\frac{r}{2}}} E\left\{\left|\frac{V_{l_{n}-1}}{\sigma_{\phi}(i) \sqrt{l_{n}}}\right|^{r}\right\} \leq \frac{1}{\pi_{i}^{\frac{r}{2}}} K<\infty .
$$

Assim, para (2.17) nós temos, pelo Teorema da Representação 1.2 , a existência de $Z_{0}{ }^{*} \stackrel{d}{=} \mathcal{N}(0,1)$ tal que

$$
d_{r}\left(\frac{V_{l_{n}-1}}{\sigma_{\phi}(i) \sqrt{l_{n}}}, Z_{0}\right)=\left(E\left\{\left|\frac{V_{l_{n}-1}}{\sigma_{\phi}(i) \sqrt{l_{n}}}-Z_{0}\right|^{r}\right\}\right)^{\frac{1}{r}}=\left\|\frac{V_{l_{n}-1}}{\sigma_{\phi}(i) \sqrt{l_{n}}}-Z_{0} *\right\|_{r} \stackrel{n \rightarrow \infty}{\longrightarrow} 0 .
$$


Pela desigualdade de Minkowsky,

$$
\left\|\frac{\tilde{V}_{\tau_{l_{n}-1}}}{\sigma_{\phi} \sqrt{\tau_{l_{n}}}}-Z_{0} *\right\|_{r} \leq\left\|\sqrt{\frac{l_{n}}{\tau_{l_{n}}}} \frac{1}{\sqrt{\pi_{i}}}\left(\frac{V_{l_{n}-1}}{\sigma_{\phi}(i) \sqrt{l_{n}}}-Z_{0}^{*}\right)\right\|_{r}+\left\|\left(\sqrt{\frac{l_{n}}{\tau_{l_{n}}}} \frac{1}{\sqrt{\pi_{i}}}-1\right) Z_{0} *\right\|_{r}
$$

Como $\frac{l_{n}}{\tau_{l_{n}}} \leq 1$, temos que

$$
\left\|\sqrt{\frac{l_{n}}{\tau_{l_{n}}}} \frac{1}{\sqrt{\pi_{i}}}\left(\frac{V_{l_{n}-1}}{\sigma_{\phi}(i) \sqrt{l_{n}}}-Z_{0}^{*}\right)\right\|_{r} \leq \frac{1}{\sqrt{\pi_{i}}}\left\|\frac{V_{l_{n}-1}}{\sigma_{\phi}(i) \sqrt{l_{n}}}-Z_{0} *\right\|_{r} \stackrel{n \rightarrow \infty}{\longrightarrow} 0 .
$$

Pelo Lema 2.1, temos $\sqrt{\frac{l_{n}}{\tau_{l_{n}}}} \frac{1}{\sqrt{\pi_{i}}} \stackrel{q \cdot c ., n \rightarrow \infty}{\longrightarrow} 1$. Além disso,

$$
\left|\left(\sqrt{\frac{l_{n}}{\tau_{l_{n}}}} \frac{1}{\sqrt{\pi_{i}}}-1\right) Z_{0}{ }^{*}\right| \leq 2\left(\frac{1}{\sqrt{\pi_{i}}}-1\right)\left|Z_{0}{ }^{*}\right| .
$$

Como $E\left\{\left|Z_{0}{ }^{*}\right|^{r}\right\}<\infty$, obtemos pelo Teorema da Convergência Dominada

$$
\left\|\left(\sqrt{\frac{l_{n}}{\tau_{l_{n}}}} \frac{1}{\sqrt{\pi_{i}}}-1\right) Z_{0} *\right\|_{r} \stackrel{n \rightarrow \infty}{\longrightarrow} 0
$$

Agora, (2.17) segue por (1.9),

$$
d_{r}\left(\frac{\widetilde{V}_{\tau_{l_{n}-1}}}{\sigma_{\phi} \sqrt{\tau_{l_{n}}}}, Z_{0}\right) \leq\left\|\frac{\widetilde{V}_{\tau_{l_{n}-1}}}{\sigma_{\phi} \sqrt{\tau_{l_{n}}}}-Z_{0} *\right\|_{r}
$$

Com a notação acima, temos que

$$
\frac{S_{n}-n \mu_{\phi}}{\sigma_{\phi} \sqrt{n}}=\frac{A_{n}(i)}{\sigma_{\phi} \sqrt{n}}+\frac{\widetilde{V}_{\tau_{l_{n}-1}}}{\sigma_{\phi} \sqrt{n}}+\frac{B_{n}(i)}{\sigma_{\phi} \sqrt{n}},
$$

onde

$$
A_{n}(i)=\sum_{l=0}^{\tau_{1}-1}\left(\phi\left(X_{l}\right)-\mu_{\phi}\right) \text { e } B_{n}(i)=\sum_{l=\tau_{l_{n}}}^{n}\left(\phi\left(X_{l}\right)-\mu_{\phi}\right) \text {. }
$$

Como, para $r=2$, a Condição 2.2 implica a Condição 2.1 e, pelo Lema $2.1, \frac{\tau_{l_{n}}}{n} \stackrel{\text { q.c. }}{\rightarrow} 1$, temos por (2.18) 


$$
\frac{\widetilde{V}_{\tau_{l_{n}-1}}}{\sigma_{\phi} \sqrt{n}}=\sqrt{\frac{\tau_{l_{n}}}{n}} \frac{\widetilde{V}_{\tau_{l_{n}-1}}}{\sigma_{\phi} \sqrt{\tau_{l_{n}}}} \stackrel{d}{\longrightarrow} Z_{0}
$$

Além disso, pelo Teorema 8 do Capítulo 14 de Chung (1960), temos $\frac{A_{n}(i)}{\sigma_{\phi} \sqrt{n}} \stackrel{p}{\longrightarrow} 0$ e $\frac{B_{n}(i)}{\sigma_{\phi} \sqrt{n}} \stackrel{p}{\longrightarrow} 0$. Isso nos permite estabelecer:

Corolário 2.1 Assuma que a Condição 2.2 vale e seja $Z_{0} \stackrel{d}{=} \mathcal{N}(0,1)$. Então

$$
\frac{S_{n}-n \mu_{\phi}}{\sigma_{\phi} \sqrt{n}} \stackrel{d}{\longrightarrow} Z_{0}
$$

De maneira a adicionarmos convergência $d_{2}$ em (2.20), precisaremos de afirmações adicionais sobre os momentos. Suponha $E_{\pi}\left\{\left(\phi\left(X_{k}\right)-\mu_{\phi}\right)^{2}\right\}<\infty$, para $0 \leq k \leq n$, ou, equivalentemente, $d_{2}\left(\phi\left(X_{k}\right), Z_{0}\right)<\infty$, onde $Z_{0} \stackrel{d}{=} \mathcal{N}(0,1)$. Assim, pelo Lema 2.2,

$$
\begin{aligned}
E\left\{\left(\frac{A_{n}(i)}{\sigma_{\phi} \sqrt{n}}\right)^{2}\right\} & =E\left\{\frac{\left(\sum_{l=0}^{\tau_{1}-1}\left(\phi\left(X_{l}\right)-\mu_{\phi}\right)\right)^{2}}{n \sigma_{\phi}^{2}}\right\} \\
& \leq E\left\{\frac{\tau_{1} \sum_{l=0}^{\tau_{1}-1}\left(\phi\left(X_{l}\right)-\mu_{\phi}\right)^{2}}{n \sigma_{\phi}^{2}}\right\} .
\end{aligned}
$$

Além disso, podemos observar que o evento $\left(\tau_{1}=k\right)$ é $\sigma\left(X_{\tau_{1}}, X_{\tau_{1}+1}, \ldots, X_{\tau_{2}-1}\right)$-mensurável e $\sum_{l=0}^{\tau_{1}-1}\left(\phi\left(X_{l}\right)-\mu_{\phi}\right)^{2}$ é $\sigma\left(X_{0}, X_{1}, \ldots, X_{\tau_{1}-1}\right)$-mensurável. Assim, a Proposição 1.1(c) nos garante que $\tau_{1}$ e $\sum_{l=0}^{\tau_{1}-1}\left(\phi\left(X_{l}\right)-\mu_{\phi}\right)^{2}$ são independentes sob $P_{i}$. Se tempos negativos são considerados, então para algum tempo $m \leq 0$ nós teríamos $X_{m}=i, \tau_{1} \leq \tau_{1}-m$ e $\sum_{l=0}^{\tau_{1}-1}\left(\phi\left(X_{l}\right)-\mu_{\phi}\right)^{2} \leq \sum_{l=m}^{\tau_{1}-1}\left(\phi\left(X_{l}\right)-\mu_{\phi}\right)^{2}$. Isso nos permite escrever 


$$
\begin{aligned}
E\left\{\left(\frac{A_{n}(i)}{\sigma_{\phi} \sqrt{n}}\right)^{2}\right\} & \leq E_{i}\left\{\frac{\tau_{1} \sum_{l=0}^{\tau_{1}-1}\left(\phi\left(X_{l}\right)-\mu_{\phi}\right)^{2}}{n \sigma_{\phi}^{2}}\right\} \\
& =\frac{1}{\sigma_{\phi}^{2}} E_{i}\left\{\frac{\tau_{1}}{n}\right\} E_{i}\left\{\sum_{l=0}^{\tau_{1}-1}\left(\phi\left(X_{l}\right)-\mu_{\phi}\right)^{2}\right\}
\end{aligned}
$$

Agora, $E_{i}\left\{\frac{\tau_{1}}{n}\right\}=\frac{1}{\pi_{i} n} \stackrel{n \rightarrow \infty}{\longrightarrow} 0$ e, pelo Lema 2.1(c) com $\Psi()=.\left(\phi()-.\mu_{\phi}\right)$, temos que

$$
E_{i}\left\{\sum_{l=0}^{\tau_{1}-1}\left(\phi\left(X_{l}\right)-\mu_{\phi}\right)^{2}\right\}=E\left\{\sum_{l=\tau_{k}}^{\tau_{k+1}-1}\left(\phi\left(X_{l}\right)-\mu_{\phi}\right)^{2}\right\}=\frac{1}{\pi_{i}} E_{\pi}\left\{\left(\phi\left(X_{0}\right)-\mu_{\phi}\right)^{2}\right\} .
$$

Assim,

$$
E\left\{\left(\frac{A_{n}(i)}{\sigma_{\phi} \sqrt{n}}\right)^{2}\right\} \stackrel{n \rightarrow \infty}{\longrightarrow} 0
$$

Similarmente, temos que

$$
\begin{aligned}
E\left\{\left(\frac{B_{n}(i)}{\sigma_{\phi} \sqrt{n}}\right)^{2}\right\} & \leq E\left\{\frac{n-\tau_{l_{n}}+1}{n \sigma_{\phi}^{2}} \sum_{l=\tau_{l_{n}}}^{n}\left(\phi\left(X_{l}\right)-\mu_{\phi}\right)^{2}\right\} \\
& \leq E\left\{\frac{\tau_{l_{n}+1}-\tau_{l_{n}}}{n \sigma_{\phi}{ }^{2}} \sum_{l=\tau_{l_{n}}+1}\left(\phi\left(X_{l}\right)-\mu_{\phi}\right)^{2}\right\} .
\end{aligned}
$$

Pela Proposição 1.1, notemos que $\left(\tau_{l_{n}+1}-\tau_{l_{n}}\right) \sum_{l=\tau_{l_{n}}}^{\tau_{l_{n}+1}-1}\left(\phi\left(X_{l}\right)-\mu_{\phi}\right)^{2}$ tem a mesma distribuição que $\tau_{1} \sum_{l=0}^{\tau_{1}-1}\left(\phi\left(X_{l}\right)-\mu_{\phi}\right)^{2}$ dado que $X_{0}=i$, ou seja, sob $P_{i}$. Segue que $E\left\{\left(\frac{B_{n}(i)}{\sigma_{\phi} \sqrt{n}}\right)^{2}\right\} \stackrel{n \rightarrow \infty}{\longrightarrow} 0$.

Logo, como

$$
d_{2}\left(\sqrt{\frac{\tau_{l_{n}}}{n}} \frac{\widetilde{V}_{\tau_{l_{n}}-1}}{\sigma_{\phi} \sqrt{\tau_{l_{n}}}}, Z_{0}\right) \stackrel{n \rightarrow \infty}{\longrightarrow} 0
$$

obtemos o seguinte corolário. 
Corolário 2.1 Assuma que a Condição 2.2 vale e que $E_{\pi}\left\{\left(\phi\left(X_{k}\right)-\mu_{\phi}\right)^{2}\right\}<\infty$, para $0 \leq k \leq n$. Então, para $Z_{0} \stackrel{d}{=} \mathcal{N}(0,1)$, temos que

$$
\begin{gathered}
d_{2}\left(\frac{S_{n}-n \mu_{\phi}}{\sigma_{\phi} \sqrt{n}}, Z_{0}\right) \stackrel{n \rightarrow \infty}{\longrightarrow} 0, \\
\frac{S_{n}-n \mu_{\phi}}{\sigma_{\phi} \sqrt{n}} \stackrel{d}{\longrightarrow} Z_{0} \quad e \quad E\left\{\left(\frac{S_{n}-n \mu_{\phi}}{\sigma_{\phi} \sqrt{n}}\right)^{2}\right\} \stackrel{n \rightarrow \infty}{\longrightarrow} E\left\{Z_{0}^{2}\right\}=1 .
\end{gathered}
$$

Observação 2.1 Como a Condição 2.2 requer $E\left\{Y_{k}^{2}(i)\right\}=E\left\{\left(\sum_{l=\tau_{k}}^{\tau_{k+1}-1}\left(\phi\left(X_{l}\right)-\mu_{\phi}\right)\right)^{2}\right\}<\infty$, nós conjeturamos que isso garantiria que $E_{\pi}\left\{\left(\phi\left(X_{0}\right)-\mu_{\phi}\right)^{2}\right\}<\infty$. Mas nós não estamos hábeis a mostrar isso.

Para generalizarmos o corolário acima para $r>2$, observe que o Lema 2.2 foi usado para obter desigualdades do tipo

$$
E_{i}\left\{\left|\frac{\sum_{l=0}^{\tau_{1}-1}\left(\phi\left(X_{l}\right)-\mu_{\phi}\right)}{\sqrt{n}}\right|^{r}\right\} \leq E_{i}\left\{\frac{\tau_{1}^{r-1}}{n^{\frac{r}{2}}} \sum_{l=0}^{\tau_{1}-1}\left|\phi\left(X_{l}\right)-\mu_{\phi}\right|^{r}\right\} .
$$

Isso sugere que além de assumirmos $E_{\pi}\left\{\left(\phi\left(X_{0}\right)-\mu_{\phi}\right)^{r}\right\}<\infty$, precisamos controlar $E_{i}\left\{\tau_{1}^{r-1}\right\}$. Sendo a cadeia recorrente positiva, temos que $E_{i}\left\{\tau_{1}\right\}<\infty$, que é o caso para $r=2$. No caso geral, para $r \geq 2$, sabendo que $\frac{\tau_{1}}{n} \leq 1$ e considerando $E_{i}\left(\tau_{1}^{\frac{r}{2}}\right)<\infty$, obtemos

$$
E_{i}\left\{\frac{\tau_{1}^{r-1}}{n^{\frac{r}{2}}}\right\}=E_{i}\left\{\left(\frac{\tau_{1}}{n}\right)^{\frac{r}{2}-1} \frac{\tau_{1}^{\frac{r}{2}}}{n}\right\} \leq \frac{E_{i}\left(\tau_{1}^{\frac{r}{2}}\right)}{n} \stackrel{n \rightarrow \infty}{\longrightarrow} 0 .
$$

Condição 2.3 Seja X uma cadeia de Markov aperiódica e recorrente positiva. Assuma que para algum $i \in S$ e $k \geq 1$ temos que $E\left\{Y_{k}^{2}(i)\right\}=\sigma_{\phi}^{2}(i)>0$. Além disso, assuma que para algum $r \geq 2$ temos que $E_{\pi}\left\{\left|\phi\left(X_{k}\right)-\mu_{\phi}\right|^{r}\right\}<\infty$, para $0 \leq k \leq n, E\left\{\left(\tau_{k+1}(i)-\tau_{k}(i)\right)^{\frac{r}{2}}\right\}<\infty e$ $d_{r}\left(Y_{k}(i), Z\right)<\infty$, onde $Z$ tem uma distribuição normal.

Assim como para a Condição 2.1, se a Condição 2.3 é satisfeita para algum $i \in S$ e algum $k \geq 1$, ela é satisfeita para todo $i \in S$ e todo $k \geq 1$. A hipótese $d_{r}\left(Y_{k}(i), Z\right)<\infty$ garante que $E\left\{\left|Y_{k}(i)\right|^{r}\right\}<\infty$ e o Lema 2.3(b) mostra que $d_{r}\left(\frac{Y_{k}(i)}{\sigma_{\phi}(i)}, Z_{0}\right)<\infty$, fornecido que $\sigma_{\phi}(i)>0, Z_{0} \stackrel{d}{=} \mathcal{N}(0,1)$. 
Teorema 2.4 Assuma que a Condição 2.3 vale. Então, para $\mu_{\phi}=E_{\pi}\left\{\phi\left(X_{k}\right)\right\}, 0 \leq k \leq n$, e $\sigma_{\phi}^{2}=\pi_{i} \sigma_{\phi}^{2}(i)$

$$
\begin{gathered}
d_{r}\left(\frac{S_{n}-n \mu_{\phi}}{\sigma_{\phi} \sqrt{n}}, Z_{0}\right) \stackrel{n \rightarrow \infty}{\longrightarrow} 0, \quad Z_{0} \stackrel{d}{=} \mathcal{N}(0,1), \\
\frac{S_{n}-n \mu_{\phi}}{\sigma_{\phi} \sqrt{n}} \stackrel{d}{\longrightarrow} Z_{0} \quad e \quad E\left\{\left|\frac{\mid S_{n}-n \mu_{\phi}}{\sigma_{\phi} \sqrt{n}}\right|^{r}\right\} \stackrel{n \rightarrow \infty}{\longrightarrow} E\left\{\left|Z_{0}\right|^{r}\right\} .
\end{gathered}
$$

Demonstração. Nós faremos uso do Teorema 1.1 ao mostrarmos que

$$
E\left\{\left|\frac{S_{n}-n \mu_{\phi}}{\sigma_{\phi} \sqrt{n}}\right|^{r}\right\}<\infty, \quad \forall n
$$

e que (2.22) vale. Consequentemente, teremos (2.23).

Usando a decomposição (2.19) e a notação do Teorema 2.17, temos pela desigualdade de Minkowsky,

$$
\left\|\frac{S_{n}-n \mu_{\phi}}{\sigma_{\phi} \sqrt{n}}\right\|_{r} \leq\left\|\frac{A_{n}(i)}{\sigma_{\phi} \sqrt{n}}\right\|_{r}+\left\|\frac{\widetilde{V}_{\tau_{l n}-1}}{\sigma_{\phi} \sqrt{n}}\right\|_{r}+\left\|\frac{B_{n}(i)}{\sigma_{\phi} \sqrt{n}}\right\|_{r} .
$$

Pelo Teorema 2.17 e o fato de que $\frac{\tau_{l_{n}}}{n} \leq 1$, obtemos

$$
E\left\{\left|\frac{\widetilde{V}_{\tau_{l n}-1}}{\sigma_{\phi} \sqrt{n}}\right|^{r}\right\}=E\left\{\left|\sqrt{\frac{\tau_{l_{n}}}{n}} \frac{\widetilde{V}_{\tau_{l n}-1}}{\sigma_{\phi} \sqrt{\tau_{l_{n}}}}\right|^{r}\right\} \leq E\left\{\left|\frac{\widetilde{V}_{\tau_{l n}-1}}{\sigma_{\phi} \sqrt{\tau_{l_{n}}}}\right|^{r}\right\}<\infty .
$$

Pelo Lema 2.3(b), temos que

$$
E_{i}\left\{\sum_{l=0}^{\tau_{1}-1}\left|\phi\left(X_{l}\right)-\mu_{\phi}\right|^{r}\right\}=\frac{1}{\pi_{i}} E_{\pi}\left\{\left|\phi\left(X_{0}\right)-\mu_{\phi}\right|^{r}\right\}<\infty .
$$

Como $E_{i}\left(\tau_{1}^{\frac{r}{2}}\right)=E_{i}\left\{\left(\tau_{k+1}-\tau_{k}\right)^{\frac{r}{2}}\right\}<\infty$, obtemos

$$
E\left\{\left|\frac{A_{n}(i)}{\sigma_{\phi} \sqrt{n}}\right|^{r}\right\} \stackrel{n \rightarrow \infty}{\longrightarrow} 0
$$

Analogamente, mostra-se que

$$
E\left\{\left|\frac{B_{n}(i)}{\sigma_{\phi} \sqrt{n}}\right|^{r}\right\} \stackrel{n \rightarrow \infty}{\longrightarrow} 0
$$

e, assim, (2.24) segue. Agora, o mesmo tipo de argumento como no caso $r=2$ nos dá (2.22). 


\subsection{O Caso Cauda-Pesada}

Nesta seção, lidamos com o caso onde a média é finita, $\mu_{\phi}=E_{\pi}\left\{\phi\left(X_{k}\right)\right\}<\infty, 0 \leq k \leq n$, mas o segundo momento não é finito, tal que nos deparamos com uma situação de cauda-pesada e os resultados para o caso Gaussiano não são aplicáveis. Como as distribuições $\alpha$-estáveis, $S_{\alpha}(\sigma, 0, \mu)$ com $1<\alpha<2$, estão relacionadas com a mesma lógica que a distribuição normal para somas parciais de variáveis aleatórias i.i.d., é natural conjeturar que, sob condições adequadas, o mesmo valerá para funcionais de cadeias de Markov. Para $X=\left\{X_{n}\right\}_{n \geq 0}$ e $\phi: S \rightarrow \mathbb{R}$, espera-se que a soma parcial estabilizada satisfaça

$$
\frac{S_{n}-n \mu_{\phi}}{n^{\frac{1}{\alpha}}}=\frac{\sum_{l=0}^{n}\left(\phi\left(X_{l}\right)-\mu_{\phi}\right)}{n^{\frac{1}{\alpha}}} \stackrel{d}{\longrightarrow} S_{\alpha}(\sigma, 0,0), \quad 1<\alpha<2 .
$$

Claramente, se nós tomarmos $S_{\alpha}(\sigma, 0, \mu)$ como a distribuição limite em (2.25), então o Teorema 1.4 mostra que teríamos

$$
\frac{S_{n}-n \mu_{\phi}+n^{\frac{1}{\alpha}} \mu}{n^{\frac{1}{\alpha}}} \stackrel{d}{\longrightarrow} S_{\alpha}(\sigma, 0, \mu) .
$$

Para ilustrar essa situação, nosso exemplo abaixo exibe um processo de excesso (residual) que possui uma distribuição limite de cauda-pesada.

Exemplo 2.1 Seja $X=\left\{X_{n}\right\}_{n \geq 0}$ uma cadeia de Markov irredutível, recorrente positiva, não-periódica e homogênea no tempo, com espaço de estados $\{1,2,3, \ldots\}$. Considere $P\left\{X_{1}=i-1 \mid X_{0}=i\right\}=1$, $\forall i \geq 2$, e denote por $F$ a distribuição do salto do estado 1, ou seja, para todo $i \in Z^{+}$,

$$
F(i)=P\left\{X_{1}=i+1 \mid X_{0}=1\right\} .
$$

Vamos mostrar que se $F$ é de cauda-pesada, ou seja, possui segundo momento infinito, então a distribuição limite de $X$ também é de cauda pesada. Para isso, inicialmente vamos exibir a matriz de transição de X, e encontrar sua distribuição limite. De fato, tal matriz é dada por

$$
\left(P_{i j}\right)=\left(\begin{array}{cccccc}
F(0) & F(1) & F(2) & F(3) & F(4) & \cdots \\
1 & 0 & 0 & 0 & 0 & \cdots \\
0 & 1 & 0 & 0 & 0 & \cdots \\
0 & 0 & 1 & 0 & 0 & \cdots \\
0 & 0 & 0 & 1 & 0 & \cdots \\
\vdots & \vdots & \ddots & \vdots & \vdots & \vdots \\
0 & 0 & 0 & 0 & 0 & \cdots
\end{array}\right) .
$$


Para o cálculo da distribuição estacionária, como $\pi P=\pi, \sum_{j=1}^{\infty} \pi_{j}=1$ e, pela matriz de transição $P, \sum_{i=0}^{\infty} F(i)=1$, obtemos

$$
\pi_{k}=\pi_{1}\left(1-\sum_{i=0}^{k-2} F(i)\right), \forall k \geq 2
$$

Agora, se chamarmos $h(k)=\sum_{i=k-1}^{\infty} F(i)=1-\sum_{i=0}^{k-2} F(i)$, temos que $\lim _{k \rightarrow \infty} h(k)=0$. Assim, existe $M \in \mathbb{N}$ tal que $h(k)=0$, para todo $k \geq M$. Disso, temos que $\pi_{1}=\frac{1}{L}$, onde

$$
L=\sum_{k=1}^{\infty}\left(1-\sum_{i=0}^{k-2} F(i)\right)=\sum_{k=1}^{M} h(k)<\infty .
$$

Portanto, $\pi=\left(\frac{1}{L}, \frac{h(2)}{L}, \frac{h(3)}{L}, \ldots\right)$.

Assumiremos, assim, que $F$ é de cauda-pesada, ou seja, $\sum_{k=0}^{\infty} k^{2} F(k)=\infty$, e mostraremos que

$$
\begin{aligned}
& \sum_{k=1}^{\infty}\left(k^{2} \frac{1-\sum_{i=0}^{k-2} F(i)}{L}\right)=\infty \text {. De fato, como } \\
& 1-\sum_{i=0}^{k-2} F(i)=F(k-1)+\sum_{i=k}^{\infty} F(i),
\end{aligned}
$$

obtemos que

$$
\sum_{k=1}^{\infty}\left(k^{2} \frac{1-\sum_{i=0}^{k-2} F(i)}{L}\right)=\frac{\sum_{k=1}^{\infty} k^{2} F(k-1)}{L}+\frac{\sum_{k=1}^{\infty} k^{2} \sum_{i=k}^{\infty} F(i)}{L}
$$

Assim, como $\lim _{k \rightarrow \infty} \sum_{i=k}^{\infty} F(i)=0$, existe $n \in \mathbb{N}$ suficientemente grande, tal que 


$$
\frac{\sum_{k=1}^{\infty} k^{2} \sum_{i=k}^{\infty} F(i)}{L}=\frac{\sum_{k=1}^{n} k^{2} \sum_{i=k}^{\infty} F(i)}{L} \in(0, \infty)
$$

Portanto, por (2.26),

$$
\begin{aligned}
\sum_{k=1}^{\infty} k^{2}\left(\frac{1-\sum_{i=0}^{k-2} F(i)}{L}\right) \geq & \frac{\sum_{k=1}^{\infty} k^{2} F(k-1)}{L} \\
& =\frac{\sum_{k=0}^{\infty}(k+1)^{2} F(k)}{L} \\
& \geq \frac{\sum_{k=0}^{\infty} k^{2} F(k)}{L}=\infty,
\end{aligned}
$$

e o exemplo está concluído.

Assim como no caso Gaussiano, nós consideraremos a decomposição (dissecção) conforme Chung (1960),

$$
S_{n}-n \mu_{\phi}=A_{n}(i)+\sum_{k=1}^{l_{n}-1} Y_{k}(i)+B_{n}(i), \quad Y_{k}(i)=\sum_{l=\tau_{k}(i)}^{\tau_{k+1}(i)-1}\left(\phi\left(X_{l}\right)-\mu_{\phi}\right) .
$$

Condição 2.4 Seja X uma cadeia de Markov aperiódica e recorrente positiva. Assuma que para algum $1<\alpha<2$ existe uma variável aleatória $\alpha$-estável $Z \stackrel{d}{=} S_{\alpha}\left(\sigma_{i}, 0,0\right)$ tal que $d_{\alpha}\left(Y_{k}(i), Z\right)<\infty$, para algum $i \in S$ e algum $k \geq 1$.

Note que pela Proposição 1.3 temos que $E\left\{|Z|^{\alpha}\right\}=\infty$ tal que o fato que $d_{\alpha}\left(Y_{k}(i), Z\right)<\infty$ necessariamente garante que $Y_{k}(i)$ não é uma constante. Assim, o requerimento do tipo $E\left\{Y_{k}^{2}(i)\right\}>0$ na Condição 2.1 é desnecessário para o caso $\alpha$-estável, $1<\alpha<2$. Além disso, se $d_{\alpha}\left(Y_{k}(i), Z\right)<\infty$ para alguma variável $\alpha$-estável não-enviesada $Z \stackrel{d}{=} S_{\alpha}\left(\sigma_{i}, 0, \mu\right)$, então temos que $d_{\alpha}\left(Y_{k}(i), Z^{\prime}\right)<\infty$ para $Z^{\prime} \stackrel{d}{=} S_{\alpha}\left(\sigma_{i}, 0,0\right)$. Isso nos permite reestabelecer a condição de uma forma aparentemente menos restritiva, mas equivalente: trocando $S_{\alpha}\left(\sigma_{i}, 0,0\right)$ por $S_{\alpha}\left(\sigma_{i}, 0, \mu\right)$. 
Pelo Lema 2.3(c), se a Condição 2.4 é satisfeita para algum estado $i$ e algum $k \geq 1$, então ela é satisfeita para todo $k \geq 1$. Além disso, como a cadeia é recorrente positiva, nós temos para todo $j \in S$, $d_{\alpha^{\prime}}\left(Y_{k}(j), Z\right)<\infty$ para $0<\alpha^{\prime}<\alpha$. Nossa Observação 2.2 adiante mostrará que se nós assumirmos adicionalmente que $E_{\pi}\left\{\left|\phi\left(X_{k}\right)-\mu_{\phi}\right|^{\alpha^{\prime}}\right\}<\infty$, para $0<\alpha^{\prime}<\alpha$, então se a Condição 2.4 é satisfeita para o estado $i$ com $Z \stackrel{d}{=} S_{\alpha}\left(\sigma_{i}, 0,0\right)$, e é também satisfeita para o estado $j$ com $Z \stackrel{d}{=} S_{\alpha}\left(\sigma_{j}, 0,0\right)$, então $\pi_{i}^{\frac{1}{\alpha}} \sigma_{i}=\pi_{j}^{\frac{1}{\alpha}} \sigma_{j}=\sigma$. De fato, mostraremos que

$$
\frac{S_{n}-n \mu_{\phi}}{n^{\frac{1}{\alpha}}} \stackrel{d}{\longrightarrow} Z \stackrel{d}{=} S_{\alpha}(\sigma, 0,0) .
$$

Teorema 2.5 Assuma que a Condição 2.4 é satisfeita para o estado i e seja $Z \stackrel{d}{=} S_{\alpha}\left(\sigma_{i}, 0,0\right)$ para algum $\sigma_{i}>0$. Então

$$
d_{\alpha}\left(\frac{\sum_{k=1}^{n} Y_{k}(i)}{n^{\frac{1}{\alpha}}}, Z\right) \stackrel{n \rightarrow \infty}{\longrightarrow} 0 .
$$

Além disso, para $1<\alpha^{\prime}<\alpha$, temos

$$
\frac{\sum_{k=1}^{n} Y_{k}(i)}{n^{\frac{1}{\alpha}}} \stackrel{d}{\longrightarrow} Z \quad e \quad E\left\{\left|\frac{\mid \sum_{k=1}^{n} Y_{k}(i)}{n^{\frac{1}{\alpha}}}\right|^{\alpha^{\prime}}\right\} \stackrel{n \rightarrow \infty}{\longrightarrow} E\left\{|Z|^{\alpha^{\prime}}\right\} .
$$

Note que, pela Proposição 1.3, temos $E\left\{|Z|^{\alpha}\right\}=\infty$ e, se (2.27) vale, então necessariamente nós temos $E\left\{\left|\frac{\sum_{k=1}^{n} Y_{k}(i)}{n^{\frac{1}{\alpha}}}\right|^{\alpha}\right\}=\infty$. Segue que em (2.28) nós não podemos esperar trocar $\alpha^{\prime}$ por $\alpha$ como no caso Gaussiano. Para provar (2.27), como $Y_{1}(i), Y_{2}(i), \ldots$, são variáveis aleatórias i.i.d. , é simples verificar que as condições do tipo Lindeberg do Teorema 1.4 são satisfeitas e a prova de (2.27) pode ser obtida como um caso particular do Teorema 1.4. Ao invés disso, daremos uma prova simples e direta baseada em integrabilidade uniforme e desigualdades de martingale, seguindo as idéias em Soares (2015). 
Demonstração. (Teorema 2.5)

(i) Seja $Z^{*} \stackrel{d}{=} Z \stackrel{d}{=} S_{\alpha}\left(\sigma_{i}, 0,0\right)$. Como $\alpha>1$ e $d_{\alpha}\left(Y_{k}(i), Z\right)=d_{\alpha}\left(Y_{k}(i), Z^{*}\right)$, pelo Teorema de Representação 1.2, podemos tomar $Z^{*}$ tal que a distribuição conjunta de $\left(Y_{k}(i), Z^{*}\right)$ é $F_{Y_{k}(i)} \wedge G_{Z^{*}}$, sendo $F_{Y_{k}(i)}$ e $G_{Z^{*}}$ as respectivas distribuições de $Y_{k}(i)$ e $Z^{*}$. Neste caso, temos que

$$
d_{\alpha}{ }^{\alpha}\left(Y_{k}(i), Z\right)=E\left\{\left|Y_{k}(i)-Z^{*}\right|^{\alpha}\right\}<\infty
$$

Pelo Lema 2.1(c), nós temos que $E\left\{Y_{k}(i)\right\}=0$ e $Y_{1}(i), Y_{2}(i), \ldots$, são variáveis aleatórias i.i.d. . Assim, podemos tomar $Z_{1}{ }^{*}, Z_{2}{ }^{*}, \ldots$, cópias independentes de $Z^{*}$ tais que

$$
d_{\alpha}{ }^{\alpha}\left(Y_{k}(i), Z\right)=E\left\{\left|Y_{k}(i)-Z_{k}{ }^{*}\right|^{\alpha}\right\}<\infty, \quad k \geq 1 .
$$

Além disso, pela Proposição 1.3, temos que

$$
\frac{Z_{1}^{*}+\ldots+Z_{n}^{*}}{n^{\frac{1}{\alpha}}} \stackrel{d}{=} Z^{*}
$$

Agora, $\left\{\sum_{k=1}^{n}\left(Y_{k}(i)-Z_{k}{ }^{*}\right), \sigma\left(Y_{1}(i)-Z_{1}{ }^{*}, Y_{2}(i)-Z_{2}{ }^{*}, \ldots, Y_{n}(i)-Z_{n}{ }^{*}\right)\right\}_{n \geq 1}$ forma uma martingale. Assim, pelo Lema 1.2, nós temos para $1<\alpha<2$,

$$
\frac{1}{n} E\left\{\left|\sum_{k=1}^{n}\left(Y_{k}(i)-Z_{k}^{*}\right)\right|^{\alpha}\right\} \stackrel{n \rightarrow \infty}{\longrightarrow} 0
$$

dado que $\left\{\left|Y_{k}(i)-Z_{k}\right|^{\alpha}\right\}_{k \geq 1}$ é uniformemente integrável. Como $Y_{1}(i), Y_{2}(i), \ldots$, são i.i.d. , nós temos para $c>0$,

$$
\sup _{m} \int_{\left(\left|Y_{m}(i)-Z_{m}{ }^{*}\right|>c\right)}\left|Y_{m}(i)-Z_{m}{ }^{*}\right|^{\alpha} d P=\int_{\left(\left|Y_{k}(i)-Z_{k}{ }^{*}\right|>c\right)}\left|Y_{k}(i)-Z_{k}{ }^{*}\right|^{\alpha} d P \stackrel{c \rightarrow \infty}{\longrightarrow} 0,
$$

onde a última convergência segue de (2.29) e, assim, obtemos a integrabilidade uniforme.

Para provarmos (2.27), nós temos, por (2.30),

$$
\begin{aligned}
d_{\alpha}{ }^{\alpha}\left(\frac{\sum_{k=1}^{n} Y_{k}(i)}{n^{\frac{1}{\alpha}}}, Z\right) & \leq E\left|\frac{\sum_{k=1}^{n} Y_{k}(i)}{n^{\frac{1}{\alpha}}}-\frac{\sum_{k=1}^{n} Z_{k}^{*}}{n^{\frac{1}{\alpha}}}\right|^{\alpha} \\
& \leq E\left\{\frac{1}{n}\left|\sum_{k=1}^{n}\left(Y_{k}(i)-Z_{k}^{*}\right)\right|^{\alpha}\right\},
\end{aligned}
$$


e o resultado segue por (2.31). Claramente, também temos a parte à esquerda de (2.28).

(ii) Agora, mostraremos a convergência na parte à direita de (2.28). Como $0<\alpha^{\prime}<\alpha$, temos

$$
d_{\alpha^{\prime}}\left(\frac{\sum_{k=1}^{n} Y_{k}(i)}{n^{\frac{1}{\alpha}}}, Z\right)=d_{\alpha^{\prime}}\left(\frac{\sum_{k=1}^{n} Y_{k}(i)}{n^{\frac{1}{\alpha}}}, \frac{\sum_{k=1}^{n} Z_{k}^{*}}{n^{\frac{1}{\alpha}}}\right) \stackrel{n \rightarrow \infty}{\longrightarrow} 0 .
$$

Por (2.30) e a Proposição 1.3(a), temos para $\alpha^{\prime}<\alpha$,

$$
E\left\{\left|\frac{\sum_{k=1}^{n} Z_{k}^{*}}{n^{\frac{1}{\alpha}}}\right|\right\}=E\left\{|Z|^{\alpha^{\prime}}\right\}<\infty
$$

Segue que

$$
E\left\{\left.\left|\frac{\sum_{k=1}^{n} Y_{k}(i)}{n^{\frac{1}{\alpha}}}\right|\right|^{\alpha^{\prime}}\right\} \stackrel{n \rightarrow \infty}{\longrightarrow} E\left\{|Z|^{\alpha^{\prime}}\right\}
$$

Seja $\left\{l_{n}\right\}$ definido por (2.2). Agora, nós estendemos os resultados acima para a sequência de índices aleatórios $\left\{l_{n}\right\}$. Note que, como $E\left\{|Z|^{\alpha}\right\}=\infty$, nós não podemos esperar $E\left\{\left|\frac{\sum_{k=1}^{l_{n}-1} Y_{k}(i)}{\left(l_{n}-1\right)^{\frac{1}{\alpha}}}\right|^{\alpha}\right\}<\infty$. Assim, o Teorema 1.1 não pode ser usado como no caso Gaussiano.

Teorema 2.6 Assuma que a Condição 2.4 vale para o estado $i$ e seja $Z \stackrel{d}{=} S_{\alpha}\left(\sigma_{i}, 0,0\right)$ para algum 
$\sigma_{i}>0$. Então

$$
d_{\alpha}\left(\frac{\sum_{k=1}^{l_{n}-1} Y_{k}(i)}{\left(l_{n}-1\right)^{\frac{1}{\alpha}}}, Z\right) \stackrel{n \rightarrow \infty}{\longrightarrow} 0 .
$$

Além disso, para $1<\alpha^{\prime}<\alpha$, temos

$$
\frac{\sum_{k=1}^{l_{n}-1} Y_{k}(i)}{\left(l_{n}-1\right)^{\frac{1}{\alpha}}} \stackrel{d}{\longrightarrow} Z \quad e \quad E\left\{\left|\frac{\mid \sum_{k=1}^{l_{n}-1} Y_{k}(i)}{\left(l_{n}-1\right)^{\frac{1}{\alpha}}}\right|^{\alpha^{\prime}}\right\} \stackrel{n \rightarrow \infty}{\longrightarrow} E\left\{|Z|^{\alpha^{\prime}}\right\} .
$$

Demonstração. Como acima, sejam $Z_{1}{ }^{*}, Z_{2}{ }^{*}, \ldots$, cópias independentes de $Z$, tais que

$$
d_{\alpha}{ }^{\alpha}\left(Y_{k}(i), Z_{k}^{*}\right)=E\left\{\left|Y_{k}(i)-Z_{k}{ }^{*}\right|^{\alpha}\right\}, \quad k=1,2, l_{n}-1 .
$$

Para provarmos (2.32), observe que $l_{n}$ é determinado por $\left(\tau_{l_{n}} \leq n, \tau_{l_{n}+1}>n\right)$, tal que $l_{n}$ é $\sigma\left(X_{\tau_{l_{n}}}, X_{\tau_{l_{n}}+1}, \ldots, X_{\tau_{l_{n}+2}-1}\right)$-mensurável. Por outro lado, $Z_{k}{ }^{*}$ depende da cadeia $\left\{X_{n}\right\}_{n \geq 0}$ somente em relação a $Y_{k}(i)$, sendo independente de $Y_{k^{\prime}}(i)$, para $k^{\prime} \neq k$. Dessa forma, segue que a soma parcial $\sum_{k=1}^{l_{n}-1}\left(Y_{k}(i)-Z_{k}{ }^{*}\right)$ é $\sigma\left(Z_{1}{ }^{*}, \ldots, Z_{l_{n}-1}{ }^{*}, X_{\tau_{1}}, \ldots, X_{\tau_{1}+1}, \ldots, X_{\tau_{l_{n}}-1}\right)$-mensurável. Pela Proposição 1.1(b), temos que $l_{n}$ e $\sum_{k=1}^{l_{n}-1}\left(Y_{k}(i)-Z_{k}{ }^{*}\right)$ são independentes. Além disso, $\sum_{k=1}^{l_{n}-1}\left(Y_{k}(i)-Z_{k}{ }^{*}\right)$ é uma soma de variáveis aleatórias i.i.d. com média zero. Assim, $\left\{\sum_{k=1}^{l_{n}-1}\left(Y_{k}(i)-Z_{k}{ }^{*}\right)\right\}_{n \geq 1}$ forma uma martingale. Como $1<\alpha<2$, obtemos, pela desigualdade (1.16),

$$
\begin{aligned}
E\left\{\left|\sum_{k=1}^{l_{n}-1}\left(Y_{k}(i)-Z_{k}{ }^{*}\right)\right|^{\alpha} \mid l_{n}\right\} & \leq 2 E\left\{\sum_{k=1}^{l_{n}-1}\left|Y_{k}(i)-Z_{k}{ }^{*}\right|^{\alpha} \mid l_{n}\right\} \\
& =2\left(l_{n}-1\right) E\left\{\left|Y_{k}(i)-Z_{k}{ }^{*}\right|^{\alpha} \mid l_{n}\right\} \\
& =2\left(l_{n}-1\right) d_{\alpha}{ }^{\alpha}\left(Y_{k}(i), Z_{k}{ }^{*}\right) .
\end{aligned}
$$




\section{Segue que}

$$
\frac{1}{\left(l_{n}-1\right)} E\left\{\left|\sum_{k=1}^{l_{n}-1}\left(Y_{k}(i)-Z_{k}{ }^{*}\right)\right|^{\alpha} \mid l_{n}\right\} \leq 2 d_{\alpha}{ }^{\alpha}\left(Y_{k}(i), Z_{k}{ }^{*}\right)<\infty .
$$

Pelo Lema 1.2, temos a convergência

$$
\frac{1}{\left(l_{n}-1\right)} E\left\{\left|\sum_{k=1}^{l_{n}-1}\left(Y_{k}(i)-Z_{k}^{*}\right)\right|^{\alpha} \mid l_{n}\right\} \stackrel{\text { q.c. }}{\longrightarrow} 0 .
$$

Junto à limitação em (2.34), obtemos, pelo Teorema da Convergência Limitada,

$$
E\left\{\left|\frac{\sum_{k=1}^{l_{n}-1}\left(Y_{k}(i)-Z_{k}^{*}\right)}{\left(l_{n}-1\right)^{\frac{1}{\alpha}}}\right|^{\alpha}\right\} \stackrel{n \rightarrow \infty}{\longrightarrow} 0 .
$$

Agora, considerando a função característica, temos que

$$
\begin{aligned}
E\left\{e^{\left(i t \frac{1}{\left(l_{n}-1\right)^{\frac{1}{\alpha}}} \sum_{k=1}^{l_{n}-1} Z_{k}^{*}\right)} \mid l_{n}=m\right\} & =E\left\{e^{\left(i \frac{1}{(m-1) \frac{1}{\alpha}} \sum_{k=1}^{m-1} Z_{k}^{*}\right)}\right\} \\
& =E\left\{e^{i t Z}\right\} .
\end{aligned}
$$

$\left.\operatorname{Logo}_{0} E\left\{e^{\left(i t \frac{1}{\left(l_{n}-1\right)^{\frac{1}{\alpha}}} \sum_{k=1}^{l_{n}-1} Z_{k}^{*}\right.}\right) \mid l_{n}\right\}=E\left\{e^{i t Z}\right\}$. Portanto,

$$
\begin{aligned}
E\left\{e^{\left(i t \frac{1}{\left(l_{n}-1\right) \frac{1}{\alpha}} \sum_{k=1}^{l_{n}-1} Z_{k}^{*}\right)}\right\} & \left.=E\left\{E\left\{e^{\left(i t \frac{1}{\left(l_{n}-1\right)^{\frac{1}{\alpha}}} \sum_{k=1}^{l_{n}-1} Z_{k}^{*}\right.}\right) \mid l_{n}\right\}\right\} \\
& =E\left\{e^{i t Z}\right\} .
\end{aligned}
$$

Segue que 


$$
d_{\alpha}\left(\frac{\sum_{k=1}^{l_{n}-1} Y_{k}(i)}{\left(l_{n}-1\right)^{\frac{1}{\alpha}}}, Z\right)=d_{\alpha}\left(\frac{\sum_{k=1}^{l_{n}-1} Y_{k}(i)}{\left(l_{n}-1\right)^{\frac{1}{\alpha}}}, \frac{\sum_{k=1}^{l_{n}-1} Z_{k}{ }^{*}}{\left(l_{n}-1\right)^{\frac{1}{\alpha}}}\right),
$$

e, assim, obtemos (2.32). Claramente, a convergência em distribuição é uma consequência imediata. Para $1<\alpha^{\prime}<\alpha$, a convergência dos momentos de ordem $\alpha^{\prime}$ pode ser obtida usando os mesmos argumentos como na demonstração de (2.28).

\section{Sejam}

$$
\begin{gathered}
\widetilde{V}_{\tau_{l_{n}}-1}=\sum_{l=\tau_{1}(i)}^{\tau_{l_{n}}(i)-1}\left(\phi\left(X_{l}\right)-\mu_{\phi}\right), \\
V_{l_{n}-1}=\sum_{k=1}^{l_{n}-1} Y_{k}(i) \text { e } \stackrel{Z}{V} l_{l_{n}-1}=\sum_{k=1}^{l_{n}-1} Z_{k}{ }^{*},
\end{gathered}
$$

onde $Z_{1}{ }^{*}, Z_{2}{ }^{*}, \ldots$, são cópias independentes de $Z$ como em (2.35). Claramente, temos que

$$
\frac{\widetilde{V}_{\tau_{l_{n}}-1}}{\tau_{l_{n}}{ }^{\frac{1}{\alpha}}}=\left(\frac{l_{n}-1}{\tau_{l_{n}}}\right)^{\frac{1}{\alpha}} \frac{V_{l_{n}-1}}{\left(l_{n}-1\right)^{\frac{1}{\alpha}}},
$$

e, se as hipóteses do Teorema 2.6 estão satisfeitas, então

$$
\begin{aligned}
d_{\alpha}^{\alpha}\left(\frac{\widetilde{V}_{\tau_{l_{n}}-1}}{\tau_{l_{n}} \frac{1}{\alpha}},\left(\frac{l_{n}-1}{\tau_{l_{n}}}\right)^{\frac{1}{\alpha}} \frac{\stackrel{Z}{V_{l_{n}-1}}}{\left(l_{n}-1\right)^{\frac{1}{\alpha}}}\right) & =d_{\alpha}^{\alpha}\left(\left(\frac{l_{n}-1}{\tau_{l_{n}}}\right)^{\frac{1}{\alpha}} \frac{V_{l_{n}-1}}{\left(l_{n}-1\right)^{\frac{1}{\alpha}}},\left(\frac{l_{n}-1}{\tau_{l_{n}}}\right)^{\frac{1}{\alpha}} \frac{Z}{V_{l_{n}-1}}\right. \\
& \leq E\left\{\frac{l_{n}-1}{\left.l_{l_{n}}-1\right)^{\frac{1}{\alpha}}}\left|\frac{V_{l_{n}-1}}{\left(l_{n}-1\right)^{\frac{1}{\alpha}}}-\frac{\stackrel{Z}{V} l_{n}-1}{\left(l_{n}-1\right)^{\frac{1}{\alpha}}}\right|^{\alpha}\right\} \\
& \leq E\left\{\left|\frac{V_{l_{n}-1}}{\left(l_{n}-1\right)^{\frac{1}{\alpha}}}-\frac{V_{l_{n}-1}}{\left(l_{n}-1\right)^{\frac{1}{\alpha}}}\right|^{\alpha}\right\} \\
& =d_{\alpha}^{\alpha}\left(\frac{V_{l_{n}-1}}{\left(l_{n}-1\right)^{\frac{1}{\alpha}}}, Z\right) \stackrel{n \rightarrow \infty}{\longrightarrow} 0 .
\end{aligned}
$$

Observe que usamos o fato que $\frac{l_{n}-1}{\tau_{l_{n}}} \leq 1$ e (2.32), para obtermos a última convergência acima. Isso nos permite concluir 


$$
\frac{\widetilde{V}_{\tau_{l_{n}-1}}}{\tau_{l_{n}}^{\frac{1}{\alpha}}}-\frac{Z}{V_{l_{n}-1}}{\frac{p}{\tau_{l_{n}} \frac{1}{\alpha}}}_{\longrightarrow}^{\longrightarrow}
$$

Por outro lado,

$$
\frac{\stackrel{Z}{V}}{\tau_{l_{n}-1}}=\left(\frac{l_{n}-1}{\tau_{l_{n}} \frac{1}{\alpha}}\right)^{\frac{1}{\alpha}} \frac{\stackrel{Z}{V} l_{l_{n}-1}}{\left(l_{n}-1\right)^{\frac{1}{\alpha}}} \stackrel{d}{=}\left(\frac{l_{n}-1}{\tau_{l_{n}}}\right)^{\frac{1}{\alpha}} Z \stackrel{d}{\longrightarrow} \pi_{i^{\frac{1}{\alpha}}} Z .
$$

A última convergência segue pelo fato que $\frac{l_{n}-1}{\tau_{l_{n}}} \stackrel{\text { q.c. }}{\longrightarrow} \pi_{i}$. Agora, usando o fato que, se $\psi_{n} \stackrel{d}{\longrightarrow} \psi \mathrm{e}$ $\eta_{n} \stackrel{p}{\longrightarrow} 0$, então $\psi_{n}+\eta_{n} \stackrel{d}{\longrightarrow} \psi$, nós concluímos que

$$
\frac{\widetilde{V}_{\tau_{l_{n}}-1}}{\tau_{l_{n}} \frac{d}{\alpha}} \stackrel{d}{\longrightarrow} \pi_{i}^{\frac{1}{\alpha}} Z \text { ou } \quad \frac{\widetilde{V}_{\tau_{l_{n}}-1}}{\pi_{i^{\frac{1}{\alpha}} \tau_{l_{n}} \frac{1}{\alpha}}} \stackrel{d}{\longrightarrow} Z .
$$

Como nós não temos $E\left\{|Z|^{\alpha}\right\}<\infty$, não podemos proceder como no caso Gaussiano para obtermos

$$
d_{\alpha}\left(\frac{\widetilde{V}_{\tau_{l_{n}}-1}}{\tau_{l_{n}}^{\frac{1}{\alpha}}}, \pi_{i}^{\frac{1}{\alpha}} Z\right) \stackrel{n \rightarrow \infty}{\longrightarrow} 0 .
$$

Alternativamente, pela Proposição 1.3, temos $E\left\{|Z|^{\alpha^{\prime}}\right\}<\infty$ para $0<\alpha^{\prime}<\alpha$. Se tomarmos $0<\alpha^{\prime}<\alpha$, por (1.12) nós obtemos

$d_{\alpha^{\prime}}\left(\frac{\widetilde{V}_{\tau_{l_{n}}-1}}{\tau_{l_{n}} \frac{1}{\alpha}}, \pi_{i}^{\frac{1}{\alpha}} Z\right) \leq d_{\alpha^{\prime}}\left(\frac{\widetilde{V}_{\tau_{l_{n}}-1}}{\tau_{l_{n}} \frac{1}{\alpha}},\left(\frac{l_{n}-1}{\tau_{l_{n}}}\right)^{\frac{1}{\alpha}} \frac{\stackrel{Z}{V} l_{l_{n}-1}}{\left(l_{n}-1\right)^{\frac{1}{\alpha}}}\right)+d_{\alpha^{\prime}}\left(\left(\frac{l_{n}-1}{\tau_{l_{n}}}\right)^{\frac{1}{\alpha}} \frac{\stackrel{Z}{V} l_{l_{n}-1}}{\left(l_{n}-1\right)^{\frac{1}{\alpha}}}, \pi_{i}^{\frac{1}{\alpha}} Z\right)$.

Pela prova do Teorema 2.6, obtemos

$$
\begin{aligned}
d_{\alpha^{\prime}}\left(\left(\frac{l_{n}-1}{\tau_{l_{n}}}\right)^{\frac{1}{\alpha}} \frac{Z}{V_{l_{n}-1}}\left(l_{n}-1\right)^{\frac{1}{\alpha}}, \pi_{i}^{\frac{1}{\alpha}} Z\right) & =d_{\alpha^{\prime}}\left(\left(\frac{l_{n}-1}{\tau_{l_{n}}}\right)^{\frac{1}{\alpha}} Z, \pi_{i}^{\frac{1}{\alpha}} Z\right) \\
& \leq E\left\{|Z|^{\alpha^{\prime}}\left|\left(\frac{l_{n}-1}{\tau_{l_{n}}}\right)^{\frac{1}{\alpha}}-\pi_{i^{\frac{1}{\alpha}}}\right|^{\alpha^{\prime}}\right\} \stackrel{n \rightarrow \infty}{\longrightarrow} 0 .
\end{aligned}
$$

Por (2.4), temos que $\left|\left(\frac{l_{n}-1}{\tau_{l_{n}}}\right)^{\frac{1}{\alpha}}-\pi_{i}^{\frac{1}{\alpha}}\right| \stackrel{\text { q.c. }}{\longrightarrow} 0$ e como $E\left\{|Z|^{\alpha^{\prime}}\right\}<\infty$, obtemos a última convergência como consequência do Teorema da Convergência Dominada. Atrelado a (2.38), isso nos 
dá

$$
d_{\alpha^{\prime}}\left(\frac{\widetilde{V}_{\tau_{l_{n}}-1}}{\tau_{l_{n}} \frac{1}{\alpha}}, \pi_{i}^{\frac{1}{\alpha}} Z\right) \stackrel{n \rightarrow \infty}{\longrightarrow} 0 .
$$

Agora, o Lema 2.3(c) mostra que se a Condição 2.4 é satisfeita para algum estado $i$, então temos $d_{\alpha^{\prime}}\left(Y_{k}(j), Z\right)<\infty, \forall j \in S$. Assim, (2.39) e (2.40) continuam válidas se o estado $i$ é trocado por qualquer outro estado $j \in S$. Claramente, neste caso, $l_{n}$ e $\tau_{1}(j), \tau_{2}(j), \ldots$, terão que ser definidos como em (2.1), trocando $i$ por $j$.

Teorema 2.7 Assuma que a Condição 2.4 vale para o estado i e seja $Z \stackrel{d}{=} S_{\alpha}\left(\sigma_{i}, 0,0\right)$ para algum $\sigma_{i}>0$. Seja $1<\alpha^{\prime}<\alpha$. Então, para $j \in S$, existe $Z(j) \stackrel{d}{=} S_{\alpha}\left(\sigma_{j}, 0,0\right)$, tal que

$$
\begin{gathered}
d_{\alpha^{\prime}}\left(\frac{\widetilde{V}_{\tau_{l_{n}}(j)-1}}{\left.\pi_{j^{\frac{1}{\alpha}} \tau_{l_{n}} \frac{1}{\alpha}(j)}, Z(j)\right) \stackrel{n \rightarrow \infty}{\longrightarrow} 0,}\right. \\
\frac{\widetilde{V}_{\tau_{l_{n}}(j)-1}}{\pi_{j^{\frac{1}{\alpha}}} \tau_{l_{n}} \frac{1}{\alpha}(j)} \stackrel{d}{\longrightarrow} Z(j) \quad e \quad E\left\{\left|\frac{\widetilde{V}_{\tau_{l_{n}}(j)-1}}{\pi_{j}^{\frac{1}{\alpha}} \tau_{l_{n}} \frac{1}{\alpha}(j)}\right|^{\alpha^{\prime}}\right\} \stackrel{n \rightarrow \infty}{\longrightarrow} E\left\{|Z(j)|^{\alpha^{\prime}}\right\} .
\end{gathered}
$$

Nós podemos reescrever (2.41) e (2.42) como

$$
\begin{gathered}
d_{\alpha^{\prime}}\left(\frac{\widetilde{V}_{\tau_{l_{n}}(j)-1}}{\tau_{l_{n}}^{\frac{1}{\alpha}}(j)}, \pi_{j}^{\frac{1}{\alpha}} Z(j)\right) \stackrel{n \rightarrow \infty}{\longrightarrow} 0, \\
\frac{\widetilde{V}_{\tau_{l_{n}}(j)-1} \stackrel{d}{\tau_{l_{n}} \frac{1}{\alpha}(j)} \pi_{j}^{\frac{1}{\alpha}} Z(j)}{e} \quad E\left\{\left|\frac{\widetilde{V}_{\tau_{l_{n}}(j)-1}}{\tau_{l_{n}}^{\frac{1}{\alpha}}(j)}\right|^{\alpha^{\prime}}\right\} \stackrel{n \rightarrow \infty}{\longrightarrow} E\left\{\pi_{j}^{\frac{\alpha^{\prime}}{\alpha}}|Z(j)|^{\alpha^{\prime}}\right\} .
\end{gathered}
$$

Nós mostraremos adiante que

$$
\pi_{j}^{\frac{1}{\alpha}} Z(j) \stackrel{d}{=} \pi_{j^{\prime}} \frac{1}{\alpha} Z\left(j^{\prime}\right) \stackrel{d}{=} S_{\alpha}\left(\pi_{j}^{\frac{1}{\alpha}} \sigma_{j}, 0,0\right)=S_{\alpha}(\sigma, 0,0) .
$$

Ou seja, temos que $\pi_{j}^{\frac{1}{\alpha}} \sigma_{j}=\sigma, \forall j \in S$. Assim, de maneira a estender esses resultados para a soma parcial $S_{n}-n \mu_{\phi}=\sum_{l=0}^{n}\left(\phi\left(X_{l}\right)-\mu_{\phi}\right)$, não há perda de generalidade ao fixarmos o estado $i$ que satisfaz a Condição 2.4.

Com $\widetilde{V}_{\tau_{l_{n}}-1}$ dado por (2.37), seja

$$
S_{n}-n \mu_{\phi}=A_{n}(i)+\widetilde{V}_{\tau_{l_{n}-1}}+B_{n}(i),
$$


onde $A_{n}(i)=\sum_{l=0}^{\tau_{1}-1}\left(\phi\left(X_{l}\right)-\mu_{\phi}\right)$ e $B_{n}(i)=\sum_{l=\tau_{l_{n}}}^{n}\left(\phi\left(X_{l}\right)-\mu_{\phi}\right)$. Para controlar os termos $A_{n}(i)$ e $B_{n}(i)$, nossas provas necessitarão que $E_{\pi}\left\{\left|\phi\left(X_{k}\right)-\mu_{\phi}\right|^{\alpha^{\prime}}\right\}<\infty, 0 \leq k \leq 1$, para $\alpha^{\prime}<\alpha$. Uma condição suficiente para isso é $d_{\alpha}\left(\phi\left(X_{k}\right), Z_{0}\right)<\infty$, para alguma variável aleatória $\alpha$-estável $Z_{0}$. Como no caso Gaussiano, nós conjeturamos que a condição $d_{\alpha}\left(Y_{k}(i), Z\right)<\infty$ é suficiente para mostrar que $d_{\alpha}\left(\phi\left(X_{k}\right), Z_{0}\right)<\infty$, mas nós não estamos hábeis a mostrar isso.

Condição 2.5 Seja X uma cadeia de Markov aperiódica e recorrente positiva. Assuma que para algum $1<\alpha<2$ existe uma variável aleatória $\alpha$-estável $Z(i) \stackrel{d}{=} S_{\alpha}\left(\sigma_{i}, 0,0\right)$ tal que $d_{\alpha}\left(Y_{k}(i), Z(i)\right)<$ $\infty$, para algum $i \in S$ e algum $k \geq 1$. Além disso, assuma que $E_{\pi}\left\{\left|\phi\left(X_{k}\right)-\mu_{\phi}\right|^{\alpha^{\prime}}\right\}<\infty, 0 \leq k \leq 1$, para $1<\alpha^{\prime}<\alpha$.

Teorema 2.8 Assuma que a Condição 2.5 vale para o estado $i$ e seja $Z \stackrel{d}{=} S_{\alpha}(\sigma, 0,0)$, com $\sigma=\pi_{i}^{\frac{1}{\alpha}} \sigma_{i}$. Então para $1<\alpha^{\prime}<\alpha$, temos

$$
\begin{gathered}
d_{\alpha^{\prime}}\left(\frac{S_{n}-n \mu_{\phi}}{\left.n^{\frac{1}{\alpha}}, Z\right) \stackrel{n \rightarrow \infty}{\longrightarrow} 0,}\right. \\
\frac{S_{n}-n \mu_{\phi}}{n^{\frac{1}{\alpha}}} \stackrel{d}{\longrightarrow} Z \quad e \quad E\left\{\left|\frac{S_{n}-n \mu_{\phi}}{n^{\frac{1}{\alpha}}}\right|^{\alpha^{\prime}}\right\} \stackrel{n \rightarrow \infty}{\longrightarrow} E\left\{|Z|^{\alpha^{\prime}}\right\} .
\end{gathered}
$$

Demonstração. (i) Iniciamente, mostramos que

$$
E\left\{\left|\frac{S_{n}-n \mu_{\phi}}{n^{\frac{1}{\alpha}}}\right|^{\alpha^{\prime}}\right\}<\infty .
$$

Por (2.43) e pela desigualdade de Minkowsky, obtemos

$$
\left\|\frac{S_{n}-n \mu_{\phi}}{n^{\frac{1}{\alpha}}}\right\|_{\alpha^{\prime}} \leq\left\|\frac{A_{n}(i)}{n^{\frac{1}{\alpha}}}\right\|_{\alpha^{\prime}}+\left\|\frac{\widetilde{V}_{\tau_{n}-1}}{n^{\frac{1}{\alpha}}}\right\|_{\alpha^{\prime}}+\left\|\frac{B_{n}(i)}{n^{\frac{1}{\alpha}}} \cdot\right\|_{\alpha^{\prime}}
$$

Como $\alpha^{\prime}>1$, pelo Lema 2.2, temos que

$$
\begin{aligned}
E\left\{\left|A_{n}(i)\right|^{\alpha^{\prime}}\right\} & \leq E\left\{\tau_{1}^{\alpha^{\prime}-1} \sum_{l=0}^{\tau_{1}-1}\left|\phi\left(X_{l}\right)-\mu_{\phi}\right|^{\alpha^{\prime}}\right\} \\
& \leq E_{i}\left\{\tau_{1}^{\alpha^{\prime}-1} \sum_{l=0}^{\tau_{1}-1}\left|\phi\left(X_{l}\right)-\mu_{\phi}\right|^{\alpha^{\prime}}\right\}
\end{aligned}
$$

e 


$$
E_{i}\left\{\frac{\tau_{1}^{\alpha^{\prime}-1}}{n^{\frac{\alpha^{\prime}}{\alpha}}} \sum_{l=0}^{\tau_{1}-1}\left|\phi\left(X_{l}\right)-\mu_{\phi}\right|^{\alpha^{\prime}}\right\} \leq \frac{1}{n^{\delta}} E_{i}\left\{\sum_{l=0}^{\tau_{1}-1}\left|\phi\left(X_{l}\right)-\mu_{\phi}\right|^{\alpha^{\prime}}\right\}
$$

Note que $\delta=\frac{\alpha^{\prime}}{\alpha}-\left(\alpha^{\prime}-1\right)>0$ e $\frac{1}{n^{\delta}}\left(\frac{\tau_{1}}{n}\right)^{\alpha^{\prime}-1} \leq \frac{1}{n^{\delta}}$. Como $E_{\pi}\left\{\left|\phi\left(X_{0}\right)-\mu_{\phi}\right|^{\alpha^{\prime}}\right\}<\infty$, nós temos, pelo Lema 2.1(c),

$$
E_{i}\left\{\sum_{l=0}^{\tau_{1}-1}\left|\phi\left(X_{l}\right)-\mu_{\phi}\right|^{\alpha^{\prime}}\right\}=\frac{1}{\pi_{i}} E_{\pi}\left\{\left|\phi\left(X_{0}\right)-\mu_{\phi}\right|^{\alpha^{\prime}}\right\}<\infty .
$$

Segue que

$$
E\left\{\left|\frac{A_{n}(i)}{n^{\frac{1}{\alpha}}}\right|^{\alpha^{\prime}}\right\} \stackrel{n \rightarrow \infty}{\longrightarrow} 0 .
$$

Analogamente,

$$
\begin{aligned}
E\left\{\left|\frac{B_{n}(i)}{n^{\frac{1}{\alpha}}}\right|^{\alpha^{\prime}}\right\} & \leq E\left\{\frac{1}{n^{\frac{\alpha^{\prime}}{\alpha}}}\left(\tau_{l_{n}+1}-\tau_{l_{n}}\right)^{\alpha^{\prime}-1} \sum_{l=\tau_{l_{n}}}^{n}\left|\phi\left(X_{l}\right)-\mu_{\phi}\right|^{\alpha^{\prime}}\right\} \\
& \leq \frac{1}{n^{\delta}} E\left\{\sum_{l=\tau_{l_{n}}}^{\tau_{l_{n}+1}-1}\left|\phi\left(X_{l}\right)-\mu_{\phi}\right|^{\alpha^{\prime}}\right\} \\
& =\frac{1}{n^{\delta}} \frac{1}{\pi_{i}} E_{\pi}\left\{\left|\phi\left(X_{0}\right)-\mu_{\phi}\right|^{\alpha^{\prime}}\right\} \stackrel{n \rightarrow \infty}{\longrightarrow} 0 .
\end{aligned}
$$

Além disso, para o termo $\left\|\frac{\widetilde{V}_{\tau_{l_{n}}-1}}{n^{\frac{1}{\alpha}}}\right\|_{\alpha^{\prime}}$, como $\tau_{l_{n}} \leq n$, nós temos, por (2.42),

$$
\begin{aligned}
E\left\{\left|\frac{\widetilde{V}_{\tau_{l_{n}}-1}}{n^{\frac{1}{\alpha}}}\right|^{\alpha^{\prime}}\right\} & =E\left\{\left(\frac{\tau_{l_{n}}}{n}\right)^{\frac{\alpha^{\prime}}{\alpha}}\left|\frac{\widetilde{V}_{\tau_{l_{n}}-1}}{\tau_{l_{n}} \frac{1}{\alpha}}\right|^{\alpha^{\prime}}\right\} \\
& \leq E\left\{\left|\frac{\widetilde{V}_{\tau_{l_{n}}-1}}{\tau_{l_{n}}{ }^{\frac{1}{\alpha}}}\right|^{\alpha^{\prime}}\right\} \stackrel{n \rightarrow \infty}{\longrightarrow} E\left\{\pi_{i}^{\frac{\alpha^{\prime}}{\alpha}}|Z(i)|^{\alpha^{\prime}}\right\}<\infty,
\end{aligned}
$$

e isso completa a prova de (2.46).

(ii) Agora, nós demonstramos (2.44). Sejam $Z_{1}{ }^{*}, Z_{2}{ }^{*}, \ldots$, cópias independentes de $Z(i) \stackrel{d}{=} S_{\sigma}\left(\sigma_{i}, 0,0\right)$.

Então, como mostrado no Teorema 2.6, temos que $\frac{\sum_{k=1}^{l_{n}-1} Z_{k}{ }^{*}}{\left(l_{n}-1\right)^{\frac{1}{\alpha}}} \stackrel{d}{=} Z(i)$. 
Agora, para $V_{l_{n}-1}=\sum_{k=1}^{l_{n}-1} Y_{k}(i)$, temos

$$
\frac{\widetilde{V}_{\tau_{l_{n}}-1}}{n^{\frac{1}{\alpha}}}=\left(\frac{l_{n}-1}{n}\right)^{\frac{1}{\alpha}} \frac{V_{l_{n}-1}}{\left(l_{n}-1\right)^{\frac{1}{\alpha}}} .
$$

Seja $\stackrel{Z}{V} l_{l_{n}-1}=\sum_{k=1}^{l_{n}-1} Z_{k}{ }^{*}$. Assim, temos que

$$
\begin{aligned}
d_{\alpha^{\prime}}{ }^{\alpha^{\prime}}\left(\left(\frac{l_{n}-1}{n}\right)^{\frac{1}{\alpha}} \frac{V_{l_{n}-1}}{\left(l_{n}-1\right)^{\frac{1}{\alpha}}},\left(\frac{l_{n}-1}{n}\right)^{\frac{1}{\alpha}} Z(i)\right) & =d_{\alpha^{\prime}}{ }^{\alpha^{\prime}}\left(\left(\frac{l_{n}-1}{n}\right)^{\frac{1}{\alpha}} \frac{V_{l_{n}-1}}{\left(l_{n}-1\right)^{\frac{1}{\alpha}}},\left(\frac{l_{n}-1}{n}\right)^{\frac{1}{\alpha}} \frac{Z}{V_{l_{n}-1}}\right. \\
& \leq E\left\{\left(\frac{l_{n}-1}{n}\right)^{\frac{\alpha^{\prime}}{\alpha}} \mid \frac{V_{l_{n}-1}}{\left(l_{n}-1\right)^{\frac{1}{\alpha}}}-\frac{V_{l_{n}-1}}{\left(l_{n}-1\right)^{\frac{1}{\alpha}}}\right\} \\
& \leq E\left\{\left|\frac{V_{l_{n}-1}^{\alpha^{\prime}}}{\left(l_{n}-1\right)^{\frac{1}{\alpha}}}-\frac{V_{l_{n}-1}}{\left(l_{n}-1\right)^{\frac{1}{\alpha}}}\right|^{\alpha^{\prime}} \underset{n \rightarrow \infty}{\longrightarrow} 0,\right.
\end{aligned}
$$

onde a última convergência segue de (2.35).

Como $Z(i) \stackrel{d}{=} \frac{1}{\pi_{i}^{\frac{1}{\alpha}}} Z$ e $E\left\{|Z|^{\alpha^{\prime}}\right\}<\infty$, pelo Teorema da Convergência Dominada, temos que

$$
\begin{aligned}
d_{\alpha^{\prime}}{ }^{\alpha^{\prime}}\left(\left(\frac{l_{n}-1}{n}\right)^{\frac{1}{\alpha}} Z(i), Z\right) & \leq E\left\{\left|\left(\frac{l_{n}-1}{n}\right)^{\frac{1}{\alpha}} Z(i)-Z\right|^{\alpha^{\prime}}\right\} \\
& =E\left\{\left(\left(\frac{l_{n}-1}{n}\right)^{\frac{1}{\alpha}} \frac{1}{\pi_{i}^{\frac{1}{\alpha}}}-1\right)^{\alpha^{\prime}}|Z|^{\alpha^{\prime}}\right\} \stackrel{n \rightarrow \infty}{\longrightarrow} 0
\end{aligned}
$$

onde para última convergência nós usamos o fato de que $\left(\frac{l_{n}-1}{n}\right)^{\frac{1}{\alpha}} \frac{1}{\pi_{i}^{\frac{1}{\alpha}}} \stackrel{q \cdot c .}{\longrightarrow} 1$ (conforme o Lema 2.1(a)).

(iii) Pelo Teorema 1.1, nós concluímos a prova.

Observação 2.2 Claramente, $\pi_{i}{ }^{\frac{1}{\alpha}} \sigma_{i}=\sigma$ é independente de $i$. Caso contrário, escolhendo-se $j \neq i$, teríamos que

$$
\frac{S_{n}-n \mu_{\phi}}{n^{\frac{1}{\alpha}}} \stackrel{d}{\longrightarrow} Z \stackrel{d}{=} S_{\alpha}\left(\pi_{i}^{\frac{1}{\alpha}} \sigma_{i}, 0,0\right)
$$


$e$

$$
\frac{S_{n}-n \mu_{\phi}}{n^{\frac{1}{\alpha}}} \stackrel{d}{\longrightarrow} Z^{\prime} \stackrel{d}{=} S_{\alpha}\left(\pi_{j^{\frac{1}{\alpha}}} \sigma_{j}, 0,0\right) .
$$

Mas isso é uma contradição. 


\section{Capítulo 3}

\section{Convergência de Processos Empíricos Associados a Cadeias de Markov com Espaço de Estados Geral}

\subsection{Introdução}

Neste capítulo, provamos a convergência fraca dos processos empírico e quantil empírico associados a uma cadeia de Markov com espaço de estados geral e ergódica. Existem diversas referências na literatura provando esses resultados para o caso onde as variáveis aleatórias associadas são i.i.d. . Em nosso trabalho, buscamos estender o caso i.i.d, através de técnicas alternativas como a distância Mallows, para o caso empírico, e o Lema de Vervaat, para o caso quantil empírico.

Na Seção 3.2 provamos que o processo empírico $\beta_{n}(x)$ converge fracamente para a Ponte Browniana $B(F(x)$ ), tanto para $x$ fixado como no sentido de processos. Para $x$ fixado, ao observarmos que, embora $\left\{X_{n}\right\}_{n \geq 0}$ seja uma cadeia de Markov com espaço de estados geral, o funcional $\mathbb{I}_{\left[X_{l} \leq x\right]}$ é uma cadeia de Markov discreta, o resultado pode ser obtido como uma simples adaptação do Teorema Ergódico para cadeias de Markov discretas, conforme consta em textos como em Chung (1960). Também existe, ainda, uma outra forma de obtermos esse resultado inicial, conforme está construída em Meyn e Tweedie (2009), mas usando conceitos muito complexos relativos a cadeias de Markov com espaço de estados geral, que fogem ao objetivo desta tese. Apesar disso, obtivemos esse resultado no Lema 3.2 como uma simples consequência da convergência na distância Mallows demonstrada no Lema 3.1.

De fato, o uso da distância Mallows é imprescindível para obtermos, no Teorema 3.1, a convergência entre as respectivas distribuições de dimensão finita de $\beta_{n}(x)$ e $B(F(x))$ que, atrelada à rigidez de $\left\{\beta_{n}\right\}$ provada no Teorema 3.2, demonstram a convergência fraca, no sentido de processos, do processo empírico $\beta_{n}(x)$ para a Ponte Browniana $B(F(x))$.

Uma vez obtida a convergência fraca do processo empírico, o passo lógico seguinte é provar a convergência fraca para o caso quantil empírico. Para o caso i.i.d., muito sobre a teoria e resultados 
relacionados ao processo quantil empírico pode ser estudado em Shorack e Wellner (1986) e Csörgo e Révész (1981), dentre outras referências. Assim, também estendendo o caso i.i.d, na Seção 3.3 provamos, sob condições adequadas e com as variáveis aleatórias associadas constituindo uma cadeia de Markov com espaço de estados geral e ergódica, a convergência fraca do processo quantil empírico $q_{n}(t)$.

Há uma certa dificuldade na tentativa de tratamento do processo quantil empírico com a mesma metodologia adotada para o caso empírico. Assim, algum método alternativo é necessário para eliminarmos essa dificuldade. Nossa estratégia, dessa forma, é provar o caso quantil empírico usando o caso empírico já demonstrado na Seção 3.2. Nossa primeira tentativa foi usar o chamado Método Delta, muito utilizado em referências estatísticas. Porém, a aplicação desse artifício mostrou-se inconsistente e, assim, procuramos na literatura um outro método que fosse coerente com a teoria probabilística referente ao nosso objetivo. Dessa maneira, o método encontrado, e que nos é de fundamental importância, consiste basicamente em usar o Lema de Vervaat atrelado ao Teorema de Skorohod, com a mesma abordagem adotada em Vervaat (1971), Haan e Ferreira (2006) e Resnick (2007).

Assim, na Subseção 3.3.1, como uma forma de nos habituarmos e entendermos melhor a demonstração do caso geral da subseção seguinte, provamos a convergência em distribuição do processo quantil empírico $q_{n}(t)$, com $t$ fixado. Dessa forma, no Teorema 3.4 mostramos o caso quantil uniforme e, no Teorema 3.5, provamos o caso não-uniforme.

Finalmente, na Subseção 3.3.2, através da mesma metodologia utilizada na Subseção 3.3.1, generalizamos os resultados obtidos ao provarmos a convergência fraca, no sentido de processos, do processo quantil empírico $q_{n}(t)$, com $t \in(0,1)$.

\subsection{Convergênca Fraca do Processo Empírico}

Nesta seção, provaremos a convergência fraca do processo empírico, associado a uma cadeia de Markov $\left\{X_{n}\right\}_{n \geq 0}$ ergódica e com espaço de estados geral, para a Ponte Browniana $B(F(x))$, ao obtermos a convergência das distribuições finitas e a rigidez de $\left\{\beta_{n}\right\}$. Para que a definição do processo empírico e nossos resultados façam sentido, como a cadeia é ergódica, durante todo esse capítulo vamos tomar $F(x)=E_{\pi}\left(\phi\left(X_{k}\right)\right)$, para $0 \leq k \leq n$, onde $\pi$ é a distribuição limite da cadeia.

Inicialmente, note que $\mathbb{I}_{\left[X_{l} \leq x\right]}$ torna-se uma cadeia de Markov discreta com espaço de estados $\{0,1\}$. Além disso, não é difícil verificar que essa cadeia é ergódica. Assim, podemos então aplicar os resultados do caso Gaussiano do Capítulo 2 ao processo empírico. De fato, podemos reescrever $\beta_{n}(x)$ da seguinte maneira:

$$
\beta_{n}(x)=\frac{\sum_{l=1}^{n}\left(\mathbb{I}_{\left[X_{l} \leq x\right]}-F(x)\right)}{\sqrt{n}} .
$$


Dessa forma, identificando $\phi\left(X_{l}\right)=\mathbb{I}_{\left[X_{l} \leq x\right]}$ e $\mu_{\phi}=E_{\pi}\left(\phi\left(X_{k}\right)\right)=F(x), 0 \leq k \leq n$, como consequência do Corolário 2.1, obtemos o seguinte lema.

Lema 3.1 Seja $\left\{X_{n}\right\}_{n \geq 0}$ uma cadeia de Markov com espaço de estados geral e ergódica. Então, se $0<F(x)<1$ para $x \in \mathbb{R}$ fixado, temos

$$
d_{2}\left(\beta_{n}(x), B(F(x))\right) \stackrel{n \rightarrow \infty}{\longrightarrow} 0
$$

onde $B(F(x)) \stackrel{D}{=} \mathcal{N}(0, F(x)(1-F(x)))$.

Demonstraçãa. Como $\mathbb{I}_{\left[X_{l} \leq x\right]}=\{0,1\}$ e $0<F(x)<1$, as hipóteses do Corolário 2.1 estão satisfeitas. Logo, com $\sigma_{\phi}=\sqrt{F(x)(1-F(x))}$ sendo a variância assintótica, temos que

$$
d_{2}\left(\frac{\beta_{n}(x)}{\sqrt{F(x)(1-F(x))}}, Z_{0}\right) \stackrel{n \rightarrow \infty}{\longrightarrow} 0,
$$

onde $Z_{0} \stackrel{d}{=} \mathcal{N}(0,1)$. Mas isso é equivalente ao que queríamos provar.

Com essa mesma argumentação, pelo Corolário 2.1, obtemos o seguinte lema:

Lema 3.2 Seja $\left\{X_{n}\right\}_{n \geq 0}$ uma cadeia de Markov com espaço de estados geral e ergódica. Então, se $0<F(x)<1$ para $x \in \mathbb{R}$ fixado, temos

$$
\left.\beta_{n}(x) \stackrel{D}{\longrightarrow} B(F(x))\right),
$$

onde $B(F(x)) \stackrel{d}{=} \mathcal{N}(0, F(x)(1-F(x)))$.

\subsubsection{Convergência no Sentido das Distribuições Finitas}

A seguir, demonstramos a convergência do processo empírico $\beta_{n}(x)$ para a Ponte Browniana $B(F(x))$, no sentido das distribuições de dimensão finita. Para simplificar nossos cálculos denotaremos $B\left(F\left(x_{i}\right)\right)=B_{x_{i}}$, para $x_{i}$ fixado.

Teorema 3.1 Para fixados $x_{1}, x_{2}, \ldots, x_{k} \in \mathbb{R}, \forall k \in \mathbb{N}$, temos

$$
\left(\beta_{n}\left(x_{1}\right), \beta_{n}\left(x_{2}\right) \ldots, \beta_{n}\left(x_{k}\right)\right) \stackrel{d}{\longrightarrow}\left(B_{x_{1}}, B_{x_{2}}, \ldots, B_{x_{k}}\right) .
$$

Demonstração. O caso $k=1$ já está feito no Lema 3.2. Provemos o caso $k=2$. Sejam $a, b \in \mathbb{R}$ e $F_{n, 2}$ e $G_{2}$ as funções de distribuição das variáveis aleatórias

$$
a \beta_{n}\left(x_{1}\right)+b \beta_{n}\left(x_{2}\right) \text { e } a B_{x_{1}}+b B_{x_{2}},
$$


respectivamente.

Pela definição de distância Mallows e a desigualdade clássica

$$
|x+y|^{p} \leq 2^{p-1}\left(|x|^{p}+|y|^{p}\right),
$$

válida para quaisquer números reais $x$ e $y$ sempre que $p \geq 1$, obtemos

$$
\begin{aligned}
d_{2}^{2}\left(F_{n, 2}, G_{2}\right) & \leq E\left|a \beta_{n}\left(x_{1}\right)+b \beta_{n}\left(x_{2}\right)-\left(a B_{x_{1}}+b B_{x_{2}}\right)\right|^{2} \\
& =E\left|a\left(\beta_{n}\left(x_{1}\right)-B_{x_{1}}\right)+b\left(\beta_{n}\left(x_{2}\right)-B_{x_{2}}\right)\right|^{2} \\
& \left.\leq\left. 2\left\{|a|^{2} E \mid \beta_{n}\left(x_{1}\right)-B_{x_{1}}\right)\right|^{2}+|b|^{2} E\left|\beta_{n}\left(x_{2}\right)-B_{x_{2}}\right|^{2}\right\} \\
& =2\left\{|a|^{2} d_{2}^{2}\left(\beta_{n}\left(x_{1}\right), B_{x_{1}}\right)+|b|^{2} d_{2}^{2}\left(\beta_{n}\left(x_{2}\right), B_{x_{2}}\right)\right\},
\end{aligned}
$$

onde a última igualdade advém do Teorema 1.2, $\operatorname{com}\left(\beta_{n}\left(x_{i}\right), B_{x_{i}}\right) \stackrel{d}{=} F_{\beta_{n}\left(x_{i}\right)} \wedge F_{B_{x_{i}}}, i=1,2$. Pelo Lema 3.1 aplicado para $x_{1}$ e $x_{2}$, obtemos que

$$
d_{2}^{2}\left(\beta_{n}\left(x_{i}\right), B_{x_{i}}\right) \stackrel{n \rightarrow \infty}{\longrightarrow} 0, \quad i=1,2
$$

e, assim,

$$
d_{2}\left(F_{n, 2}, G_{2}\right) \stackrel{n \rightarrow \infty}{\longrightarrow} 0
$$

Logo, para quaisquer $a, b \in \mathbb{R}$,

$$
a \beta_{n}\left(x_{1}\right)+b \beta_{n}\left(x_{2}\right) \stackrel{d_{2}}{\longrightarrow} a B_{x_{1}}+b B_{x_{2}},
$$

isto é,

$$
a \beta_{n}\left(x_{1}\right)+b \beta_{n}\left(x_{2}\right) \stackrel{d}{\longrightarrow} a B_{x_{1}}+b B_{x_{2}},
$$

pois as hipóteses do Teorema 1.1 estão naturalmente satisfeitas.

Portanto, pelo Teorema de Cràmer-Wold, concluímos que

$$
\left(\beta_{n}\left(x_{1}\right), \beta_{n}\left(x_{2}\right)\right) \stackrel{d}{\longrightarrow}\left(B_{x_{1}}, B_{x_{2}}\right) .
$$

Para o caso geral, podemos utilizar o princípio da indução finita para obtermos, para $p \geq 1$, a desigualdade

$$
\left|\sum_{j=1}^{k} a_{j}\right|^{p} \leq 2^{(k-1)(p-1)}\left|a_{1}\right|^{p}+\sum_{j=2}^{k} 2^{(k-j+1)(p-1)}\left|a_{j}\right|^{p},
$$

onde $\left\{a_{j}, j=1, \ldots, k\right\} \subset \mathbb{R}$, e adaptar a demonstração com os mesmos argumentos do caso $k=2$. Assim, sejam $a_{1}, a_{2}, \ldots, a_{k} \in \mathbb{R}$ e $F_{n, k}$ e $G_{k}$ as funções de distribuição das variáveis aleatórias

$$
\sum_{j=1}^{k} a_{j} \beta_{n}\left(x_{j}\right) \text { e } \sum_{j=1}^{k} a_{j} B_{x_{j}}
$$


respectivamente.

Pela definição de distância Mallows e a desigualdade (3.1), temos que

$$
\begin{aligned}
d_{2}^{2}\left(F_{n, k}, G_{k}\right) & \leq E\left|\sum_{j=1}^{k} a_{j} \beta_{n}\left(x_{j}\right)-\sum_{j=1}^{k} a_{j} B_{x_{j}}\right|^{2} \\
& =E\left|\sum_{j=1}^{k} a_{j}\left(\beta_{n}\left(x_{j}\right)-B_{x_{j}}\right)\right|^{2} \\
& \leq 2^{(k-1)}\left|a_{1}\right|^{2} E\left|\beta_{n}\left(x_{1}\right)-B_{x_{1}}\right|^{2}+\sum_{j=2}^{k} 2^{(k-j+1)}\left|a_{j}\right|^{2} E\left|\beta_{n}\left(x_{j}\right)-B_{x_{j}}\right|^{2} \\
& =2^{(k-1)}\left|a_{1}\right|^{2} d_{2}^{2}\left(\beta_{n}\left(x_{1}\right), B_{x_{1}}\right)+\sum_{j=2}^{k} 2^{(k-j+1)}\left|a_{j}\right|^{2} d_{2}^{2}\left(\beta_{n}\left(x_{j}\right), B_{x_{j}}\right),
\end{aligned}
$$

onde a última igualdade advém do Teorema $1.2, \operatorname{com}\left(\beta_{n}\left(x_{i}\right), B_{x_{i}}\right) \stackrel{d}{=} F_{\beta_{n}\left(x_{i}\right)} \wedge F_{B_{x_{i}}}, i=1,2, \ldots, k$. Pelo Lema 3.1 aplicado para $x_{1}, x_{2}, \ldots, x_{k}$, obtemos que

$$
d_{2}^{2}\left(\beta_{n}\left(x_{i}\right), B_{x_{i}}\right) \stackrel{n \rightarrow \infty}{\longrightarrow} 0, \quad i=1,2, \ldots, k
$$

e, assim,

$$
d_{2}\left(F_{n, k}, G_{k}\right) \stackrel{n \rightarrow \infty}{\longrightarrow} 0 .
$$

Logo, para quaisquer $a_{1}, a_{2}, \ldots, a_{k} \in \mathbb{R}$,

$$
\sum_{j=1}^{k} a_{j} \beta_{n}\left(x_{j}\right) \stackrel{d_{2}}{\longrightarrow} \sum_{j=1}^{k} a_{j} B_{x_{j}},
$$

ou seja, novamente pelo Teorema 1.1,

$$
\sum_{j=1}^{k} a_{j} \beta_{n}\left(x_{j}\right) \stackrel{d}{\longrightarrow} \sum_{j=1}^{k} a_{j} B_{x_{j}} .
$$

Portanto, novamente pelo Teorema de Cràmer-Wold, segue que

$$
\left(\beta_{n}\left(x_{1}\right), \beta_{n}\left(x_{2}\right), \ldots, \beta_{n}\left(x_{k}\right)\right) \stackrel{d}{\longrightarrow}\left(B_{x_{1}}, B_{x_{2}}, \ldots, B_{x_{k}}\right) .
$$




\subsubsection{A Rigidez de $\left\{\beta_{n}\right\}$}

Na subseção anterior, provamos que as distribuições de dimensão finita do processo empírico convergem para as respectivas distribuições finitas da Ponte Browniana. A seguir, provaremos que $\left\{\beta_{n}\right\}_{n \geq 1}$ é rígida e, assim, concluiremos que $\beta_{n}(x)$ converge fracamente para a Ponte Browniana $B(F(x))$.

Teorema 3.2 Seja $\beta_{n}(x)$ o processo empírico associado a uma cadeia de Markov com espaço de estados geral e ergódica, então $\left\{\beta_{n}\right\}_{n \geq 1}$ é rígida.

Demonstração. Vamos mostrar que $\left\{\beta_{n}\right\}_{n \geq 1}$ satisfaz o Teorema 1.9. Inicialmente, dado $\eta>0$, se tomarmos $a \geq \frac{E\left|\beta_{n}(0)\right|}{\eta}$, obtemos

$$
P\left\{\left|\beta_{n}(0)\right|>a\right\} \leq \frac{E\left|\beta_{n}(0)\right|}{a} \leq \eta .
$$

Assim, agora é suficiente mostrar que, para cada $\eta$ e $\epsilon$ positivos, existem um $\delta$, com $0<\delta<1$, e um inteiro $n_{0}$ tais que, se $n \geq n_{0}$, então

$$
P\left\{\sup _{t \leq s \leq t+\delta}\left|\beta_{n}(s)-\beta_{n}(t)\right| \geq \epsilon\right\} \leq \delta \eta
$$

vale para todo $t$.

Por conveniência de notação, podemos tomar $t=0$ em (3.2), e então é suficiente (pois podemos considerar $2 \epsilon$ ao invés de $\epsilon$ no teorema, e fazermos as adaptações) provarmos que

$$
P\left\{\sup _{0 \leq s \leq \delta}\left|\beta_{n}(s)-\beta_{n}(0)\right|>\epsilon\right\}<\delta \eta .
$$

A inequação acima é suficiente, pois como a cadeia de Markov é ergódica, podemos tomar a distribuição inicial como sendo a estacionária e, assim, as distribuições dos incrementos serão estacionárias.

Além disso, temos que

$$
P\left\{\sup _{0 \leq s \leq \delta}\left|\beta_{n}(s)-\beta_{n}(0)\right|>\epsilon\right\} \leq P\left\{\sup _{0 \leq s \leq \delta}\left|\beta_{n}(s)\right|>\frac{\epsilon}{2}\right\}+P\left\{\sup _{0 \leq s \leq \delta}\left|\beta_{n}(0)\right|>\frac{\epsilon}{2}\right\}
$$

Vamos primeiro provar que $P\left\{\sup _{0 \leq s \leq \delta}\left|\beta_{n}(s)\right|>\frac{\epsilon}{2}\right\}<\frac{\delta \eta}{2}$. Pela definição de supremo, se temos $\sup _{0 \leq s \leq \delta}\left|\beta_{n}(s)\right|>\frac{\epsilon}{2}$, então obrigatoriamente $\left|\beta_{n}(c)\right|>\frac{\epsilon}{2}$, para algum $c$ tal que $0 \leq c \leq \delta$. Assim, temos que $\left[\sup _{0 \leq s \leq \delta}\left|\beta_{n}(s)\right|>\frac{\epsilon}{2}\right] \subset\left[\left|\beta_{n}(c)\right|>\frac{\epsilon}{2}\right]$. Logo, 


$$
P\left\{\sup _{0 \leq s \leq \delta}\left|\beta_{n}(s)\right|>\frac{\epsilon}{2}\right\} \leq P\left\{\left|\beta_{n}(c)\right|>\frac{\epsilon}{2}\right\}
$$

Pelo Lema 3.2, e tomando $0<\delta<1$ tal que $F(c) \leq \delta$ e $\frac{\delta^{2} \cdot 3.2^{4}}{\epsilon^{4}}<\frac{\delta \eta}{2}$, temos que

$$
\begin{aligned}
P\left\{\left|\beta_{n}(c)\right|>\frac{\epsilon}{2}\right\} & \stackrel{n \rightarrow \infty}{\longrightarrow} P\left\{|N|>\frac{1}{\sqrt{F(c)(1-F(c))}} \frac{\epsilon}{2}\right\} \\
& \leq \frac{\{F(c)(1-F(c))\}^{2} E|N|^{4}}{\left(\frac{\epsilon}{2}\right)^{4}} \\
& \leq \frac{F(c)^{2} \cdot 3 \cdot 2^{4}}{\epsilon^{4}} \\
& \leq \frac{3.2^{4} \cdot \delta^{2}}{\epsilon^{4}} \\
& <\frac{\delta \eta}{2}
\end{aligned}
$$

Assim, para essa escolha adequada de $\delta$ e $n$ excedendo algum $n_{\delta}$, obtemos que

$$
P\left\{\left|\beta_{n}(c)\right|>\frac{\epsilon}{2}\right\}<\frac{\delta \eta}{2}
$$

e, portanto,

$$
P\left\{\sup _{0 \leq s \leq \delta}\left|\beta_{n}(s)\right|>\frac{\epsilon}{2}\right\}<\frac{\delta \eta}{2}
$$

Agora, como

$$
P\left\{\sup _{0 \leq s \leq \delta}\left|\beta_{n}(0)\right|>\frac{\epsilon}{2}\right\}=P\left\{\left|\beta_{n}(0)\right|>\frac{\epsilon}{2}\right\},
$$

o mesmo argumento usado acima para $c=0$ mostra que

$$
P\left\{\sup _{0 \leq s \leq \delta}\left|\beta_{n}(0)\right|>\frac{\epsilon}{2}\right\}<\frac{\delta \eta}{2}
$$

e, assim, por (3.3), (3.4) e (3.5), obtemos

$$
P\left\{\sup _{0 \leq s \leq \delta}\left|\beta_{n}(s)-\beta_{n}(0)\right|>\epsilon\right\}<\frac{\delta \eta}{2}+\frac{\delta \eta}{2}=\delta \eta .
$$

Logo, pelo Teorema 1.9, $\left\{\beta_{n}\right\}$ é rígida. 
Assim, pelos Teoremas 3.1 e 3.2, concluímos, pelo Teorema 1.8, que

$$
\beta_{n}(x) \Rightarrow B(F(x))
$$

\subsection{Convergência Fraca do Processo Quantil Empírico $q_{n}(t)$}

\subsubsection{Convergência de $q_{n}(t)$ para $t$ fixado}

Nesta subseção, provaremos a convergência do processo quantil empírico $q_{n}(t)$, para $t$ fixado. Como já ressaltamos, há uma dificuldade intrínseca na tentativa de tratar o processo quantil empírico com a mesma metodologia adotada para o caso empírico. Assim, algum método alternativo é necessário para eliminar esse problema. Dessa maneira, através do Teorema de Skorohod e um lema de Vervaat adaptado, conseguimos demonstrar a convergência em distribuição, para $t$ fixado, do processo quantil empírico associado a uma cadeia de Markov com espaço de estados geral e ergódica , para a variável aleatória $B_{t}=\frac{B(t)}{f\left(F^{-}(t)\right)}$, utilizando os resultados obtidos para o processo empírico e sua relação com o caso uniforme.

Com base na relação (1.23), o Lema 3.2 fornece o seguinte resultado.

Teorema 3.3 Seja $\left\{X_{n}\right\}_{n \geq 0}$ uma cadeia de Markov com espaço de estados geral e ergódica. Para $F$ contínua e $x$ fixado, se $t=F(x) \in \mathbb{R}$ é tal que $0<F(x)<1$, então

$$
u_{n}(t) \stackrel{d}{\longrightarrow} \mathcal{N}(0, t(1-t)) \stackrel{d}{=} B(t) .
$$

Demonstração. Como $F$ é contínua, basta usarmos a relação $\beta_{n}(x)=u_{n}(F(x))$, fazermos $F(x)=t$ e aplicarmos o Lema 3.2.

Agora, usaremos o Lema 1.5 e o Teorema 1.11 para provarmos a convergência do processo quantil empírico uniforme $v_{n}(t)$, para $t$ fixado.

Teorema 3.4 Seja $\left\{X_{n}\right\}_{n \geq 0}$ uma cadeia de Markov com espaço de estados geral e ergódica. Para $F$ contínua e $x$ fixado, se $t=F(x) \in \mathbb{R}$ é tal que $0<F(x)<1$, então

$$
v_{n}(t) \stackrel{d}{\longrightarrow} B(t) .
$$

Demonstração. Pelo Teorema 3.3, temos que

$$
\sqrt{n}\left(U_{n}(t)-t\right)=u_{n}(t) \stackrel{d}{\longrightarrow} B(t),
$$

no espaço de Skorohod $D[0,1]$. Assim, pelo Teorema 1.11, temos que existem variáveis aleatórias $U_{n}^{*}(t) \stackrel{d}{=} U_{n}(t)$ e $B^{*}(t) \stackrel{d}{=} B(t)$ tais que 


$$
\sqrt{n}\left(U_{n}^{*}(t)-t\right):=u_{n}^{*}(t) \stackrel{q . c .}{\longrightarrow} B^{*}(t),
$$

na topologia Skorohod. Como $B^{*}(t)$ é contínua, temos, pela Observação 1.1, que essa convergência é localmente uniforme, ou seja,

$$
\lim _{n \rightarrow \infty} \sup _{0 \leq t \leq 1}\left|\sqrt{n}\left(U_{n}^{*}(t)-t\right)-B^{*}(t)\right|=0, \text { q.c. . }
$$

Agora, pelo Lema 1.5, temos que

$$
\lim _{n \longrightarrow \infty} \sup _{0 \leq t \leq 1}\left|\sqrt{n}\left(\left(U_{n}^{*}\right)^{-}(t)-t\right)+B^{*}(t)\right|=0 \text {, q.c. . }
$$

Novamente pela equivalência citada na Observação 1.1, temos que a convergência acima ocorre quasecertamente também na topologia Skorohod, isto é:

$$
\sqrt{n}\left(\left(U_{n}^{*}\right)^{-}(t)-t\right) \stackrel{q \cdot c .}{\longrightarrow}-B^{*}(t),
$$

na topologia Skorohod. Além disso, como $\left(U_{n}{ }^{*}\right)^{-}(t) \stackrel{d}{=} U_{n}{ }^{-}(t)$ e $-B^{*}(t) \stackrel{d}{=}-B(t)$, concluímos que

$$
v_{n}(t):=\sqrt{n}\left(U_{n}{ }^{-}(t)-t\right) \stackrel{d}{\longrightarrow}-B(t) \stackrel{d}{=} B(t) .
$$

A seguir, demonstramos a convergência em distribuição, para $t$ fixado, do processo quantil empírico $q_{n}(t)$, na versão não necessariamente uniforme.

Teorema 3.5 Seja $\left\{X_{n}\right\}_{n \geq 0}$ uma cadeia de Markov com espaço de estados geral e ergódica. Se $F$ é absolutamente contínua com densidade $f, x$ está fixado e $t=F(x) \in \mathbb{R}$ é tal que $0<F(x)<1$, então

$$
q_{n}(t) \stackrel{d}{\longrightarrow} \frac{B(t)}{f\left(F^{-1}(t)\right)} .
$$

Demonstração. Pelas hipóteses, temos que

$$
\begin{aligned}
\left|\frac{q_{n}(t)}{\left(F^{-}(t)\right)^{\prime}}-v_{n}(t)\right| & =\left|\frac{\sqrt{n}\left(F_{n}{ }^{-}(t)-F^{-}(t)\right)}{\left(F^{-}(t)\right)^{\prime}}-\sqrt{n}\left(U_{n}{ }^{-}(t)-t\right)\right| \\
& =\left|\sqrt{n}\left(U_{n}{ }^{-}(t)-t\right)\left\{\frac{F_{n}{ }^{-}(t)-F^{-}(t)}{\left(U_{n}^{-}(t)-t\right)\left(F^{-}(t)\right)^{\prime}}-1\right\}\right| \\
& =\left|\sqrt{n}\left(U_{n}{ }^{-}(t)-t\right)\left\{\frac{F^{-}\left(U_{n}^{-}(t)\right)-F^{-}(t)}{\left(U_{n}^{-}(t)-t\right)} \frac{1}{\left(F^{-}(t)\right)^{\prime}}-1\right\}\right| \stackrel{p}{\longrightarrow} 0,
\end{aligned}
$$


pois $\sqrt{n}\left(U_{n}{ }^{-}(t)-t\right) \stackrel{d}{\longrightarrow} B(t)$ e $\frac{F^{-}\left(U_{n}^{-}(t)\right)-F^{-}(t)}{\left(U_{n}{ }^{-}(t)-t\right)} \stackrel{n \longrightarrow \infty}{\longrightarrow}\left(F^{-}(t)\right)^{\prime}$, já que $U_{n}^{-}(t) \longrightarrow t$, uniformemente.

Como $\left(F^{-}(t)\right)^{\prime}=\frac{1}{f\left(F^{-}(t)\right)}$, pois $F(x)$ é absolutamente contínua, concluímos que

$$
q_{n}(t) \stackrel{d}{\longrightarrow}\left(F^{-}(t)\right)^{\prime} B(t)=\frac{B(t)}{f\left(F^{-}(t)\right)} .
$$

\subsubsection{Convergência de $q_{n}(t)$ como Processo}

$\mathrm{Na}$ última subseção, provamos a convergência em distribuição do processo quantil empírico $q_{n}(t)$ para $\frac{B(t)}{f\left(F^{-}(t)\right)}$, com $t$ fixado. A seguir, demonstraremos que a convergência fraca também ocorre no sentido de processos. Como o Lema de Vervaat adaptado 1.5 e o Teorema 1.11 valem para processos gerais, o resultado segue pela convergência fraca já obtida para o caso empírico e os passos da demonstração são análogos à seção anterior.

Com a mesma argumentação usada na demonstração do Teorema 3.3 e pela convergência fraca $\beta_{n}(x) \Rightarrow B(F(x))$, obtemos o seguinte resultado.

Teorema 3.6 Seja $\left\{X_{n}\right\}_{n \geq 0}$ uma cadeia de Markov com espaço de estados geral e ergódica. Para $F$ contínua, se $t=F(x) \in \mathbb{R}$ é tal que $0<F(x)<1$, então

$$
u_{n}(t) \Rightarrow B(t)
$$

Agora, usando o Lema de Vervaat adaptado 1.5 e o Teorema 1.11 no sentido de processos, obtemos o teorema abaixo.

Teorema 3.7 Seja $\left\{X_{n}\right\}_{n \geq 0}$ uma cadeia de Markov com espaço de estados geral e ergódica. Para F contínua, se $t=F(x) \in \mathbb{R}$ é tal que $0<F(x)<1$, então ocorre a convergência fraca

$$
v_{n}(t) \Rightarrow-B(t) \stackrel{d}{=} B(t)
$$

Demonstração. Já obtivemos que $u_{n}(t)$ converge como processo para a Ponte Browniana $B(t)$, isto é,

$$
u_{n}(t):=\sqrt{n}\left(U_{n}(t)-t\right) \Rightarrow B(t),
$$

em $D[0,1]$. Pelo Teorema de Skorohod, existem $u_{n}{ }^{*}(t) \stackrel{d}{=} u_{n}(t)$ e $B^{*}(t) \stackrel{d}{=} B(t)$, definidos em $[0,1]$, tais que

$$
u_{n}^{*}(t) \stackrel{q . c .}{\longrightarrow} B^{*}(t),
$$


em $D[0,1]$. Defina, agora,

$$
U_{n}^{*}(t):=\frac{u_{n}^{*}(t)}{\sqrt{n}}+t
$$

Então, $U_{n}{ }^{*}(t)$ é quase-certamente não-decrescente, pois $U_{n}{ }^{*}(t) \stackrel{d}{=} U_{n}(t)$. Como

$$
\sqrt{n}\left(U_{n}^{*}(t)-t\right):=u_{n}^{*}(t) \stackrel{\text { q.c. }}{\longrightarrow} B^{*}(t),
$$

em $D[0,1]$, e $B^{*}(t)$ tem trajetórias quase-certamente contínuas, essa convergência ocorre localmente uniformemente. Logo, pelo Lema de Vervaat, temos que

$$
\sqrt{n}\left(\left(U_{n}^{*}\right)^{-}(t)-t\right) \stackrel{q . c .}{\longrightarrow}-B^{*}(t),
$$

localmente uniformemente. Ou seja, na topologia Skorohod, em $D[0,1]$,

$$
\sqrt{n}\left(\left(U_{n}^{*}\right)^{-}(t)-t\right) \stackrel{q . c .}{\longrightarrow}-B^{*}(t),
$$

e, como $\left(U_{n}{ }^{*}\right)^{-}(t) \stackrel{d}{=} U_{n}{ }^{-}(t)$, concluímos a convergência fraca

$$
v_{n}(t):=\sqrt{n}\left(U_{n}{ }^{-}(t)-t\right) \Rightarrow-B(t) \stackrel{d}{=} B(t),
$$

como queríamos.

Finalmente, obtemos a convergência fraca do processo quantil empírico $q_{n}(t)$, na versão não necessariamente uniforme.

Teorema 3.8 Seja $\left\{X_{n}\right\}_{n \geq 0}$ uma cadeia de Markov com espaço de estados geral e ergódica. Se F é absolutamente contínua com derivada contínua, densidade $f$ e $0<F(x)<1$, então ocorre a convergência, como processo,

$$
q_{n}(t) \Rightarrow \frac{B(t)}{f\left(F^{-1}(t)\right)}
$$

Demonstração. Defina

$$
\Phi(s)=s .\left(F^{-}(t)\right)^{\prime} .
$$

Como $t \wedge U_{n}{ }^{-}(t) \leq t \leq t \vee U_{n}{ }^{-}(t)$, a equação (1.25) nos dá

$$
q_{n}(t)=\sqrt{n}\left(U_{n}^{-}(t)-t\right) \cdot\left(F^{-}(t)\right)^{\prime}=\left(F^{-}(t)\right)^{\prime} v_{n}(t) .
$$

Assim, como $\Phi(s)$ é contínua, o Teorema 1.7 e (3.6) implicam que

$$
q_{n}(t)=\left(F^{-}(t)\right)^{\prime} v_{n}(t)=\Phi\left(v_{n}(t)\right) \Rightarrow \Phi(B(t))=\left(F^{-}(t)\right)^{\prime} B(t) .
$$

Portanto, concluímos a convergência fraca

$$
q_{n}(t) \Rightarrow \frac{B(t)}{f\left(F^{-}(t)\right)} .
$$




\section{Referências Bibliográficas}

[1] BARBOSA, E. G.; DOREA, C. C. Y. A note on the Lindeberg condition for convergence to stable laws in Mallows distance. Bernoulli 15 922-924, 2009.

[2] BICKEL, P. J.; FREEDMAN, D. A Some Asymptotic Theory for the Bootstrap. The Annals of Statistics, Vol. 9, pp. 1196-1217, 1981.

[3] BILlingSLEY, P. Convergence of Probability Measures. John Wiley \& Sons, New York, 1968.

[4] BREIMAN, L. Probability. Philadelphia, Classics in Applied Mathematics - 7, 1992.

[5] BURKHOLDER, D.L. Distribution Function Inequalities for Martingales. The Annals of Probability, 1(1): 19-42, 1973.

[6] CHUNG, K. L. Markov Chains with Stationary Transition Probabilities. Springer, 1960.

[7] ÇINLAR, E. Introduction to Stochastic Processes, 1975.

[8] CSÖRGO, M.; RÉVÉSZ, P. Strong Approximations in Probability and Statistics. Academic Press, New York, 1981.

[9] DOOB, J.L. Stochastic Processes. Willey Classics Library, 1990.

[10] DOREA, C. C. Y.; FERREIRA, D. B. Conditions for Equivalence Between Mallows Distance and Convergence to Stable Laws. Acta Mathematica Hungarica, Vol. 134(1-2), pp. 1-11, 2012.

[11] DOREA, C.C.Y.; DAVID, H.T; WERNER, W.M. Uniforme $\epsilon$-Independence and the Convergence in Distributon of Randomly Indexed Sequence. Math. Proc., Cambridge Philosofical Society, vol.96, 533-542, 1984.

[12] FELLER, W. An Introduction to Probability Theory and its Applications. Vol. II. Second Edition. New York, John Wiley \& Sons, 1971.

[13] HAAN, L.; FERREIRA, Ana. Extreme Value Theory: An Introduction. Springer, 2006.

[14] HALL, P.; HEYDE, C. C. Martingale Limit Theory and its Application. Academic. New York, 1980. 
[15] JOHNSON, O. ; SAMWORTH, R. Central Limit Theorem and Convergence to Stable Laws in Mallows Distance. Bernoulli, Vol. 11, pp. 829-845, 2005.

[16] KARLIN, Samuel; TAYLOR, Howard M. A First Course in Stochastic Processes. 2a edição. Academic Press, 1975.

[17] MALLOWS, C. L. A Note on asymptotic joint normality. Annals of Mathematical Statistics, Vol. 43(2), pp. 508-515, 1972.

[18] MEYN, S.P.; TWEEDIE, R.L. Markov Chains and Stochastic Stability. New York, 2009.

[19] RESNICK, Sidney I. Heavy-Tail Phenomena. Springer, 2007.

[20] SAMORODNITSKY, G.; TAQQU, M.S. Stable non-Gaussian Random Processes. Chapman and Hall, 1994.

[21] SOARES, Wembesom M. Convergênca em Distância Mallows Ponderada com Aplicações em Somas Parcias e Processos Empíricos. Universidade de Brasília, 2015.

[22] SHORACK, G; WELLNER, J. Empirical Processes with Applications to Statistics. Wiley, New York, 1986.

[23] VERVAAT, Wim. Functional limit theorems for processes with positive drift and their inverses. Z Wahrsch. verw. Gebiete 23, 245-253, 1971.

[24] von Bahr, B.; Esseen, C.G. Inequalities for the rth Absolute Moment of a Sum of Random Variables, The Annals of Mathematical Statistics, 36: 299-303, 1965. 